\title{
GLYCOGEN DISTRIBUTION AND METABOLIC ALTERATIONS \\ IN THE HYPERMUSCULAR, MYOSTATIN MUTANT \\ COMPACT MICE
}

Ph.D. Thesis

Tamás Kocsis MD

Doctoral School of Multidisciplinary Medical Science

Department of Biochemistry

Faculty of Medicine

University of Szeged

Supervisor: Anikó Keller-Pintér, MD, Ph.D.

Szeged

2017 


\section{TABLE OF CONTENTS}

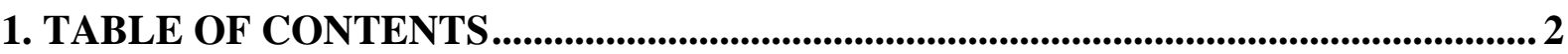

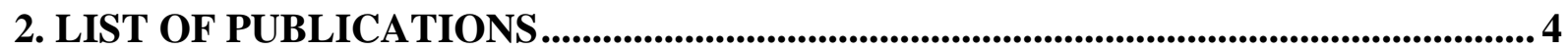

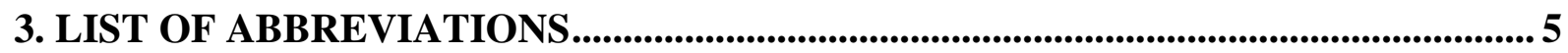

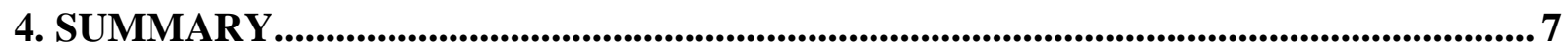

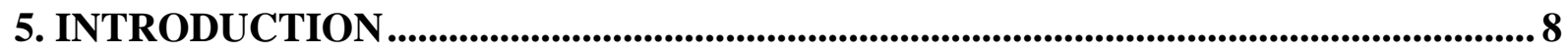

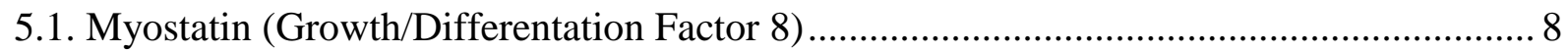

5.2. Myostatin signaling and the regulation of skeletal muscle mass .................................... 9

5.3. Major characteristics of the myostatin knock out mice.................................................. 11

5.4. The role of myostatin in glucose metabolism ......................................................... 12

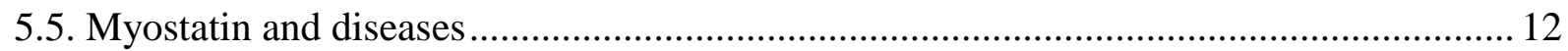

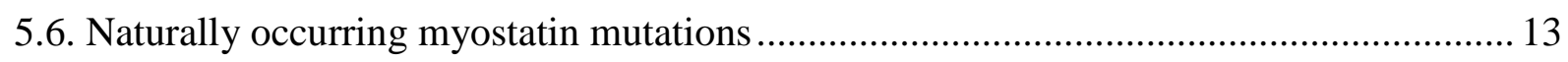

5.7. The Compact mutation and the Compact mice ............................................................ 14

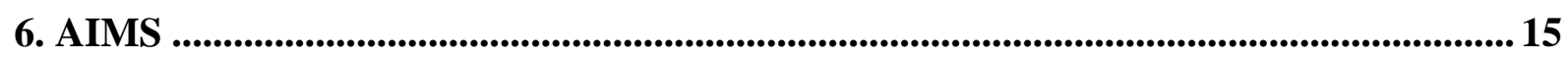

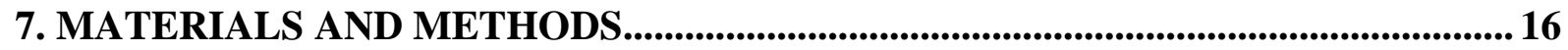

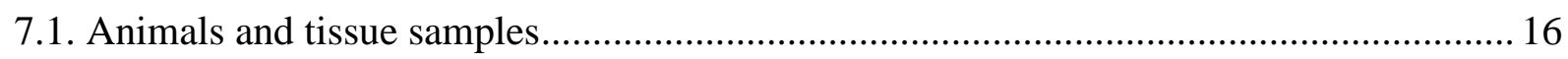

7.2. Determination of glycogen and protein content by spectrophotometry .......................... 16

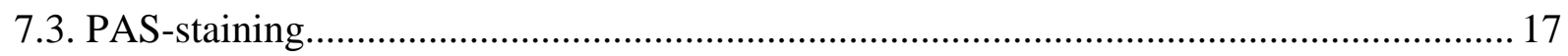

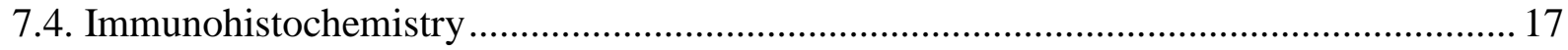

7.5. Determination of the total fiber number and glycogen content of the whole cross sectional

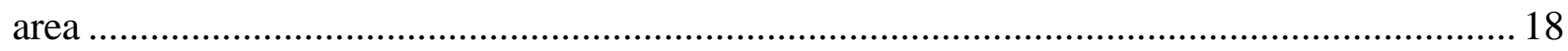

7.6. Determination of the glycogen content of the different fiber types ............................... 19

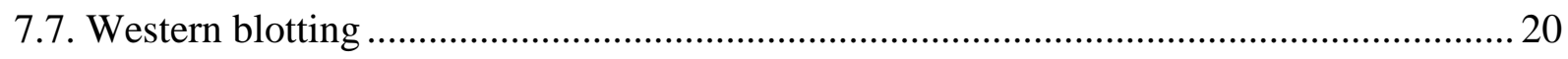

7.8. Measurement of tissue alanine aminotransferase activity ........................................... 21

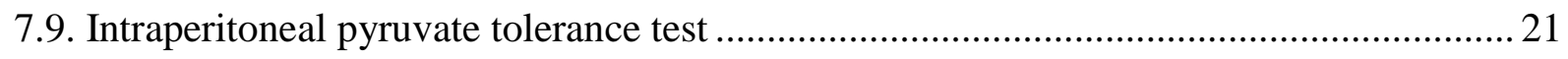

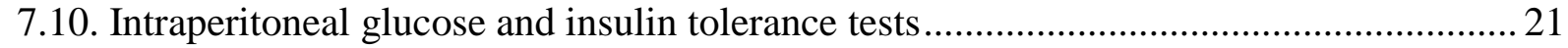

7.11. Small-animal PET/MRI imaging using 2-deoxy-2-( $\left.{ }^{18} \mathrm{~F}\right)$ fluoro-D-glucose ..................... 22

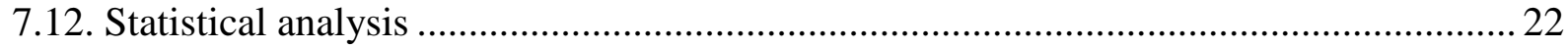




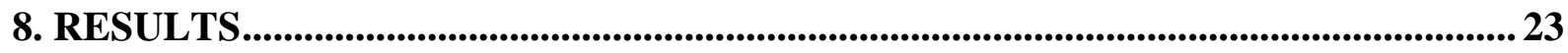

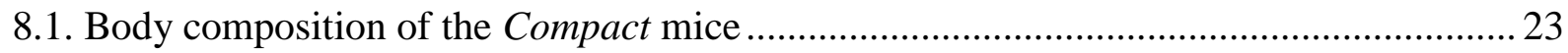

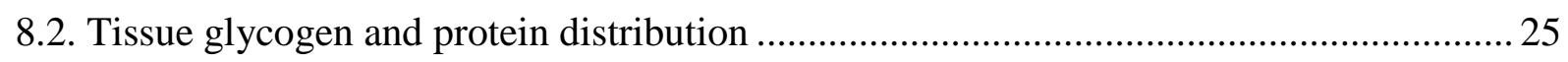

8.3. Skeletal muscle cellularity in Compact mice ............................................................. 27

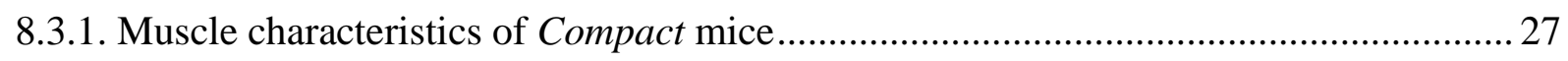

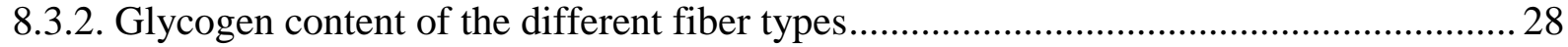

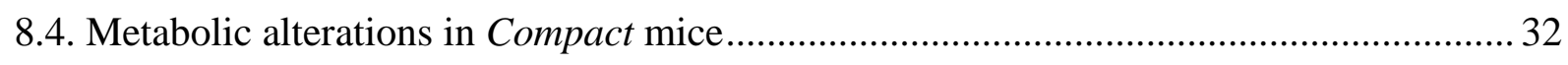

8.4.1. Glucose tolerance and insulin sensitivity are improved by Compact myostatin mutation

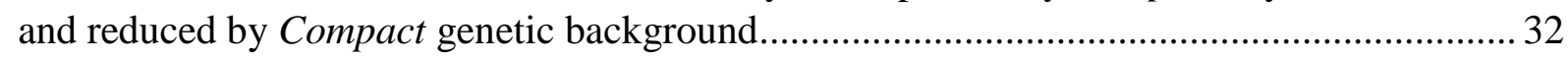

8.4.2. The Compact mutation increases ${ }^{18}$ FDG uptake in skeletal muscle, liver, and adipose

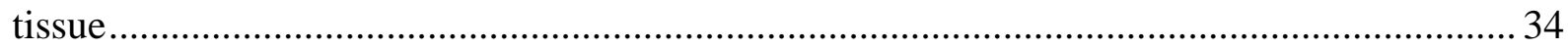

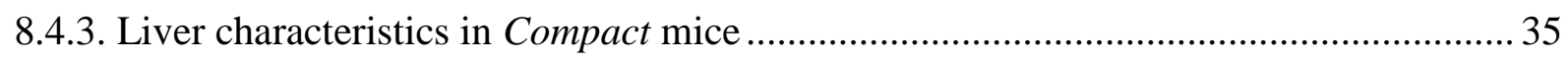

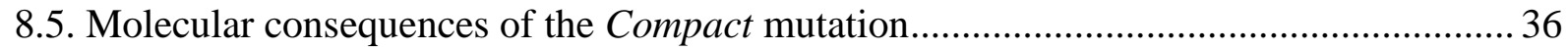

8.5.1. The Compact mutation of myostatin propeptide decreases myostatin formation .......... 36

8.5.2. Effects of Compact myostatin mutation and genetic background on signaling ............. 37

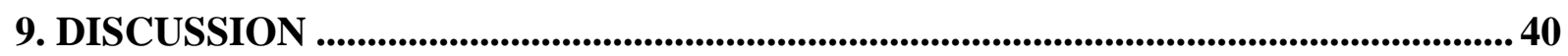

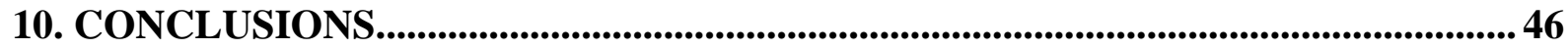

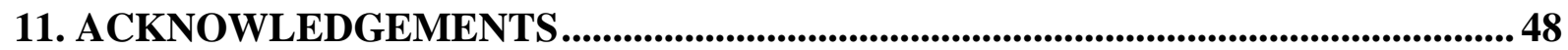

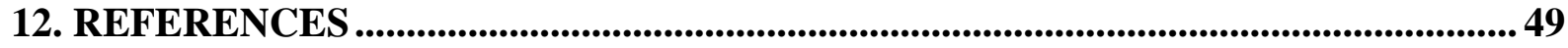

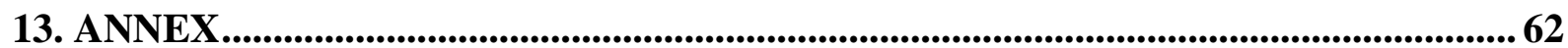




\section{LIST OF PUBLICATIONS}

\section{Publications related to the subject of the dissertation:}

I. Kocsis T, Baán J, Müller G, Mendler L, Dux L, Keller-Pintér A. Skeletal muscle cellularity and glycogen distribution in the hypermuscular Compact mice. Eur J Histochem 58: 2353, 2014, IF: $\mathbf{2 . 0 4 2}$

II. Kocsis T, Trencsenyi G, Szabo K, Baan JA, Muller G, Mendler L, Garai I, Reinauer H, Deak F, Dux L, Keller-Pinter A. Myostatin propeptide mutation of the hypermuscular Compact mice decreases the formation of myostatin and improves insulin sensitivity. Am J Physiol Endocrinol Metab 312(3): E150-E160, 2017, IF: 4.142* (2016)

Cummulative impact factor of papers subjected in the thesis: $6.184^{*}$

2. Other full paper published during the Ph.D fellowship:

Baán JA, Kocsis T, Keller-Pintér A, Müller G, Zádor E, Dux L, Mendler L. The Compact mutation of myostatin causes a glycolytic shift in the phenotype of fast skeletal muscles. J Histochem Cytochem 12: 889-900, 2013, IF: 2.412

Total impact factor: $8.596 *$ 


\section{LIST OF ABBREVIATIONS}

ActIIA and B: Activin receptor type IIA and B

ALT: alanine aminotransferase

ALK: Anaplastic lymphoma kinase

AMPK: AMP activated protein kinase

ANOVA: Analysis of Variance

Arg: arginine

AS160: Akt substrate of $160 \mathrm{kDa}$

AUC: Area Under the Curve

BEHi: Berlin High inbred

BMP: Bone Morphogenic Protein

Cdk: Cycline-dependent kinase

C/EBP $\alpha$ : CCAAT-Enhancer-Binding Proteins $\alpha$

CSA: Cross Sectional Area

DUHi: Dummerstoffer High inbred

EDTA: Ethylenediaminetetraacetic acid

ELISA: Enzyme-linked Immunosorbent Assay

ERK1/2: Extracellular signal-regulated protein kinases 1 and 2

${ }^{18}$ FDG: 2-deoxy-2- $\left({ }^{18} \mathrm{~F}\right)$ fluoro-D-glucose

FSTL-3: Follistatin-related protein 3

FoxO: Forkhead box proteins, subgroup O

FOV: Field of View

GAPDH: Glyceraldehyde-3-Phosphate Dehydrogenase

GASP-1 and GASP-2: GDF-associated serum protein-1 and 2

GAST: $M$. gastrocnemius

GDF-8: Growth/Differentiation Factor 8

GDF-11: Growth/Differentiation Factor 11

GLUT4: Glucose transporter type 4

GS: Glycogen synthase

GSK-3 $\beta$ : Glycogen synthase kinase $3 \beta$ 
HGF: Hepatocyte growth factor

HIV: Human Immunodeficiency Virus

IRS-1: Insulin receptor substrate 1

IGF-1: Insulin-like growth factor 1

KO: Knock out

LAP: Latency Activated Peptide

LTBP-3: Latent-transforming growth factor beta-binding protein 3

MEF2C: Myocyte Enhancer Factor 2C

mstn: myostatin

mTOR: Mammalian target of rapamycin

MuRF-1: Muscle RING-finger protein-1

MyHCI, IIA, IIB, IIX: Myosin Heavy Chain I, IIA, IIB, IIX

NaCl: sodium chloride

OD: Optical Density

PAS: Periodic Acid - Schiff staining

PBS: Phosphate-Buffered Saline

Pcsk6: Protein convertase subtilisin/kexin type 6

PET/MRI: Positron Emission Tomography - Magnetic Resonance Imaging

PGC-1a: Peroxisome proliferator-activated receptor-gamma coactivator-1 alpha

PI3K: Phosphatidylinositol-4,5-bisphosphate 3-kinase

PPAR $\gamma$ : Peroxisome proliferator-activated receptor gamma

PTEN: Phosphatase tensin homologue

QF: M. quadriceps femoris

RSRR: arginine - serine - arginine - arginine sequence

SDS: sodium dodecyl sulfate

SEM: Standard Error of the Mean

SIRT1: sirtuin 1

SUV: Standard Uptake Volume

TA: M. tibialis anterior

TGF- $\beta$ : Transforming Growth Factor $\beta$

VOI: Volume of Interest 


\section{SUMMARY}

The TGF- $\beta$ family member myostatin (Growth/Differentiation factor-8, GDF-8, mstn) is a negative regulator of skeletal muscle growth. The hypermuscular Compact mice carry the 12bp $M \sin (C m p t-d l 1 A b c)$ deletion in the sequence encoding the propeptide region of the precursor promyostatin, and additional modifier genes of the Compact genetic background contribute to determine the full expression of the phenotype. In this study, by using mice strains carrying mutant or wild-type myostatin alleles with Compact genetic background and non-mutant myostatin with wild-type background, we studied separately the effect of the Mstn (Cmpt-dllAbc) mutation or the Compact genetic background on morphology, metabolism, and signaling. Furthermore, we investigated the cellular characteristics and the glycogen distribution of the Compact tibialis anterior (TA) muscle by quantitative histochemistry and spectrophotometry. We show that both the Compact myostatin mutation and Compact genetic background account for determination of skeletal muscle size. Despite the increased musculature of Compacts, the absolute size of heart and kidney is not influenced by myostatin mutation; however, the Compact genetic background increases them. The average glycogen content of the individual muscle fibers kept unchanged, the total amount of glycogen in the Compact TA muscle increased two-fold, which can be explained by the presence of more fibers in Compact compared to wild-type BALB/c muscle. Moreover, the ratio of the most glycolytic IIB fibers significantly increased in the Compact TA muscle, of which glycogen content was the highest among the fast fibers. Both the Compact myostatin and the genetic background exhibit systemic metabolic effects. The Compact mutation decreases adiposity, improves whole body glucose uptake, insulin sensitivity and ${ }^{18}$ FDG (2deoxy-2-[ $\left[{ }^{18}\right.$ F]fluoro-D-glucose) uptake of skeletal muscle and white adipose tissue, whereas the Compact genetic background has the opposite effect. Importantly, the mutation does not prevent the formation of mature myostatin; however, a decrease in myostatin level was observed leading to altered activation of Smad2, Smad1/5/8 and Akt, and increased level of pAS160, a Rab-GTPase activating protein responsible for GLUT4 (Glucose transporter type 4) translocation. Based on our analysis the Compact genetic background strengthens the effect of myostatin mutation on muscle mass, but can compensate for each other when systemic metabolic effects are compared. 


\section{INTRODUCTION}

\subsection{Myostatin (Growth/Differentation Factor 8)}

The TGF- $\beta$ (Transforming Growth Factor- $\beta$ ) superfamily myostain is a potent regulator of the skeletal muscle mass [1]. Myostatin gene knock out in mice causes increased muscle mass with hyperplasia and hypertrophy of the muscle fibers [1]. Furthermore, naturally occurring myostatin gene mutations, e.g., in human [2], mouse [3], cattle [4], or sheep [5] were described resulting in widespread increase of skeletal muscle mass ("doublemuscled" phenotype).

Myostatin regulates the proliferation and differentiation of myoblasts [6, 7]; moreover, it also controls the activation and proliferation of satellite cells, the stem cells of skeletal muscle [8]. However, the effect of myostatin is not restricted to skeletal muscle. Beside the autocrine and paracrine effects, it can serve as an endocrine factor. Myostatin was reported to influence the synthesis and secretion of IGF-1 (Insulin-like Growth Factor-1) in the liver, thereby regulating the amount of circulating IGF-1 [9]. Myostatin expression is limited to a few cell types and tissues $[1,10,11]$. Myostatin is expressed mainly in developing and adult skeletal muscle [1], and is also produced in cardiac muscle [10], mammary gland [12], adipose tissue, or liver [11]. Like other members of the TGF- $\beta$ superfamily, myostatin is synthesized as precursor protein containing a signal sequence, an $\mathrm{N}$-terminal propeptide domain, and a C-terminal domain considered as the active molecule [1] (Figure 1).

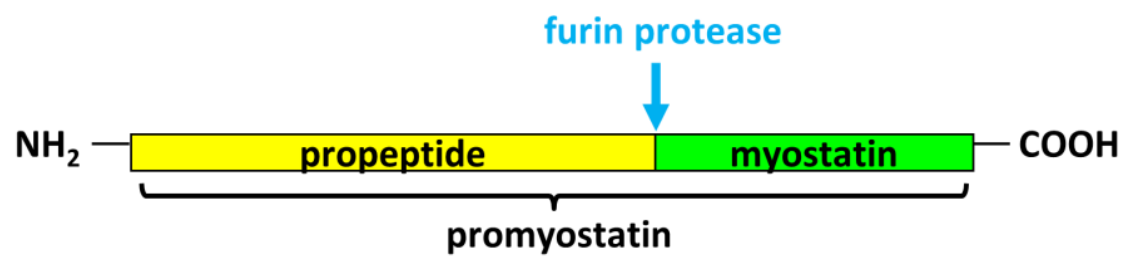

Figure 1. Structure of promyostatin monomer. N-terminal propeptide, C-terminal myostatin domain, and the cleavage site of furin are marked.

The precursor promyostatin undergoes dimerization and proteolytic processing. The promyostatin molecules form a disulfide-linked homodimer following synthesis and translocation into the endoplasmic reticulum $[1,13]$. The promyostatin dimer is cleaved by furin proteases to $\mathrm{N}$-terminal propeptide fragments and $\mathrm{C}$-terminal disulfide-linked myostatin dimer at an Arg-Ser-Arg-Arg site [13, 14, 15]. This cleavage is thought to occur primarily in 
the Golgi apparatus [1, 13]; however, an extracelluar promyostatin pool also exist [16]. Postsecretion activation of this pool by furin proteases may represent a major control point for activation of myostatin in skeletal muscle [16] (Figure 2).

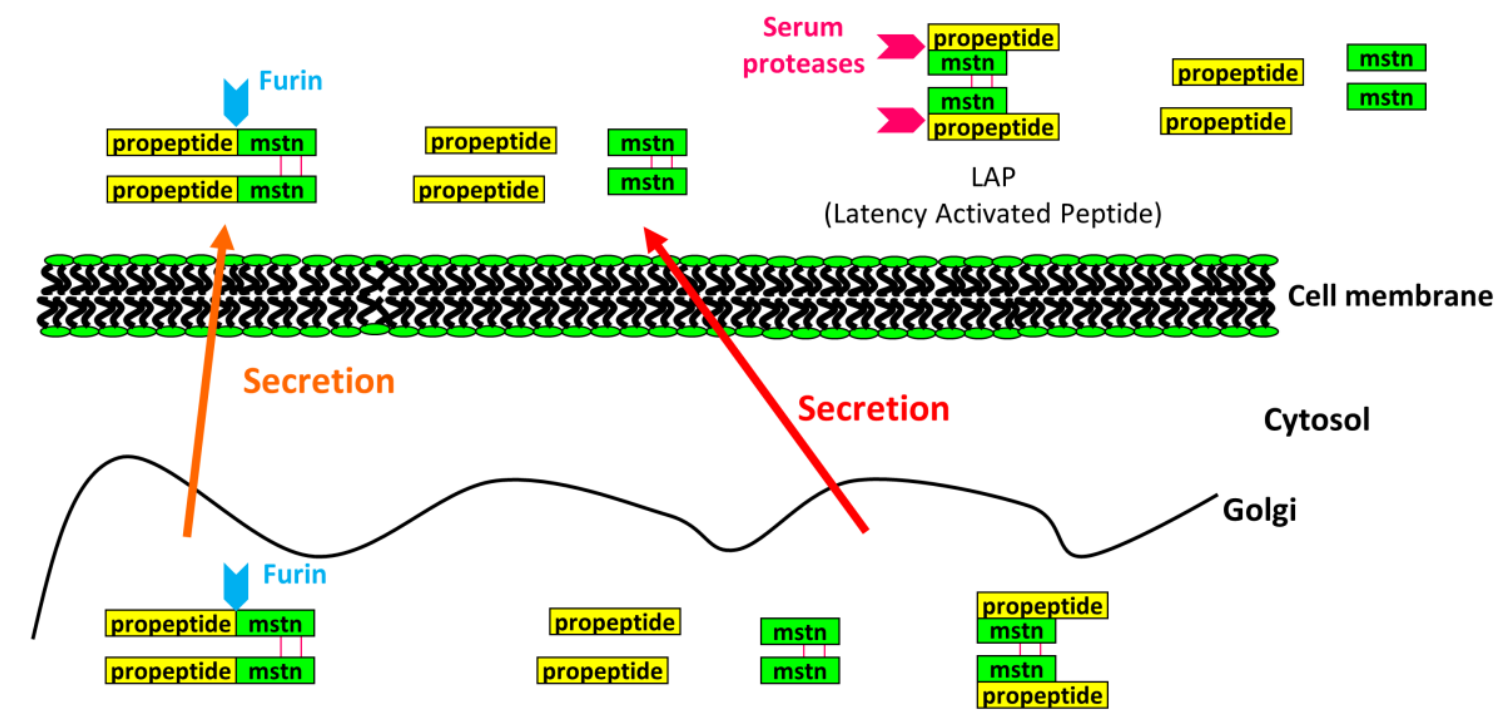

Figure 2. Processing of promyostatin. mstn: myostatin

The propeptides can still associate with myostatin dimer via noncovalent bonds to form a latent complex that sequesters functional myostatin by preventing its binding to the receptor $[13,15]$. The BMP-1/tolloid family of metalloproteinases can activate this latent complex by proteolytic cleavage between Arg-75 and Asp-76 of the myostatin prodomain [17] .

\subsection{Myostatin signaling and the regulation of skeletal muscle mass}

TGF-superfamily members signal through heteromeric receptor complexes composed of two homodimers each of type I and type II serine/threonine kinase receptors [18]. The activated anaplastic lymphoma kinase type I receptor (ALK4 or ALK5) then propagates the signal by phosphorylating Smad2/3 transcriptional factors (Figure 3) [6, 19]. The phosphoSmad2/3 molecules forms an oligomer with Smad4 that translocates into the nucleus where it interacts with Smad binding partners to regulate transcription [20, 21]. Beyond the Smad2/3 mediated signaling, myostatin influences the phosphatidylinositol 3-kinase (PI3K)/Akt pathway, which is the key regulator of the anabolic and catabolic responses in skeletal 
muscle. Myostatin stimulates cyclin D1 degradation though a PI3K/Akt pathway leading to cell cycle arrest [22].

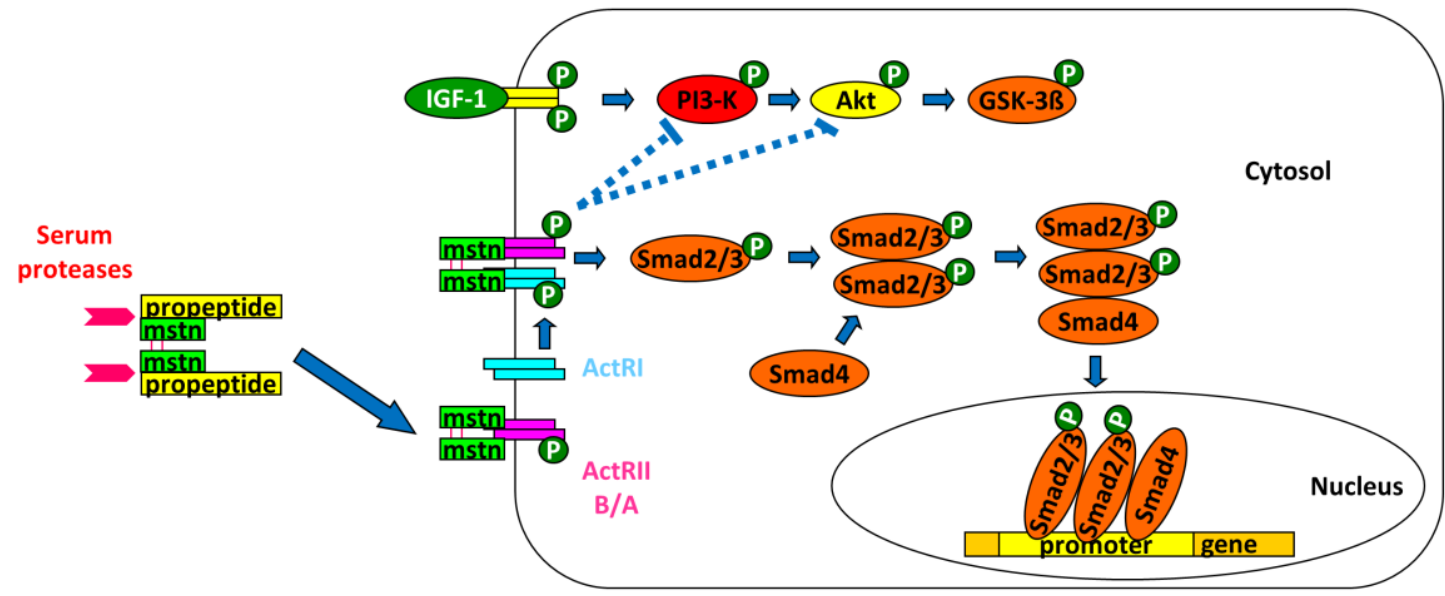

Figure 3. Signal transduction of myostatin. mstn: myostatin; ActRI, ActRII B/A: Activin type receptor I, IIB/A, IGF-1: Insulin-like growth factor 1, PI3-K: Phosphatidil-inositol 3-kinase, GSK-3 $\beta$ : Glycogen synthase kinase $3 \beta$

In addition, myostatin inhibits the activation of Akt/mammalian target of rapamycin $(\mathrm{mTOR}) / \mathrm{p} 70 \mathrm{~S} 6$ protein synthesis pathway, which regulates both differentiation in myoblasts and hypertrophy in myotubes [23].

Activation of FoxO3 (Forkhead box proteins, subgroup $\mathrm{O}$ ) results in the induction of the muscle-specific E3-ubiquitin ligases atrogin-1 and muscle RING-finger protein-1 (MuRF1) $[24,25]$. In muscle, FoxOs are known to interact with Smad3 and Smad4 inducing protein degradation. It was shown that FoxO1 and Smad synergistically increase the expression of myostatin mRNA, and its promoter activity in $\mathrm{C} 2 \mathrm{C} 12$ myotubes but via different pathways [26]. Myostatin has also been reported to downregulate the expression of MyoD consequently inhibiting the proliferation and differentiation of the myoblasts [6]. Furthermore, myostatin upregulates p21, a Cdk (Cycline-dependent kinase) inhibitor, and decreases the levels and activity of Cdk2 protein in satellite cells. Therefore, myostatin negatively regulates the $\mathrm{G}_{1}$ to $\mathrm{S}$ progression and maintains the quiescent status of satellite cells; therefore, negatively regulating satellite cell self-renewal [8].

The BMP (Bone morphogenic protein) pathway is a positive regulator of muscle mass. Increasing the expression of BMP7 or the activity of BMP receptors in muscles induced 
hypertrophy that was dependent on Smad1/5-mediated activation of mTOR signaling [27]. Furthermore, the Smad4, a shared element of the myostatin and BMP pathways, is required to maintain muscle mass and prevent muscle wasting [28].

Multiple extracellular inhibitors limit access of myostatin to cell surface receptors by binding mature myostatin: follistatin [13]; FSTL-3 (Follistatin-related protein 3) [29]; GASP-1 (GDFassociated serum protein-1) [30], and GASP-2 [31]. The follistatin, FSTL-3, and GASP-1/2 act to prevent the receptor binding of circulating mature myostatin. Decorin, a matrix associated small proteoglycan, also binds myostatin which prevents the interaction of myostatin with its receptor [32]. In additional, coexpression of LTBP-3 (Latent-transforming growth factor beta-binding protein 3) with myostatin dcreases phosphorylation of Smad2, and ectopic expression of LTBP-3 in mature mouse skeletal muscle increases fiber area, consistent with reduction of myostatin activity [16].

\subsection{Major characteristics of the myostatin knock out mice}

The myostatin null animals are significantly larger than wild-type mice and exhibit a large and widespread increase in skeletal muscle mass [1]. Individual muscles of mutant animals weight 2-3 times more than those of wild-type animals, and the increase in mass is the result of a combination of muscle cell hyperplasia and hypertrophy [1]. Detailed histological analysis showed that the myostatin KO (knock out) mice exhibit increased ratio of fast glycolytic, type IIB fibers with a concomitant decrease in oxidative type IIA and type I fibers in tibialis anterior and biceps femoris muscles [33]. Notably, this increased muscle mass is not accompanied by the proportionate increase in specific muscle force [34].

Myostatin gene knock out causes a switch between myogenesis and adipogenesis; the myostatin knock out mice exhibit decraesed fat depot and lower serum leptin contentration and mRNA levels in adipose tissue. CCAAT/enhancer binding protein-(C/EBP) and peroxisome proliferator-activated receptor-(PPAR) levels in adipose tissue were significantly lower in myostatin knock out mice compared to wild type mice [35].

Furthermore, the myostatin -/- mice exhibit setting of normal food intake (relative to body weight), normal body temperature, and a slightly decreased resting metabolic rate [36].

Beyond the regulation of muscularity, myostatin was shown to influence the size of internal organs. Knocking out of myostatin resulted in decreased weight of the liver and 
kidney as proportional to body weight $[37,38]$. The ActRIIB receptor (activin receptor type IIA) is expressed in the mouse liver suggesting that the liver is a direct target of the myostatin [39].

\subsection{The role of myostatin in glucose metabolism}

Several studies suggest that loss of myostatin or reduction in active myostatin levels leads to increased insulin sensitivity. Myostatin-null mice exhibit increased insulin sensitivity [40], which depends on AMP-activated protein kinase [41]. In accordance with increased insulin sensitivity, elevated levels of GLUT4 (Glucose transporter type 4), p-Akt and insulin receptor substrate-1 were detected in myostatin -/- muscles; furthermore, increased expression and activity of AMPK and AMPK downstream target genes, Sirt1 and Pgc-1 $\alpha$ in skeletal muscle were also observed [41]. Notably, the senescent myostatin knock out mice exhibit lower serum insulin and glucose levels compared to controls indicating the age-related changes of the insulin resistance [42]. However, myostatin was reported to affect glucose metabolism; the fed and fasting glucose levels of myostatin knock out male mice were unchanged between the mutant and control animals [36].

Transgenic expression of myostatin propeptide prevents diet-induced obesity and insulin resistance [43], and the overexpression of follistatin-like 3, an inhibitor of members of the TGF $\beta$ family [44], or inhibition of myostatin by dominant-negative myostatin receptor [45] also improves insulin sensitivity. Myostatin treatment was found to reduce the glycogen content of C2C12 myoblasts [46].

\subsection{Myostatin and diseases}

Increased serum myostatin levels were measured in several muscle-wasting diseases e.g. sarcopenia [47], chronic obstructive pulmonary disease [48], HIV (Human Immunodeficiency Virus)-infected patients [49], and chronic kidney disease [50]. Beside the increased serum myostatin levels an increase of the intramuscular myostatin level was detected in HIV-infected patients [49] and patients with cancer cachexia [51]. Furthermore, the myostatin mRNA levels were elevated in chronic disuse wasted muscle [52].

Patients with heart failure frequently present skeletal muscle atrophy, reductions muscle strength, muscle size, strength per unit muscle [53, 54]. In additional, higher 
myostatin serum levels were measured in patients with heart failure [55]. Furthermore, more myostatin propeptide was detected by Western blot in the myocardium of patients with

dilatative cardiomyopathy and ischaemic cardiomyopathy, despite the levels of the promyostatin remained unchanged [56].

Obesity is associated with endocrine abnormalities that predict the progression of insulin resistance to type 2 diabetes. Therefore, increased plasma and muscle promyostatin and myostatin levels were described by Western blot analysis in extremly obes insulinresistant human individuals in vivo and verfied by human cultured myotubes in vitro [57]. Intrestingly, Der-Sheng Han and collegaues described decreased serum myostatin levels by ELISA in patients with metabolic syndrome but the ELISA (Enzyme-linked Immunosorbent Assay) kit they employed detected the promyostatin, propeptide and myostatin [58].

Blockade of endogenous myostatin by using intraperitoneal injections of blocking antibodies for three months resulted in an increase in body weight, muscle mass, muscle size and absolute muscle strength in $m d x$ mouse (mouse model for Duchenne and Becker muscular dystrophy) muscle along with a significant decrease in muscle degeneration and concentrations of serum creatine kinase [59]. Given these preclinical results, myostatin has been considered a therapeutic target for the treatment of muscular dystrophy (Becker muscular dystrophy, facioscapulohumeral dystrophy and limb-girdle muscular dystrophy. MYO-029 is a recombinant human antibody that binds with a high affinity to myostatin and inhibits its activity. After the phase I/II trials by the MYO-029 the morphometric analysis showed a dose-dependent increase in fiber size diameter [60].

\subsection{Naturally occurring myostatin mutations}

Several naturally occurring mutations of the myostatin gene were reported in various species resulting hypermuscularity ("double muscled" phenotype). Natural mutations of the cattle myostatin can affect the bioactive domain of myostatin: the 11-nucleotide deletion in Belgian Blue (nt821(del11)) causes a premature stop codon in the myostatin transcript eliminating virtually all of the mature, active region of the molecule; whereas, Piedmontese cattle present a G938A transition resulting in a full-length; however, a misfolded, and dysfunctional myostatin [4, 61]. Mutations in the propeptide leading an early STOP codon (nt419(del7ins10), Q204X, and E226X) have also been identified [62]. 
Myostatin mutations were also reported in other species e.g. dogs [63], sheep [64] resulting widespread increase of the muscle mass. Furthermore, myostatin gene mutation was reported in a child; a guanine-adenine transition resulted in a premature STOP codon in the human myostatin transcript leading to a truncated, dysfunctional myostatin [2].

\subsection{The Compact mutation and the Compact mice}

The naturally occurring Compact mutation of the myostatin gene arose in a selection program on high protein amount and body weight conducted at the Technical University of Berlin [65, 66]. Genetic analysis of the Hungarian subpopulation of the hypermuscular Compact mice identified a 12-bp deletion, denoted Mstn $(\mathrm{Cmpt}-\mathrm{dllAbc})$, in the propeptide of the promyostatin [67]. This non-frameshift mutation leads to the shortening of the propeptide region (Figure 4). Both the furin cleavage site and the biologically active growth factor domain of myostatin are unaffected by the Compact mutation. Therefore, the loss of myostatin activity cannot be explained by disruption of the growth factor bioactive domain; however, the mutation can lead to misfolding, or defect in secretion and mistargeting of mature myostatin [67]. Additional modifier genes should be present to determine the full expression of the Compact phenotype; however, these modifier genes of the special Compact genetic background have not yet been identified [68, 69].
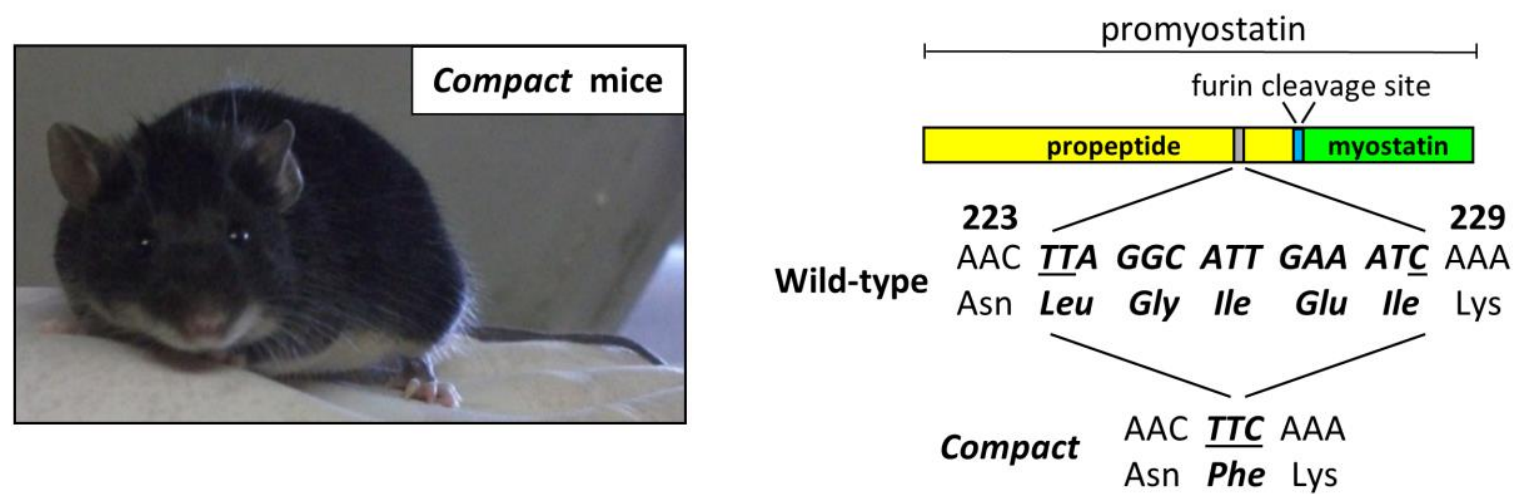

Figure 4. The phenotype of the Compact mice, and the natural mutation in the propeptide region of the myostatin precursor.

A: adenine, $C$ : cytosine, $T$ : timine, $G$ : guanine, Glu: glutamate, Ser: serine, Asn: asparagine; Leu: leucine, Gly: glycine, Ile: isoleucine, Lys: lysine, Ala: alanine, Phe: phenylalanine

Markers on several chromosomes (chromosomes 1, 3, 5, 7, 11, 16 and X) showed linkage with the putative modifiers, and the strongest association was found for markers on 
chromosomes 16 and X [68, 69]. Myogenin is a candidate on the chromosome 1 [69], which has been reported as a downstream target of myostatin [6]. The candidates are MyoD1, a downstream effector of the myostatin [6] and Pcsk6 (Protein convertase subtilisin/kexin type $6)$, involved in proteolytic processing of TGF- $\beta$ members. Further candidates are the chordin on chromosome 16, which binds BMPs and sequesters them in a latent complex [70] and the androgen receptor on the chromosome $\mathrm{X}$ [68].

The Compact mice are hypermuscular and the number of muscle fibers increased (hyperplasia) despite the area of the muscle fibers remain unchanged [71, 72]. The proportion of the fast, glycolytic fibers (IIB, IIX) increased in M. rectus femoris [71] and in M. tibialis anterior, M. extensor digitorum longus [72]. The capillary number per fiber is decreased in the fast, slow and hybrid muscles while the proportion of the area/capillary number is increased [71]. Furthermore, decreased specific force [73] and reduced calcium release from sarcoplasmic reticulum [74] were reported in Compact muscles.

\section{AIMS}

Although the Compact mutation was identified in 1998 [3], its precise molecular effects have not yet been examined. In this study, by using a congenic wild-type mice strain with wild-type myostatin and Compact genetic background, we could separately study the effect of Compact myostatin mutation and genetic background on morphology, metabolism, and signaling.

We defined the following aims in our study:

1. To analyse the body composition of the Compact mice.

2. To characterize the tissue glycogen distribution and skeletal muscle cellularity in Compact mice.

3. To examine the metabolic effects of the Compact mutation.

4. To identify the molecular consequences of the Compact mutation which can regulate skeletal muscle and liver size, and the metabolism. 


\section{MATERIALS AND METHODS}

\subsection{Animals and tissue samples}

The Compact line carrying the 12-bp deletion in the propeptide of promyostatin was selected and inbred in a long-term selection experiment in Berlin, Germany [66, 75]. The Hungarian subpopulation of the Compact line was inbred and kept in the Institute for Animal Biology, Agricultural and Animal Center (Gödöllö, Hungary) for more than 20 years, and they have been breeding in the Department of Biochemistry, Faculty of General Medicine, University of Szeged (Szeged, Hungary) since 2010. The BALB/c mice carrying wild-type myostatin were obtained from the Biological Research Centre of the Hungarian Academy of Sciences (Szeged, Hungary). The Compact mice were crossed with BALB/c to introgress the wild-type myostatin gene of BALB/c to Compact mice. The wild-type myostatin allele was followed through five generations of repeated backcrossing with the Compact line. Heterozygous animals of this line in generation B5 were mated inter se to give homozygous wild-type animals with Compact genetic background (denoted as congenic wild-type animals). Genotyping for Compact myostatin mutation (Mstn(Cmpt-dl1 Abc) was described earlier [3]. Since the Compact hypermuscular phenotype is stronger in males than females [68], we performed the study on male, 3-4 and 10 months old homozygous mice. Animal experiments conformed to the National Institutes of Health Guide for the Care and Use of Laboratory animals (NIH Pub. No. 85-23, Revised 1996) and were approved by the local Ethics Committee at the University of Szeged.

The animals were kept under controlled temperature with 12/12h light/dark cycles, and were fed standard chow and tap water ad libitum. The mice were anaesthetized by intraperitoneal injection of chloral hydrate (3\% chloral hydrate, $0.15 \mathrm{ml} / 10 \mathrm{~g}$ body weight), and the quadriceps femoris (QF), gastrocnemius (Gastro), tibialis anterior (TA) muscles, and organs were removed. The tissue samples were frozen immediately in isopentane cooled by liquid nitrogen and stored at $-80^{\circ} \mathrm{C}$ until further processing.

\subsection{Determination of glycogen and protein content by spectrophotometry}

Non-fasting 3-4 months old animals were sacrificed to determine tissue glycogen content. Muscle and liver glycogen was measured as glucose residues after acidic hydrolysis by a standard enzymatic assay. Briefly, following cryogenic milling the samples were 
digested for 1.5 hour in $2.0 \mathrm{M} \mathrm{HCl}(250 \mu \mathrm{HCl} / 10 \mathrm{mg}$ muscle and $500 \mu \mathrm{HCl} / 10 \mathrm{mg}$ liver $)$ at $100^{\circ} \mathrm{C}$. After lysis the samples were cooled to room temperature and neutralized by adding of equal amount of $2.0 \mathrm{M} \mathrm{NaOH}$. Thereafter, the samples were centrifuged for $10 \mathrm{~min}$ at $21000 \times \mathrm{g}$ (Hettich Universal 320R, DJB, Labcare Ltd, Buckinghamshire, UK) at room temperature, and the supernatants were removed. The concentration of glucose was determined from the supernatant by Hexokinase kit (Roche, Mannheim, Germany).

Muscles were homogenized in a buffer $(0.1 \mathrm{M}$ Tris-HCl pH 8.0, 0.01 M EDTA, 10\% SDS) containing protease inhibitor cocktail (Sigma-Aldrich, St. Louis, Mo, USA) to extract the total protein amount. The incubation of the samples at $99^{\circ} \mathrm{C}$ for $60 \mathrm{sec}$ was followed by centrifugation at $11000 \times \mathrm{g}$ for $5 \mathrm{~min}$ (Hettich Universal 320R, DJB, Labcare Ltd, Buckinghamshire, UK) at room temperature to remove cellular debris. The protein content of the supernatant was determined by BCA Protein Assay Reagent (Thermo Scientific, Rockford, IL, USA) in agreement with the manufacturer's instructions. Spectrophotometry was performed on Fluostar Optima (BMG Labtech, Ortenberg, Germany) and data were analyzed on Mars Data Analysis Software (BMG Labtech, Ortenberg, Germany).

\subsection{PAS-staining}

Glycogen was detected by performing Periodic Acid Schiff (PAS)-staining on $10 \mu \mathrm{m}$ cryosections of the TA muscle and $5 \mu \mathrm{m}$ sections of the liver samples. Sections were fixed for 1 hour at $4{ }^{\circ} \mathrm{C}$ in $3.7 \%$ formaldehyde in ethanol immediately to avoid glycogen breakdown, and incubated for 15 min with $0.5 \%$ periodic acid (Sigma Aldrich, St. Louis, MO, USA) at room temperature, followed by $5 \mathrm{sec}$ washing in tap water and deionized water four times. Then the sections were incubated in Schiff's reagent for 1 hour at room temperature, followed by 5 min incubation (twice) in potassium metabisulfite in deionized water. Thereafter, gently washing of the slides for $10 \mathrm{sec}$ under running hand-warm tap water was followed by washing three times for $5 \mathrm{sec}$ in deionized water. Finally, the sections were incubated for $30 \mathrm{sec}$ in 50, $70,90,100 \%$ ethanol and for $15 \mathrm{sec}$ in toluol, then were mounted with Entellan.

\subsection{Immunohistochemistry}

Fiber-type analysis was performed on $10 \mu \mathrm{m}$ serial cryosections of the midbelly region of TA muscle. The sections were blocked in 5\% non-fat dry milk (BioRad, Berkeley, 
California, USA) in PBS, and then incubated with mouse monoclonal primary antibodies. BA-D5 (1:25), sc-71 (1:25) and BFF3 (1:5) primary antibodies were used, specific for Myosin Heavy Chain I (MyHCI, slow oxidative), MyHCIIA (fast oxidative) and MyHCIIB (fast glycolytic) [76, 77]. After incubation with the peroxidase-conjugated secondary antibody (rabbit anti-mouse; Dako, Denmark), the immunocomplexes were visualized by 3,3'diaminobenzidine. We could not detect BA-D5 positive (MyHCI) fibers in the TA muscle; therefore, the fibers stained with neither MyHCIIA nor MyHCIIB were considered as MyHCIIX fibers.

\subsection{Determination of the total fiber number and glycogen content of the whole cross} sectional area

Photos were taken with 10x objective using a Nikon Labophot-2 microscope (Nikon Inc., Japan) equipped with Olympus DP71 camera. The full cross sectional areas (CSAs) of the muscles were reconstructed from the microscopic images by Cell*B software (Olympus DP Soft software, Version 3.2., Soft Imaging System GmbH; Munster, Germany) (Figure 5A). The glycogen content of an individual fiber $\left(\mathrm{OD}^{*} \mu \mathrm{m}^{2}\right)$ can be predicted by multiplying the average intensity of PAS-staining by the CSA (cross sectional area) of the fiber.

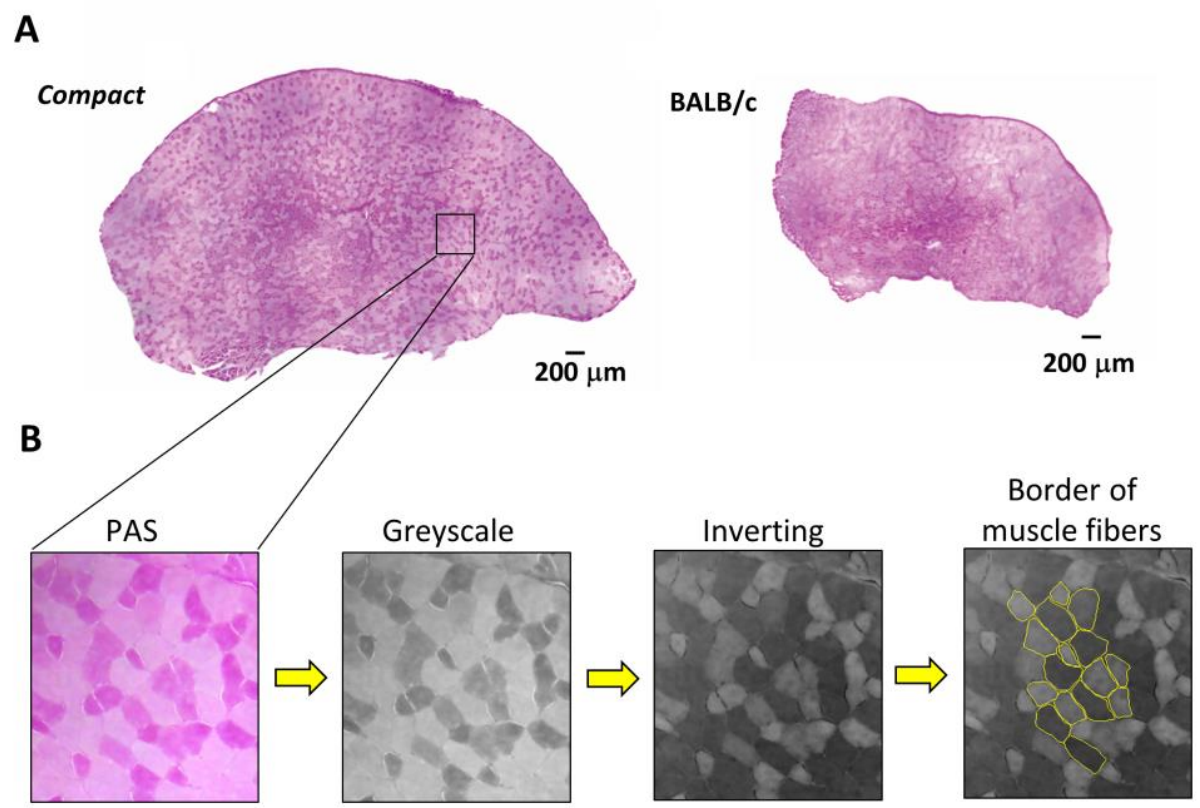

Figure 5. Determination of the average glycogen content of the PAS-stained muscle fibers was performed on panoramic images representing the full cross sectional area of the muscle $(A)$. During the determination the greyscale images of PAS-staining were inverted, and then the borders of the fibres were marked with circles $(B)$ to measure the area of the fibres and the optical density of PAS-staining. PAS: Periodic Acid Schiff-staining. 
Average intensity (optical density, OD) of PAS-staining (0-1 OD), CSAs of all fibers $\left(\mu \mathrm{m}^{2}\right)$ and fiber numbers were determined on the whole muscle CSA on greyscale converted PASstained, panoramic images (Figure 5B) by Digimizer software (Medcalc software, Mariakelke, Belgium).

\subsection{Determination of the glycogen content of the different fiber types}

The fiber-type specific average intensity of PAS-staining and the CSAs of the different fiber types were determined (Figure 6A, B) on 2-3 representative microscopic fields of both superficial and deep regions of the TA muscle (400-1000 fibers/sample, 14-17\% of total fiber number) with 10x objective).

A

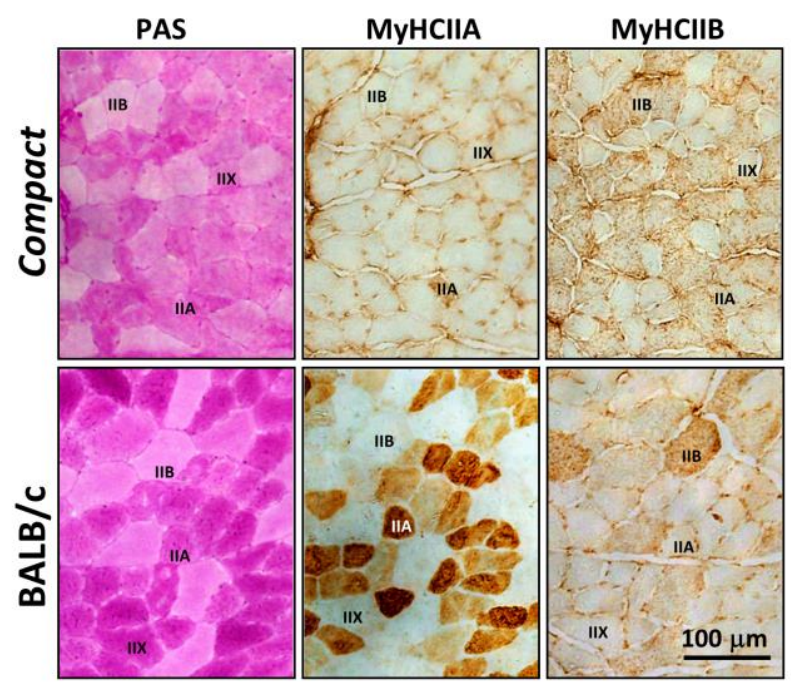

B

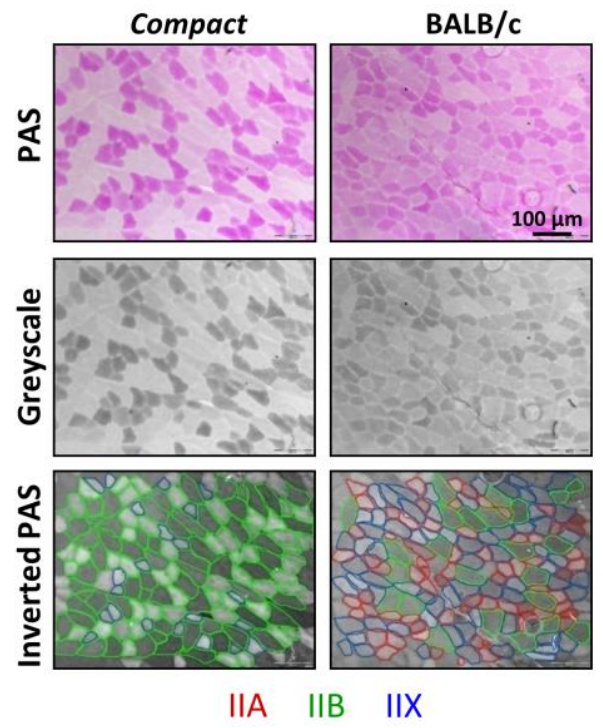

Figure 6. Representative images of the deep region of tibialis anterior muscle. Serial sections were stained by either PAS or antibodies against MyHCIIA and MyHCIIB; representative fibers marked as IIA, IIB, and IIX (A). Both the anti-MyHCIIA and anti-MYHCIIB stained samples were matched with the identical regions of PASstained images to determine the glycogen content of the individual muscle fiber-types (B). PAS: Periodic AcidSchiff staining, MyHCIIA: Myosin Heavy Chain kinase IIA, MyHCIIB: Myosin Heavy Chain kinase IIB.

These muscle regions were selected for analysis because the tibialis anterior muscle shows an increasing gradient of fibres having a high oxidative enzyme activity proceeding from the superficial to the deep region of the muscle [78]. Both the sc-71 (MyHCIIA) and the BF-F3 (MyHCIIB) stained regions were matched with the PAS-stained identical regions on serial sections to analyze the glycogen content of the different fiber types. The glycogen index 
(\%) of a fiber-type was defined as the total glycogen amount of a fiber type / total glycogent content of the muscle.

\subsection{Western blotting}

To analyze protein expression levels M. gastrocnemius and liver samples of 3-4 months old male animals were homogenized at $4^{\circ} \mathrm{C}$ in a buffer $[50 \mathrm{mM}$ Tris- $\mathrm{HCl}(\mathrm{pH}$ 7.6), $100 \mathrm{mM} \mathrm{NaCl}, 10 \mathrm{mM}$ EDTA] supplemented with protease inhibitor cocktail (Sigma-Aldrich, St. Louis, MO, USA), $1 \mathrm{mM}$ natrium-orthovanadate and $1 \mathrm{mM}$ natrium-fluoride. Following centrifugation at $4^{\circ} \mathrm{C}$ for $10 \mathrm{~min}$ at $11000 \times \mathrm{g}$ (Hettich Universal 320R, DJB, Labcare Ltd, Buckinghamshire, UK) to remove cellular debris the protein concentration of the supernatants was determined by BCA kit (Thermo Scientific).

Table 1. Antibodies used for Western blotting

\begin{tabular}{lllc}
\hline \hline Target protein & \multicolumn{1}{c}{ Manufacturer } & \multicolumn{1}{c}{ Species } & Dilution \\
\hline Phospho-Akt & Cer473 & mouse; monoclonal & $1: 1000$ \\
Phospho-Smad1/5/8 ${ }^{\text {Ser463/465 }}$ & Cell Signaling, \#9511 & rabbit; polyclonal & $1: 1000$ \\
Phospho-Smad2 ${ }^{\text {Ser465/Ser467 }}$ & Invitrogen, 44-244G & rabbit; polyclonal & $1: 1000$ \\
Phospho-AS160 ${ }^{\text {Thr642 }}$ & Cell Signaling, \#8881S & rabbit; polyclonal & $1: 1000$ \\
Smad4 & Santa Cruz, sc-7966 & mouse; monoclonal & $1: 5000$ \\
Akt (pan) & Cell Signaling, \#4691 & rabbit; polyclonal & $1: 1000$ \\
Myostatin & Millipore, AB3239-I & rabbit; polyclonal & $1: 1000$ \\
GDF8 propeptide & R\&D, MAB7881 & rat; polyclonal & $1: 500$ \\
GLUT4 & Cell Signaling, \#2213S & mouse; polyclonal & $1: 1000$ \\
GAPDH & Cell Signaling, \#2118 & rabbit; monoclonal & $1: 5000$ \\
HRP-Conjugated Anti-Rabbit IgG & DAKO, P0448 & goat; polyclonal & $1: 2000$ \\
HRP-Conjugated Anti-Mouse IgG & DAKO, P0161 & rabbit; polyclonal & $1: 5000$ \\
HRP-Conjugated Anti-Rat IgG & Jackson, 112-035-003 & goat; polyclonal & $1: 10000$ \\
\hline
\end{tabular}

The samples were separated on $10 \%$ SDS-polyacrylamide gel under reducing or nonreducing conditions, and were transferred onto Protran nitrocellulose membrane (Amersham, GE Healthcare). After incubation in 5\% blocking agent (Biorad, USA) the membrane was incubated with primary antibodies (Table 1), followed by incubation with appropriate horseradish peroxidase-conjugated anti-IgG secondary antibody (Table 1). Mouse 
recombinant myostatin (788-G8, R\&D Systems) was used as a positive control. ECL reagent (Advansta, Menlo Park, CA, USA) was used for substrate detection, and the membrane was exposed to X-ray film (AGFA) for visualization.

\subsection{Measurement of tissue alanine aminotransferase activity}

Liver samples of 3-4 months old male mice were frozen in liquid nitrogen and tissue homogenates were prepared as described at Western blotting. The activity of alanine aminotransferase (ALT) enzyme of the samples was determined by lactate dehydrogenase coupled kinetic colorimetric assay (Diagnosticum Inc., Budapest, Hungary) in accordance with the manufacturer's instructions. Spectrophotometry was performed with Fluostar Optima (BMG Labtech, Ortenberg, Germany).

\subsection{Intraperitoneal pyruvate tolerance test}

3-4 months old male mice were fasted for 16 hours before intraperitoneal pyruvate tolerance test and had free access to water. Following the measurement of baseline blood glucose levels mice were injected with intraperitoneal pyruvate $(2 \mathrm{mg}$ pyruvate / $1 \mathrm{~g}$ body weight). Blood glucose was measured from distal tail vein at 15, 30, 45, 60, 90, and 120 min. All blood glucose measurements were performed by AccuCheck blood glucose monitoring system (Roche, Germany).

\subsection{Intraperitoneal glucose and insulin tolerance tests}

For intraperitoneal glucose tolerance test, 3-4 and 10 months old male mice were fasted for 16 hours and had free access to water. The measurement of baseline blood glucose was followed by intraperitoneal injection of D-glucose ( $2 \mathrm{mg}$ glucose / $1 \mathrm{~g}$ body weight) and blood glucose was determined from distal tail vein at 30, 60, 90, and $120 \mathrm{~min}$.

For intraperitoneal insulin tolerance test, animals in same age were fasted for 5 hours, had free access to water and their baseline blood glucose was measured. After the injection of intraperitoneal insulin bolus (1.0 U / 1 kg body weight; Humulin R, Eli Lilly, B.V Grootslag, Netherlands) blood glucose was measured from distal tail vein at 15, 30, 45, 60, 90, and 120 min. Blood glucose measurements were performed by AccuCheck blood glucose monitoring system (Roche, Germany). 


\subsection{Small-animal PET/MRI imaging using 2-deoxy-2-( ${ }^{18}$ F)fluoro-D-glucose}

10 months old male mice were injected with 7.0 $\pm 0.2 \mathrm{MBq}$ of ${ }^{18}$ FDG (2-deoxy-2$\left({ }^{18} \mathrm{~F}\right)$ fluoro-D-glucose) via the lateral tail vein in $0.2 \mathrm{ml}$ volume. $50 \mathrm{~min}$ after ${ }^{18} \mathrm{FDG}$ injection animals were anaesthetized by $3 \%$ isoflurane with a dedicated small animal anesthesia device and whole body PET scans (10-min static PET scans) were acquired using the preclinical nanoScan PET/MRI system (Mediso Ltd., Hungary). To prevent movement, animals were fixed to a mouse chamber (MultiCell Imaging Chamber, Mediso Ltd., Hungary) and positioned in the center of field of view (FOV). For the determination of the anatomical localization of the organs and tissues, T1-weighted MRI scans were performed (3D GRE EXT multi-FOV; TR/TE 15/2 ms; FOV 70 mm; NEX 2). PET volumes were reconstructed using a three-dimensional Ordered Subsets Expectation Maximization (3D-OSEM) algorithm (TeraTomo, Mediso Ltd., Hungary). PET and MRI images were automatically co-registered by the PET/MRI instrument's acquisition software (Nucline). Reconstructed, reoriented and coregistered images were further analyzed with InterView ${ }^{\mathrm{TM}}$ FUSION (Mediso Ltd., Hungary) dedicated image analysis software. Radiotracer uptake was expressed in terms of standardized uptake values (SUVs). Ellipsoidal 3-dimensional Volumes of Interest (VOI) were manually drawn around the edge of the tissue or organ activity by visual inspection using InterView ${ }^{\mathrm{TM}}$ FUSION multi-modal visualization and evaluation software (Mediso Ltd., Hungary). The standardized uptake value (SUV) was calculated as follows: SUV = [VOI activity $(\mathrm{Bq} / \mathrm{ml})] /[$ injected activity $(\mathrm{Bq}) /$ animal weight $(\mathrm{g})]$, assuming a density of $1 \mathrm{~g} / \mathrm{ml}$. SUVmean is the average SUV value within the volume of interest (VOI).

\subsection{Statistical analysis}

Statistical evaluations were performed by either one-way ANOVA and NewmanKeuls post-test or unpaired t-test (GraphPad Software Inc., USA). All data are presented as means \pm SEM. 


\section{RESULTS}

\subsection{Body composition of the Compact mice}

The body weights of the congenic wild-type mice carrying the wild-type myostatin gene in Compact genetic background were higher than BALB/c mice, but they were smaller than the Compact animals (Table 2). We showed by MRI (magnetic resonance imaging) analysis that profound differences exist in body composition between the genotypes. The qualitative analysis of T1-weighted MRI images revealed remarkable enlargement of skeletal muscle tissues in Compact mice. In the MRI images of congenic wild-type animals, the spaciousness of fat tissues was clearly visualized (Figure 7A). The gross enhancement of hindlimb muscle mass was observable (Figure 7B).

A

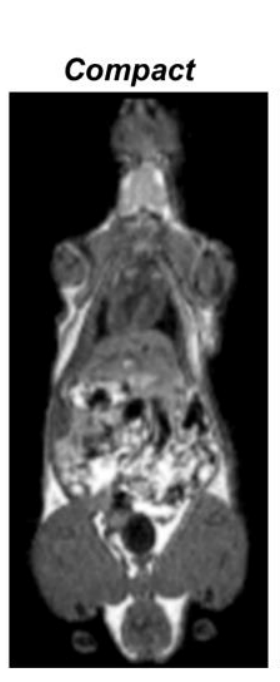

B

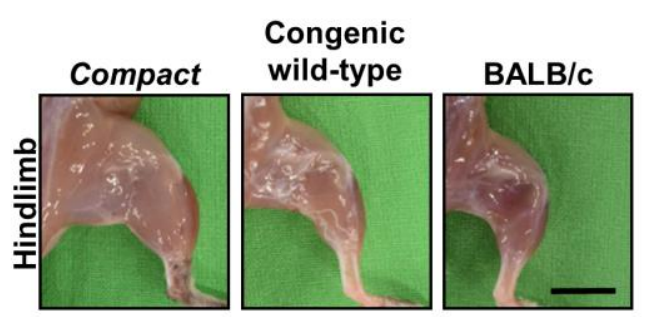

Figure 7. Characterization of muscle phenotype of Compact, congenic wild-type and BALB/c mice.

(A) Representative T1-weighted small animal MRI images of Compact, congenic wild-type and BALB/c mouse. (B) Representative hindlimbs of 4-month old mice showing the different muscularity of mice strains. Bar: 10 $\mathrm{mm}$

The absolute weights of individual hindlimb muscles such as tibialis anterior, quadriceps femoris and gastrocnemius muscles were almost 2-times greater in Compacts compared with congenic wild-type animals, and they were bigger in congenic wild-type animals than in BALB/c mice (Table 2). The muscle weight/body weight ratios showed the highest values in Compacts and the lowest in congenic wild-type mice (Table 2). 
Table 2. Absolute and normalized muscle weights

\begin{tabular}{|c|c|c|c|}
\hline & Compact & Congenic wild-type & BALB/c \\
\hline \multicolumn{4}{|l|}{ 3-4 months old } \\
\hline Body weight, g & $47.4 \pm 0.67^{* * *}, \# \#$ & $40.8 \pm 1.76^{\# \# \#}$ & $25.9 \pm 0.48$ \\
\hline \multicolumn{4}{|l|}{ Muscle weight, mg } \\
\hline TA & 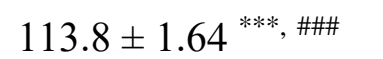 & $67.6 \pm 4.70^{\# \# \#}$ & $45.9 \pm 0.88$ \\
\hline GAST & 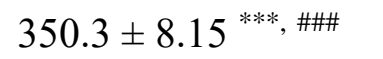 & $183.9 \pm 5.81^{\# \# \#}$ & $140.5 \pm 2.14$ \\
\hline QF & 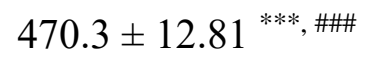 & $246.1 \pm 6.71^{\# \#}$ & $204.6 \pm 3.65$ \\
\hline \multicolumn{4}{|c|}{ Muscle weight/body weight, mg/g } \\
\hline $\mathrm{TA} / \mathrm{bw}$ & $2.4 \pm 0.04$ *** \#\#\# & $1.6 \pm 0.05^{\#}$ & $1.8 \pm 0.04$ \\
\hline GAST/bw & $7.4 \pm 0.13^{* * *}, \# \#$ & $4.5 \pm 0.11^{\# \# \#}$ & $5.4 \pm 0.08$ \\
\hline QF/bw & $9.9 \pm 0.22^{* * *}, \# \#$ & $6.1 \pm 0.22^{\# \# \#}$ & $7.9 \pm 0.11$ \\
\hline \multicolumn{4}{|l|}{10 months old } \\
\hline Body weight, g & $50.4 \pm 0.53^{* *}, \# \#$ & $45.1 \pm 1.29^{\# \# \#}$ & $31.4 \pm 0.88$ \\
\hline \multicolumn{4}{|l|}{ Muscle weight, mg } \\
\hline TA & $141.8 \pm 7.18^{* * * *, \# \#}$ & $69.5 \pm 2.45^{\#}$ & $54.3 \pm 2.45$ \\
\hline GAST & 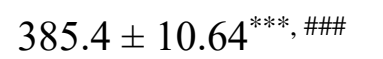 & $185.0 \pm 2.45^{\#}$ & $160.5 \pm 6.52$ \\
\hline QF & $476.0 \pm 14.09^{* * *}, \# \#$ & $247.5 \pm 4.78$ & $221.6 \pm 9.43$ \\
\hline \multicolumn{4}{|c|}{ Muscle weight/body weight, mg/g } \\
\hline $\mathrm{TA} / \mathrm{bw}$ & $2.8 \pm 0.14^{* * *}$ & $1.56 \pm 0.08$ & $1.7 \pm 0.07$ \\
\hline GAST/bw & 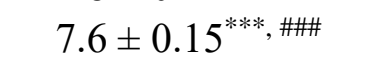 & $4.13 \pm 0.09^{\# \# \#}$ & $5.1 \pm 0.11$ \\
\hline $\mathrm{QF} / \mathrm{bw}$ & 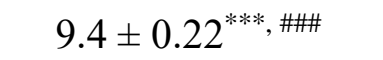 & $5.52 \pm 0.12^{\# \# \#}$ & $7.0 \pm 0.12$ \\
\hline \multicolumn{4}{|c|}{$\begin{array}{l}\text { Values are means } \pm \text { SEM (3- to 4-mo-old animals: } n=\overline{9 \text { Compact, } 7 \text { congenic wild }} \text {-type, and } 8 \mathrm{BALB} / \mathrm{c} \text { mice; } \\
\text { 10-mo-old animals: } n=8 \text { Compact, } 12 \text { congenic wild-type, and } 8 \mathrm{BALB} / \mathrm{c} \text { mice). The values of the Compact } \\
\text { group are significantly different from the congenic wild-type group group at } P^{*}<0.05 ; P^{* *}<0.01 ; P^{* * *}<0.001 \text {. }\end{array}$} \\
\hline \multicolumn{4}{|c|}{ 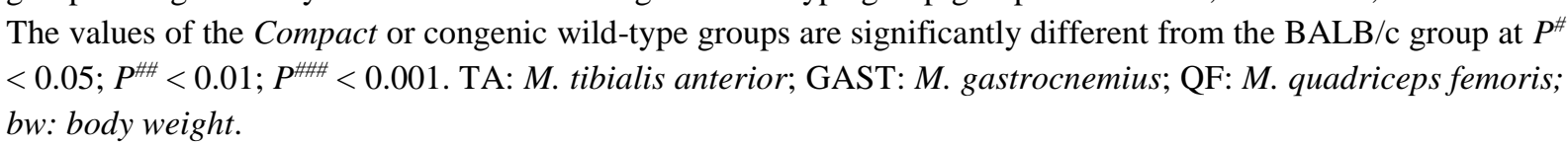 } \\
\hline
\end{tabular}

We found that the absolute weight of heart and kidney of the Compact and congenic wildtype mice were comparable and higher than that of BALB/c. The absolute weight of abdominal fat increased by $\sim 30 \%$ in 3-4 months old and $~ 50 \%$ in 10 months old congenic wild-type compared with Compacts mice, and it was markedly lower in BALB/c group than in Compacts (Table 3). Liver/body weight ratio of Compacts was smaller than that of congenic wild-type animals in both ages (Table 3). Abdominal fat/body weight ratios were comparable in Compact and BALB/c animals, and it was almost 2-fold higher in congenic wild-type mice (Table 3). The weights of heart and kidney in proportion to body weight were the smallest in Compacts and highest in BALB/c mice (Table 3). 
Table 3. Absolute and normalized organ weights

\begin{tabular}{|c|c|c|c|}
\hline & Compact & Congenic wild-type & BALB/c \\
\hline \multicolumn{4}{|c|}{ 3-4 months old } \\
\hline \multicolumn{4}{|c|}{ Organ weight, mg } \\
\hline Liver & $1792.0 \pm 43.45$ & $1874.0 \pm 33.68^{\# \# \#}$ & $1158.0 \pm 31.55$ \\
\hline Abd. fat & $672.8 \pm 59.07^{* *}, \# \#$ & $1042.0 \pm 105.30^{\# \# \#}$ & $271.9 \pm 20.21$ \\
\hline Heart & $153.8 \pm 4.71 \#$ & $163.1 \pm 11.41^{\# \#}$ & $121.4 \pm 3.17$ \\
\hline Kidney & $237.8 \pm 5.97^{\# \# \#}$ & $250.6 \pm 11.76^{\# \# \#}$ & $190.4 \pm 5.42$ \\
\hline \multicolumn{4}{|c|}{ Organ weight/body weight, mg/g } \\
\hline Liver/bw & $37.8 \pm 0.81 * * *$ & $46.3 \pm 1.41$ & $44.72 \pm 1.04$ \\
\hline Abd. fat/bw & $14.5 \pm 1.30^{* * *}$ & $25.3 \pm 1.76^{\# \# \#}$ & $10.55 \pm 0.89$ \\
\hline Heart/bw & $3.3 \pm 0.13^{* * *}, \# \#$ & $3.9 \pm 0.12^{\# \# \#}$ & $4.68 \pm 0.08$ \\
\hline Kidney/bw & $5.0 \pm 0.11^{* * *}, \# \#$ & $6.16 \pm 0.17^{\# \# \#}$ & $7.35 \pm 0.17$ \\
\hline \multicolumn{4}{|c|}{10 months old } \\
\hline \multicolumn{4}{|c|}{ Organ weight, mg } \\
\hline Liver & $2037.0 \pm 43.15^{*}, \# \#$ & $2213.0 \pm 65.05^{\# \# \#}$ & $1525.0 \pm 49.64$ \\
\hline Abd. fat & $683.5 \pm 35.04^{* *}$ & $1236.0 \pm 156.3^{\# \# \#}$ & $374.6 \pm 41.99$ \\
\hline Heart & $173.0 \pm 3.21^{\# \# \#}$ & $178.2 \pm 5.67^{\# \# \#}$ & $140.3 \pm 5.11$ \\
\hline Kidney & $266.9 \pm 3.63$ & $300.3 \pm 12.03$ & $290.6 \pm 21.00$ \\
\hline \multicolumn{4}{|c|}{ Organ weight/body weight, mg/g } \\
\hline Liver/bw & $40.4 \pm 0.80^{* * *}$ & $49.3 \pm 1.29$ & $48.6 \pm 0.82$ \\
\hline Abd. fat/bw & $13.6 \pm 0.75^{* * *}$ & $26.8 \pm 2.68^{\# \# \#}$ & $12.1 \pm 1.48$ \\
\hline Heart/bw & $3.4 \pm 0.07^{* * *}$ & $3.9 \pm 0.07^{\# \# \#}$ & $4.5 \pm 0.11$ \\
\hline Kidney/bw & $5.3 \pm 0.10^{*}, \ldots \#$ & $6.6 \pm 0.11^{\# \# \#}$ & $9.3 \pm 0.73$ \\
\hline
\end{tabular}

Values are means \pm SEM (3- to 4-mo-old animals: $n=9$ Compact, 7 congenic wild-type, and 8 BALB/c mice; 10-mo-old animals: $n=8$ Compact, 12 congenic wild-type, and 8 BALB/c mice). The values of the Compact group are significantly different from the congenic wild-type group at $P^{*}<0.05 ; P^{* *}<0.01 ; P^{* * *}<0.001$. The values of Compact or congenic wild-type groups are significantly different from the BALB/c group at $P^{\#}<0.05$;

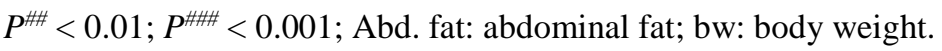

\subsection{Tissue glycogen and protein distribution}

Myostatin was reported to influence the glycogen content of C2C12 myoblasts [46], and muscle glycogen was reduced in type 2 diabetes mellitus [79]. The Compact line was selected for high protein content and hypermuscularity [65]. Beside the increased protein amount the higher glycogen content may account for the increased muscle weight of the Compact mice. To distinguish the role of Compact mutation and the Compact genetic background in the regulation of glycogen stores here we compared the glycogen content of Compact, congenic wild-type and BALB/c muscles. We found that total glycogen levels of Compact muscles were the highest, the congenic wild-type and BALB/c samples contained 
comparable and smaller amount of glycogen (Figure 8A). However, the glycogen concentration was the highest BALB/c mice and displayed no differences between Compact and congenic wild-type groups (Figure 8B).

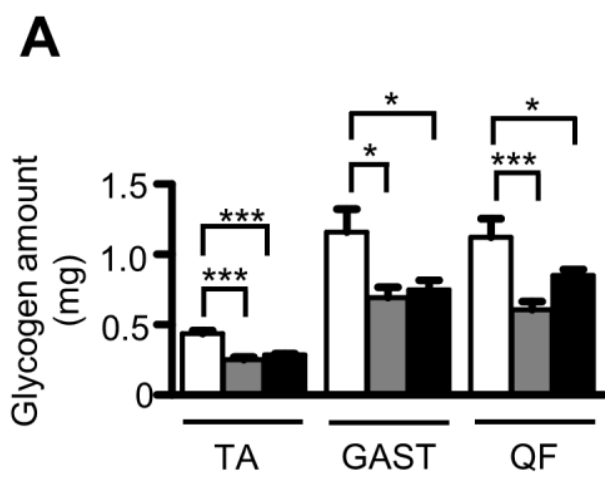

B

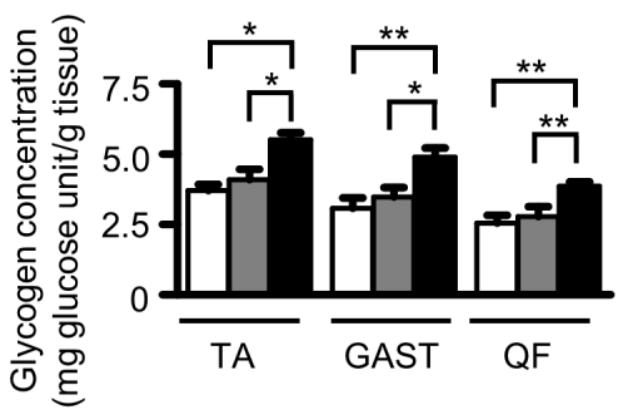

$\square$ Compact $\square$ Congenic wild-type $\square$ BALB/c

Figure 8. Characterization of the Compact, congenic wild-type and BALB/c livers. Total glycogen amount $(A)$ and glycogen concentration (glycogen amount/muscle weight) of hindlimb muscles $(B)$. Data are presented as means $\pm \mathrm{SEM} ; n=3$ Compact, 6 congenic wild-type, and 4 BALB/c mice [m. tibialis anterior (TA)]; $n=6$ Compact, 5 congenic wild-type, and $8 \mathrm{BALB} / \mathrm{c}$ mice [m. gastrocnemius (GAST)]; $n=4$ Compact, 6 congenic wild-type and $9 \mathrm{BALB} / \mathrm{c}$ mice $[m$. quadriceps femoris $(\mathrm{QF})] . P^{*}<0.05 ; P^{* *}<0.01 ; P^{* * *}<0.001$.

Both the myostatin mutation and Compact genetic background influenced liver weight, and liver functions as a glycogen store; therefore, we measured the glycogen content of the liver samples. Analyzing the total glycogen amount and glycogen concentration we did not observe differences between congenic wild-type and Compact groups; the glycogen content/liver weight ratio of BALB/c animals was more than 2.5-fold smaller (Figure 9A). The visualization of glycogen by PAS-staining verified the results of spectrophotometry, weaker staining was observed in BALB/c samples (Figure 9B). The Compact mice were selected for high protein content, and Sawitzky and colleagues demonstrated that the selection for high protein mass also selects for high muscle glycogen [80]; thus, we determined the total protein amount in the investigated muscles. We found that the total protein content of the Compact muscles were the highest and the congenic wild-type and BALB/c samples contained smaller amount of protein in accordance to total glycogen levels (Table 4). Furthermore, we measured the total hepatic protein content and we found that it was the highest in Compacts and lowest in the congenic wild-type group (Table 4). 

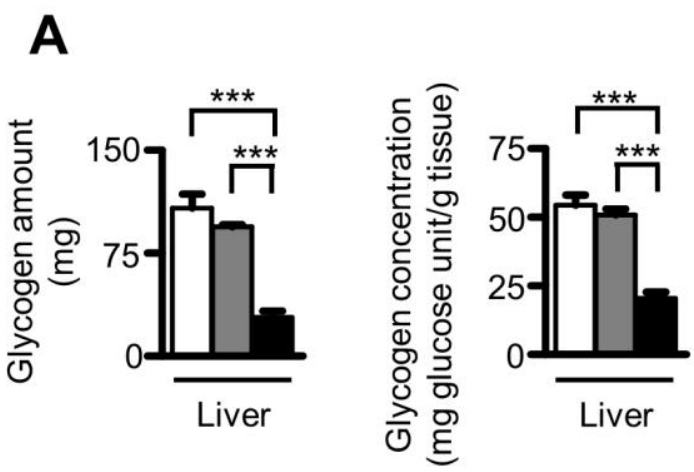

B

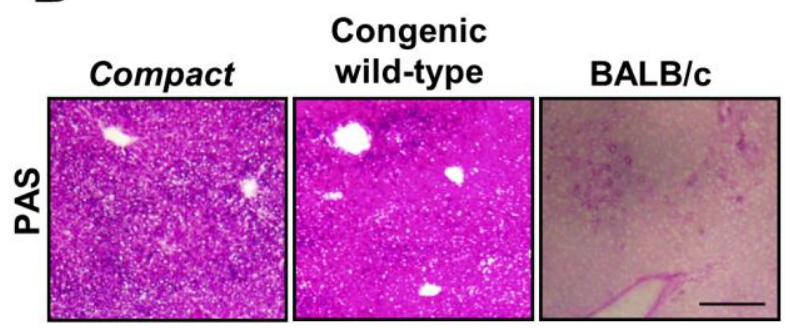

$\square$ Compact $\square$ Congenic wild-type

Figure 9. Characterization of the Compact, congenic wild-type, and BALB/c livers. A: total glycogen amount and glycogen concentration of the liver samples determined by spectrophotometry $(n=4$ Compact, 5 congenic wild-type, and $4 \mathrm{BALB} / \mathrm{c}$ mice). $B$ : representative Periodic Acid-Schiff (PAS)-stained images show glycogen content of the liver. Scale bar, $200 \mu \mathrm{m}$. Data are reported as means \pm SEM. $P^{* * *}<0.001$.

Table 4. Total protein content in hindlimb muscles and liver

\begin{tabular}{lccr}
\hline \hline \multicolumn{1}{c}{ Compact } & Congenic wild-type & BALB/c \\
\hline Total protein content, $\left(\mathrm{g}^{*} 10^{-3}\right)$ & & \\
TA & $19 \pm 0.5^{* * *, \# \#}$ & $9 \pm 0.4$ & $9 \pm 0.4$ \\
GAST & $56 \pm 4.4^{* * *, \# \#}$ & $23 \pm 1.0$ & $19 \pm 0.4$ \\
QF & $64 \pm 2.9^{* * *}, \# \#$ & $35 \pm 2.3$ & $33 \pm 0.7$ \\
Liver & $352 \pm 14.1^{* * *}, \# \#$ & $230 \pm 4.1^{\# \#}$ & $274 \pm 9.4$ \\
\hline
\end{tabular}

Values are means \pm SEM; $n=3$ Compact, 3 congenic wild-type, and 3 BALB/c mice [m. tibialis anterior (TA)]; $n=3$ Compact, 5 congenic wild-type and $3 \mathrm{BALB} / \mathrm{c}$ mice [m. gastrocnemius (GAST)]; $n=3$ Compact, 6 congenic wild-type and $3 \mathrm{BALB} / \mathrm{c}$ mice $[$ m. quadriceps femoris $(\mathrm{QF})] ; n=5$ Compact, 6 congenic wild-type and $8 \mathrm{BALB} / \mathrm{c}$ mice (liver). The values of the Compact group are significantly different from the congenic wild-type group at $P^{* * *}<0.001$. The values of the Compact or congenic wild-type groups are significantly different from

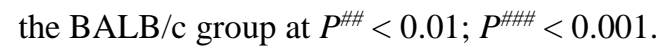

\subsection{Skeletal muscle cellularity in Compact mice}

\subsubsection{Muscle characteristics of Compact mice}

We tested, whether the higher glycogen amount of the Compact muscle is caused by the changes in the glycogen content of the individual muscle fibers. To analyze this, first we determined the average intensity (optical density, OD) of PAS-staining and fiber sizes on the whole CSA of the TA muscle (Figure 10A). The average intensity of PAS-staining did not show significant change in Compact muscle fibers compared to those of the wild type muscle (Figure 10A), and no significant difference was observed regarding the average CSAs of the fibers either (Figure 10B). 
A

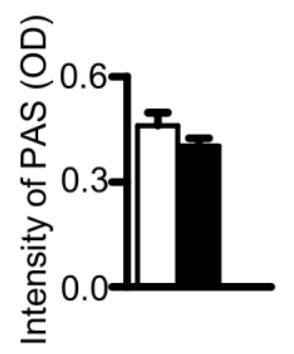

Compact
B

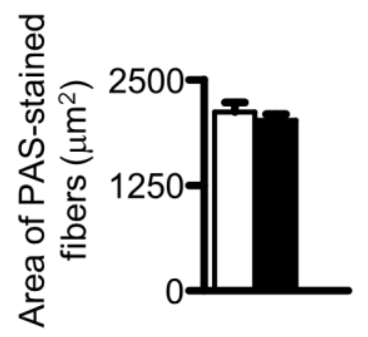

C

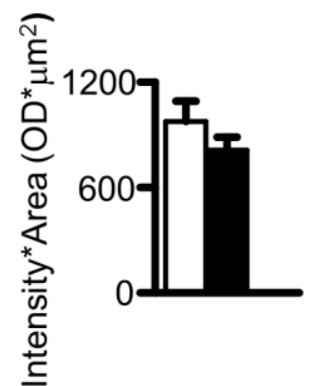

D

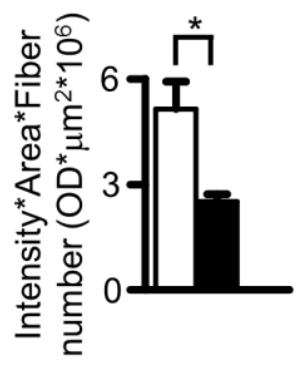

Figure 10. Average intensity of PAS-staining of the fibers on whole muscle cross sectional area $(A)$, average glycogen content of the fibers $(B)$, average area of the fibers $(C)$, and total glycogen content of the TA muscle (D) based on PAS-staining ( $n=3$ Compact and 3 BALB/c mice). Data are reported as means \pm SEM; $P^{*}<0.05$.

The average intensity of PAS-staining multiplied by the value of fiber size is suitable to estimate the average glycogen content of the individual fibers. Comparing the average glycogen content of the fibers we could not detect any significant changes between the two groups of animals (Figure 10C). Similar to previously reported results [71, 72] the fiber number increased 1.7-fold in Compact animals (Table 5). Multiplying the average glycogen content of the individual fibers by the increased fiber number we detected a similar, two-fold increase in the glycogen content of the whole muscle by PAS-staining (Figure 10D) as measured by spectrophotometry. Taken together, the average glycogen content of the individual fibers did not change; therefore, the increased fiber number can explain the elevated glycogen amount of the Compact TA muscle.

\subsubsection{Glycogen content of the different fiber types}

To analyze the possible presence of the fiber-type specific alterations of the glycogen content, first we examined the fiber type composition of TA muscle and measured the CSAs of the different fiber types on serial sections immunostained by antibodies against the different isoforms of MyHCs (Figure 6). Regarding the fiber composition of the Compact muscle, we could not detect the slow MyHCI isoform in TA muscles (Table 5) in accordance with the literature [81]. The ratio of IIB fibers (Table 5) increased 1.6-fold with concomitant decreases of IIX (Table 5) and IIA fibers (Table 5) indicating a glycolytic shift. Because the 
number of IIA fibers in Compact TA was very low (Table 5), we could not perform statistical analysis on this type of fibers.

Table 5. Relative fiber type distribution

\begin{tabular}{ccc}
\hline \hline Item & Compact & BALB/c \\
\hline Total fiber number & $5200 \pm 227.6^{\# \# \#}$ & $3100 \pm 52.48$ \\
Fiber type frequency (\%) & & \\
MyHCI & 0 & 0 \\
MyHCIIA & $1 \pm 0.05^{\# \# \#}$ & $3 \pm 0.09$ \\
MyHCIIX & $19 \pm 3.25^{\#}$ & $47 \pm 2.59$ \\
MyHCIIB & $80 \pm 3.29^{\# \#}$ & $50 \pm 2.55$ \\
\hline
\end{tabular}

Values are mean \pm SEM; $n=3$ Compact and $3 \mathrm{BALB} / \mathrm{c}$ mice. The values of Compact group is significantly different from the BALB/c group at $P^{\#}<0.05 ; P^{\# \#}<0.01 ; P^{\# \# \#}<0.001 ; M y H C$ : Myosin Heavy Chain.

Remarkable differences were observed comparing the sizes of the different fiber types on the whole CSA of the muscle. As in wild type animals, the CSA of type IIB fibers was 1.44-fold larger than those of type IIX; and IIX fibers were 1.52-fold larger than the IIA ones (Figure 11A). In Compact mice we found a similar result: the CSA of type IIB fibers was 1.62-fold bigger than those of IIX fibers (Figure 11A). Comparing the two mice lines we did not find any differences between the average size of either IIB or IIX fibers. The frequency distributions of both the IIX and IIB fiber sizes showed similar shapes in Compact and wild type mice (Figure 11B, C). The histograms of IIX fibers (Figure 11B) revealed right-skewed distribution in both groups of animals. In case of IIB fibers the bellshaped curves were wider in Compact and BALB/c animals (Figure 11C) than those of IIX fibers (Figure 11B).

The superficial and deep regions of the TA muscle consist of different fiber types [78, 82]; therefore, we performed our measurements on both regions as well. According to fiber size we found similar results, i.e., the area of IIB fibers was the greatest (Figure 12A), with no differences between the superficial and deep regions of the TA muscle in either mouse line, and no changes were observed regarding the fiber sizes of the two mice strains (Figure 12A).

To compare the effect of the Compact mutation on the fiber-type specific glycogen content of the muscle, we measured the average intensity of PAS-staining of the different fiber types on serial cryosections stained with antibodies against the different types of MyHC isoforms and PAS, respectively (Figure 6). 

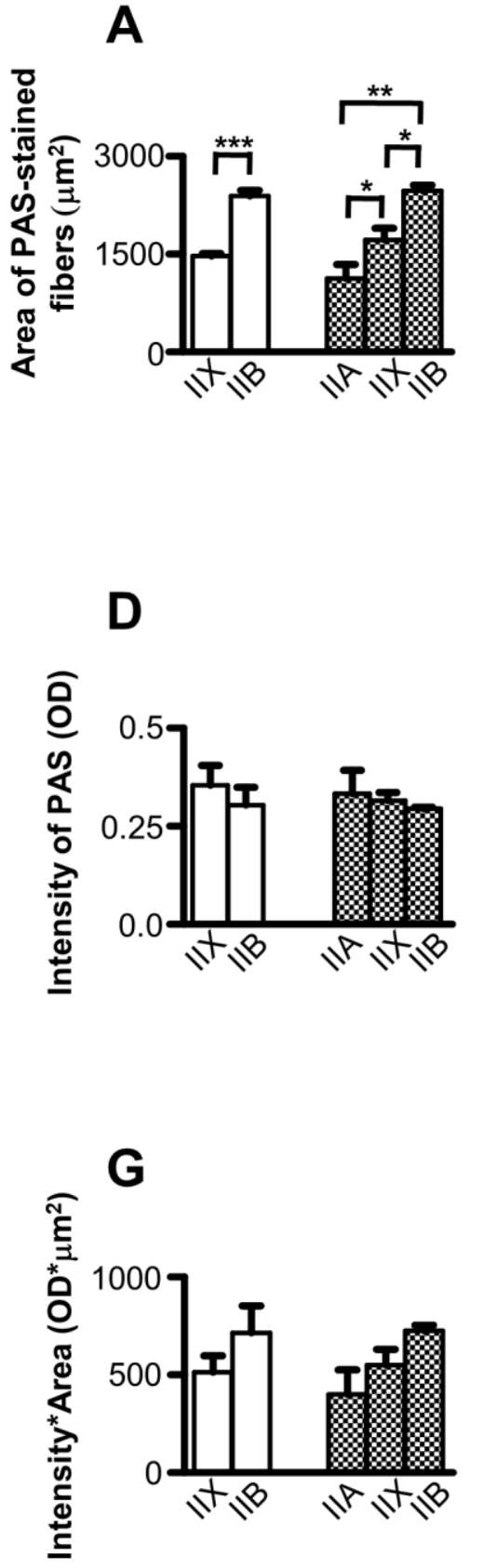

B

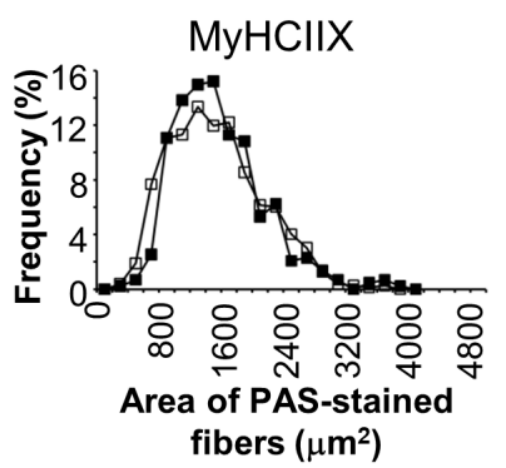

E

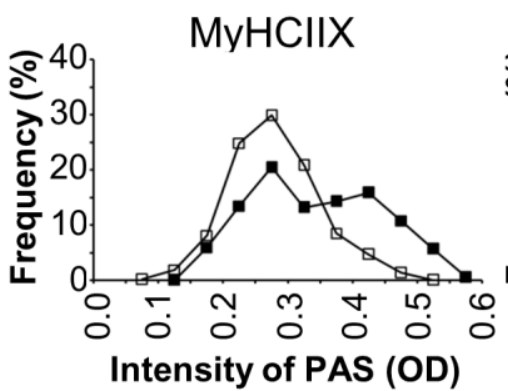

\section{H}

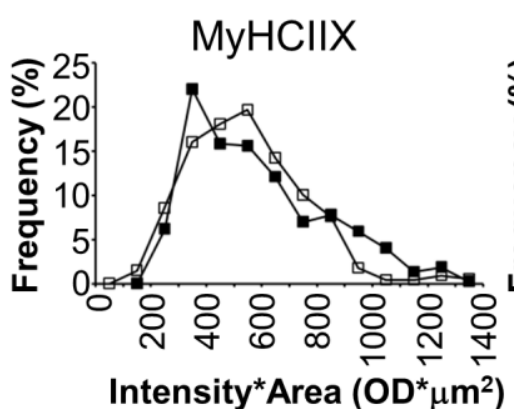

\section{C}

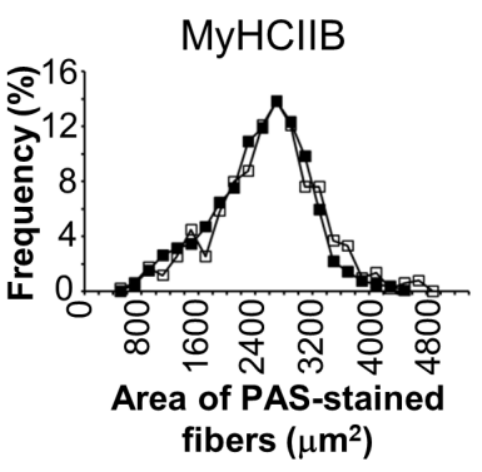

F

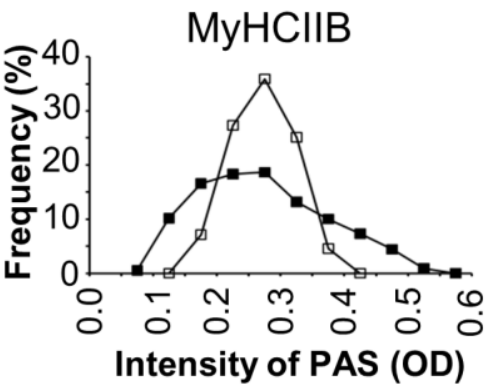

I

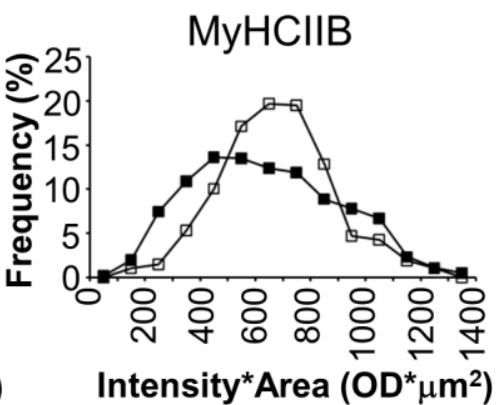

\section{Compact}

BALB/c

$\rightarrow$ Compact

巴 BALB/c

Figure 11. Average area of fibers $(A)$, average intensity of PAS-staining $(D)$ and average glycogen content of the different fiber types $(G)$ on the whole cross sectional area of the tibialis anterior muscle in the Compact and $\mathrm{BALB} / \mathrm{c}$ mice $(n=3$ Compact and $3 \mathrm{BALB} / \mathrm{c}$ mice). Histograms $(B, C, E, F, H, I)$ show the frequency distribution of the defined parameters. Data are reported as means \pm SEM; $P^{*}<0.05 ; P^{* *}<0.01 ; P^{* * *}<0.001$.

The average intensity of PAS-staining showed a similar decreasing tendency in a rank order IIA>IIX>IIB on the whole CSA of the muscle: IIB fibers exhibited the weakest OD 
(Figure 12B), as in the superficial and deep regions (Figure 12B) in both mouse lines. The average intensity of PAS-staining of the different fiber types did not show significant differences between the Compact and BALB/c fibers on the whole muscle CSA (Figure 11D), and no significant changes could be observed comparing the PAS intensity of the fiber types in the superficial and deep regions either (Figure 12B). However, the frequency distribution revealed differences despite the similar average values of intensity of PASstaining in Compact and wild type mice: the Compact TA muscle contained populations of IIX fibers with higher, and IIB fibers with both higher and lower intensity of PAS-staining resulting in a wider distribution in mutant animals (Figure 11E, F).

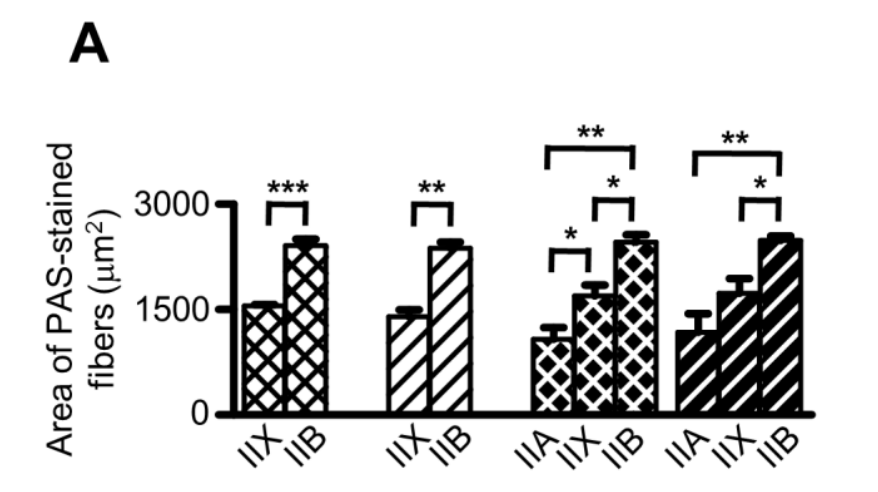

B
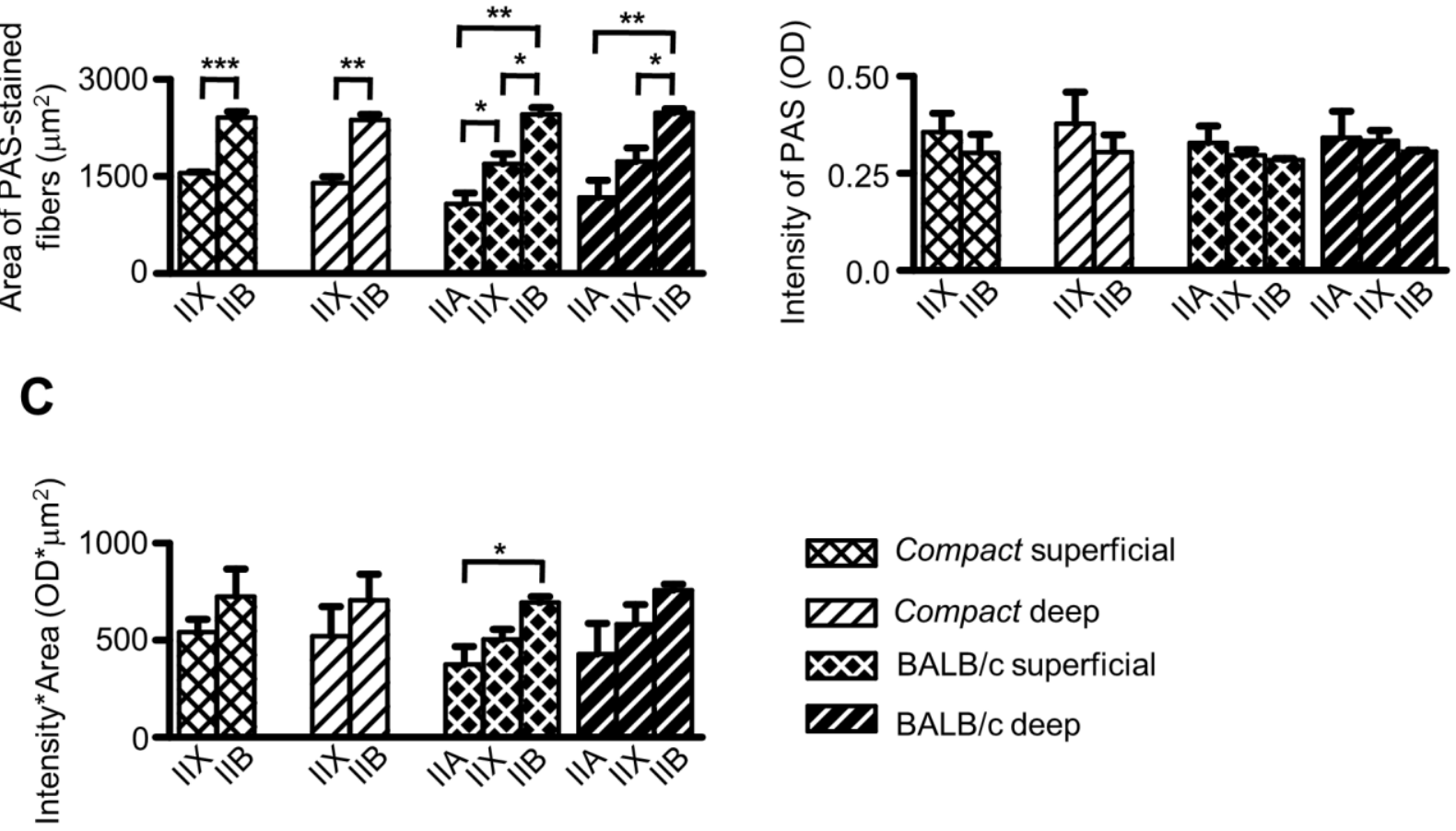

Figure 12. Average area of fibers $(A)$, intensity of PAS-staining $(B)$ and average glycogen content of the fiber types $(C)$ in the superficial and deep regions of the tibialis anterior muscle in Compact and BALB/c mice $(n=3$ Compact and $3 \mathrm{BALB} / \mathrm{c}$ mice). Data are reported as mean $\pm \mathrm{SEM} ; P^{*}<0.05 ; P^{* *}<0.01 ; P^{* * *}<0.001$.

Next, the average glycogen content of the different fiber types was compared in both strains. The average glycogen content of the IIB fibers was greater than those of IIX fibers, whereas the IIA was the lowest in the superficial and deep regions of wild type muscle (Figure 12C). The results indicated significant difference in the superficial region of BALB/c animals (Figure 12C). The fiber type specific glycogen content on the whole CSA revealed 
similar tendency; however, without any significant changes (Figure 11G). In parallel with the frequency distribution of PAS intensity (Figure 11E, F), histograms of the average glycogen content (Figure 11H, I) showed that the Compact TA muscle contains a population of IIX fibers with higher, and IIB fibers with both higher and lower glycogen content. Finally, we compared the total glycogen content of the different fiber types in the TA muscle. The glycogen index of IIB fibers was the highest, so that the IIB fibers stored the most glycogen in both BALB/c and Compact mice. The glycogen index value of IIX fibers was 2.7-fold larger in BALB/c mice compared to Compact animals (Figure 13) in line with the higher number of IIX fibers present in BALB/c mice. In contrast the glycogen index of IIB fibers was 1.5-fold bigger in Compacts in good accordance with the increased proportion of IIB fibers in Compact mice.

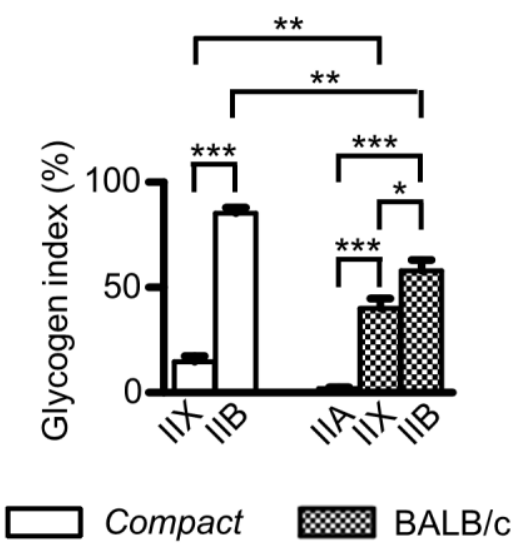

Figure 13. Glycogen index of the different (IIA, IIX and IIB) fiber types of the tibialis anterior muscle in $\mathrm{BALB} / \mathrm{c}$ and Compact mice $\left(\mathrm{n}=3\right.$ Compact and $3 \mathrm{BALB} / \mathrm{c}$ mice). Data are reported as means $\pm \mathrm{SEM} ; P^{*}<$ $0.05 ; P^{* *}<0.01 ; P^{* * *}<0.001$.

\subsection{Metabolic alterations in Compact mice}

\subsubsection{Glucose tolerance and insulin sensitivity are improved by Compact myostatin} mutation and reduced by Compact genetic background

As reported previously, knocking out of myostatin increases glucose tolerance and insulin sensitivity [40, 41], and fed and fasting glucose levels in myostatin knock out mice were not significantly different from controls [36]. Since the Compact mice were hypermuscular and had reduced abdominal fat, we examined whether Compact mice showed alterations in glucose metabolism. Because age is reported to influence glucose tolerance [83, 84], we compared whole body glucose tolerance and insulin sensitivity from both young (3-4 months old) and middle-aged (10 months old) groups of mice. 
Table 6. Fasting blood glucose levels

\begin{tabular}{|c|c|c|c|}
\hline & Compact & Congenic wild-type & BALB/c \\
\hline \multicolumn{4}{|c|}{ Fasting blood glucose (mM/l) } \\
\hline 3-4 months old & $5.81 \pm 0.67$ & $5.81 \pm 0.64$ & $5.38 \pm 0.69$ \\
\hline 10 months old & $5.20 \pm 0.25$ & $5.07 \pm 0.16$ & $4.82 \pm 0.28$ \\
\hline
\end{tabular}

Our results did not show any significant changes in fasting blood glucose levels comparing Compact mice with age-matched congenic wild-type and BALB/c animals (Table 6); however, the response to exogenous glucose revealed differences between genotypes. Compact and $\mathrm{BALB} / \mathrm{c}$ mice showed greater glucose tolerance compared with age-matched congenic wild-type strain. The area under the curve of blood glucose concentrations during the glucose tolerance test was significantly higher in congenic wild-type mice compared to age-matched Compact and BALB/c (Figure 14A, B). We performed insulin tolerance tests to measure blood glucose changes following insulin administration. Insulin treatment reduced blood glucose levels in all groups of mice, indicating the insulin responsivity. Congenic wildtype mice showed weaker insulin sensitivity compared with Compact and BALB/c groups. The area under the curve value during insulin tolerance test was significantly higher in congenic wild-types than in Compact and BALB/c mice at both ages (Figure 14C, D). Both glucose tolerance and insulin sensitivity tests showed comparable results between agematched Compacts and BALB/c mice (Figure 14A-D).

To test the effect of aging on glucose tolerance and insulin sensitivity, the area under the curve values were compared. The area under the curve of blood glucose concentrations during glucose tolerance tests of 10 months old Compacts was significantly higher than that of 3-4 months old animals $(1154 \pm 31.5$ vs $943.3 \pm 86.3 ; P=0.0318)$; no alterations were observed comparing young and middle-aged congenic wild-type or BALB/c groups. The area under the curve values during insulin tolerance test of young animals were not significantly different from middle-aged groups, although they tended to be smaller in all three genotypes. 
A

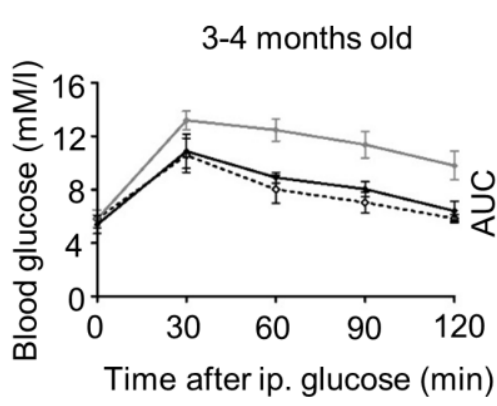

C

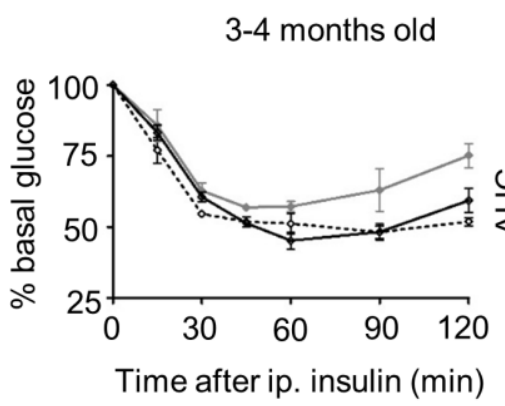

B
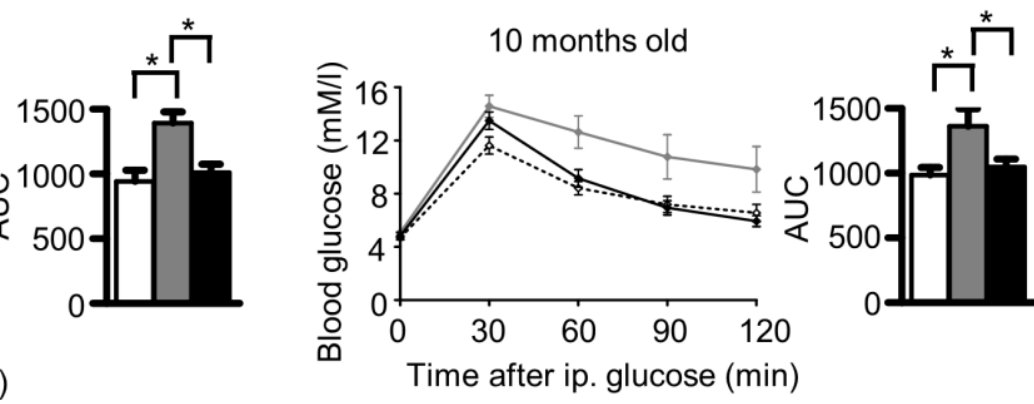

D

10 months old
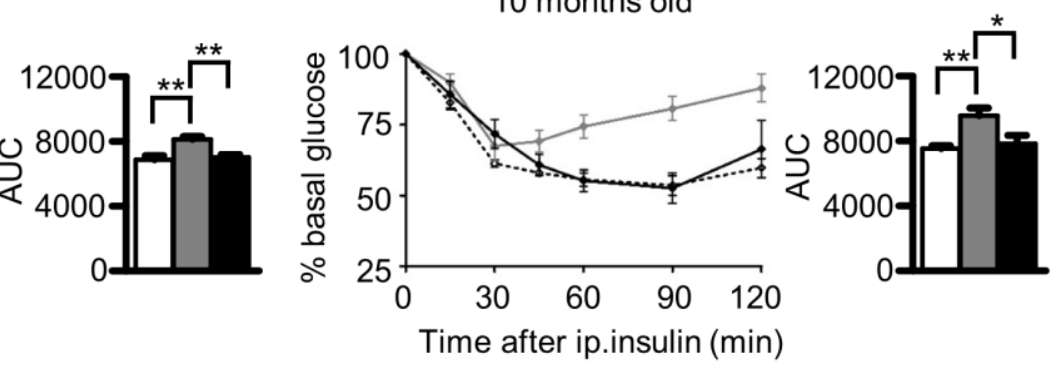

\section{$\bullet$ - Compact $\leadsto$ Congenic wild-type $\leadsto$ BALB/c \\ $\square$ Compact $\square$ Congenic wild-type $\square$ BALB/c}

Figure 14. Glucose tolerance and insulin sensitivity are improved by Compact myostatin mutation and reduced in congenic wild-type mice. Intraperitoneal (ip) glucose tolerance $(A$ and $B)$ and insulin sensitivity tests $(C$ and $D$ ) of 3- to 4-mo-old $(A$ and $C$ ) and 10-mo-old animals $(B$ and $D)$. Area under the curve (AUC) values are presented in bar diagrams. $n=3$ Compact, 7 congenic wild-type, and $3 \mathrm{BALB} / \mathrm{c}$ mice $(A), n=7$ Compact, 3 congenic wild-type, and $6 \mathrm{BALB} / \mathrm{c}$ mice $(B), n=3$ Compact, 3 congenic wild-type, and $4 \mathrm{BALB} / \mathrm{c}$ mice $(C)$, and $n=6$ Compact, 4 congenic wild-type and 4 BALB $/ \mathrm{c}$ mice $(D)$. Data are reported as means \pm SEM; $P^{*}<0.05 ; P^{* *}$ $<0.01$.

\subsubsection{The Compact mutation increases ${ }^{18} \mathrm{FDG}$ uptake in skeletal muscle, liver, and} adipose tissue

The Compact mutation and genetic background affected glucose tolerance and insulin sensitivity; therefore, we evaluated glucose uptake in different tissues with known insulin responsiveness using small-animal PET/MRI imaging. By the quantitative analysis of decaycorrected ${ }^{18}$ FDG-PET images we found significant differences in the SUVmean of the selected organs $50 \mathrm{~min}$ after tracer injection (Figure 15). The ${ }^{18} \mathrm{FDG}$ accumulation of the skeletal muscle was comparable in Compacts and BALB/c animals, and moderate uptake was observed in congenic wild-type animals. The radiotracer uptake of white adipose tissue showed similar results as skeletal muscle, mild uptake was observed in the congenic wild-type 
mice, and it was approximately two-fold higher in Compact and BALB/c animals. The liver of Compact mice showed the highest SUVmean, which was followed by the congenic wildtype and $\mathrm{BALB} / \mathrm{c}$ groups.

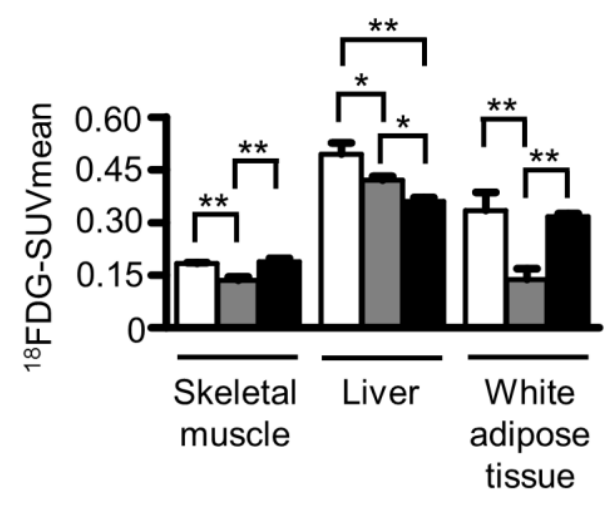

$\square$ Compact $\square$ Congenic wild-type $\square$ BALB/c

Figure 15. Compact mutation increases ${ }^{18} \mathrm{FDG}$ (2-deoxy-2-[ $\left[{ }^{18} \mathrm{~F}\right]$ fluoro-D-glucose) uptake. Quantitative analysis of ${ }^{18} \mathrm{FDG}$ uptake of selected tissues in Compact, congenic, and BALB/c mice $50 \mathrm{~min}$ after tracer injection. Data are presented as means $\pm \mathrm{SEM} ; n=3$ Compact, 4 congenic wild-type, and $4 \mathrm{BALB} / \mathrm{c}$ mice (skeletal muscle, liver); $n=4$ in each group (white adipose tissue). $P^{*}<0.05 ; P^{* *}<0.01$.

\subsubsection{Liver characteristics in Compact mice}

It was reported that knocking out of myostatin results in comparable absolute liver weight, lower liver/body weight ratio and decreased ALT (alanine aminotransferase) activity of the liver [37]. We found that liver mass and liver/body weight ratios are not increased in proportion to skeletal muscle mass in Compact mice; therefore, we aimed to assess whether ALT activity in proportion to body weight is also reduced similarly to myostatin knock out animals.

Our data show that total liver ALT activity/body weight ratio was the lowest in Compacts and was the biggest in BALB/c mice (Figure 16).

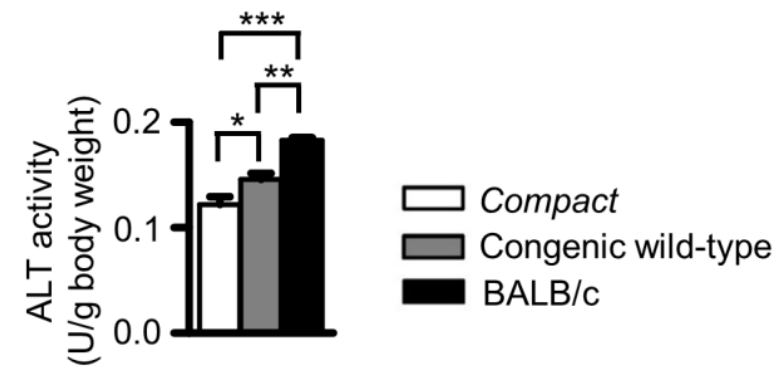

Figure 16. Hepatic ALT activity. Values are means \pm SEM $\left(\mathrm{n}=4\right.$ mice in each group). $P^{*}<0.05 ; P^{* *}<0.01$; $P^{* * *}<0.001$. 
Next we administered glucose precursor pyruvate to measure hepatic gluconeogenesis. The intraperitoneal pyruvate tolerance test showed that hepatic gluconeogenesis increased in Compact and congenic wild-type animals compared to BALB/c animals. The area under the curve of blood glucose concentrations during pyruvate tolerance test was comparable in Compact and congenic wild-type mice, and it was lower in BALB/c animals (Figure 17). The area under the curve of blood glucose concentrations during pyruvate tolerance test was comparable in Compact and congenic wild-type mice, and it was lower in BALB/c animals (Figure 17).
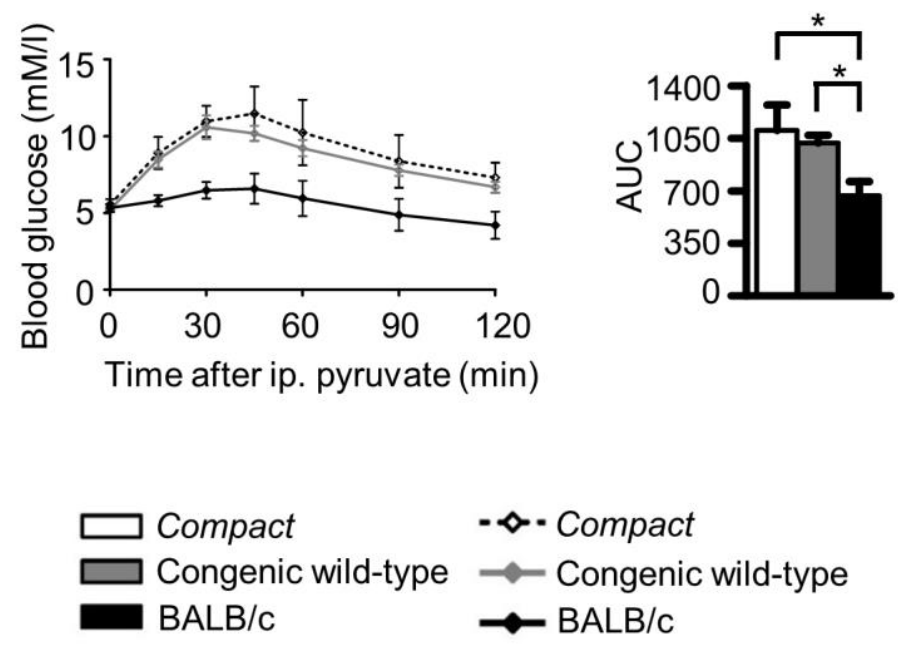

Figure 17. Intraperitoneal (ip) pyruvate tolerance test of 3- to 4-mo-old mice ( $n=3$ Compact, 5 congenic wildtype and $4 \mathrm{BALB} / \mathrm{c}$ mice). Area under the curve (AUC) values are presented in bar diagrams. Data are reported as means \pm SEM; $P^{*}<0.05$.

\subsection{Molecular consequences of the Compact mutation}

\subsubsection{The Compact mutation of myostatin propeptide decreases myostatin formation}

The Compact Mstn(Cmpt-dllAbc) mutation in the myostatin gene eliminates amino acids 224-228 in the propeptide and creates a new Phe residue [3]. The mutation is toward the $\mathrm{N}$-terminus from the furin cleavage site, since promyostatin is proteolyticaly processed by furin at the RSRR (263-266) site to give the active processed myostatin [7]. The Compact mutation is a non-frameshift 12-bp deletion and the biologically active growth factor domain is intact; therefore, functional myostatin formation should be permitted. The presence of Compact mutation was verified by sequencing the myostatin gene (data not shown). Importantly, we were able to detect mature myostatin dimer in Compact skeletal muscle (Figure 18). 


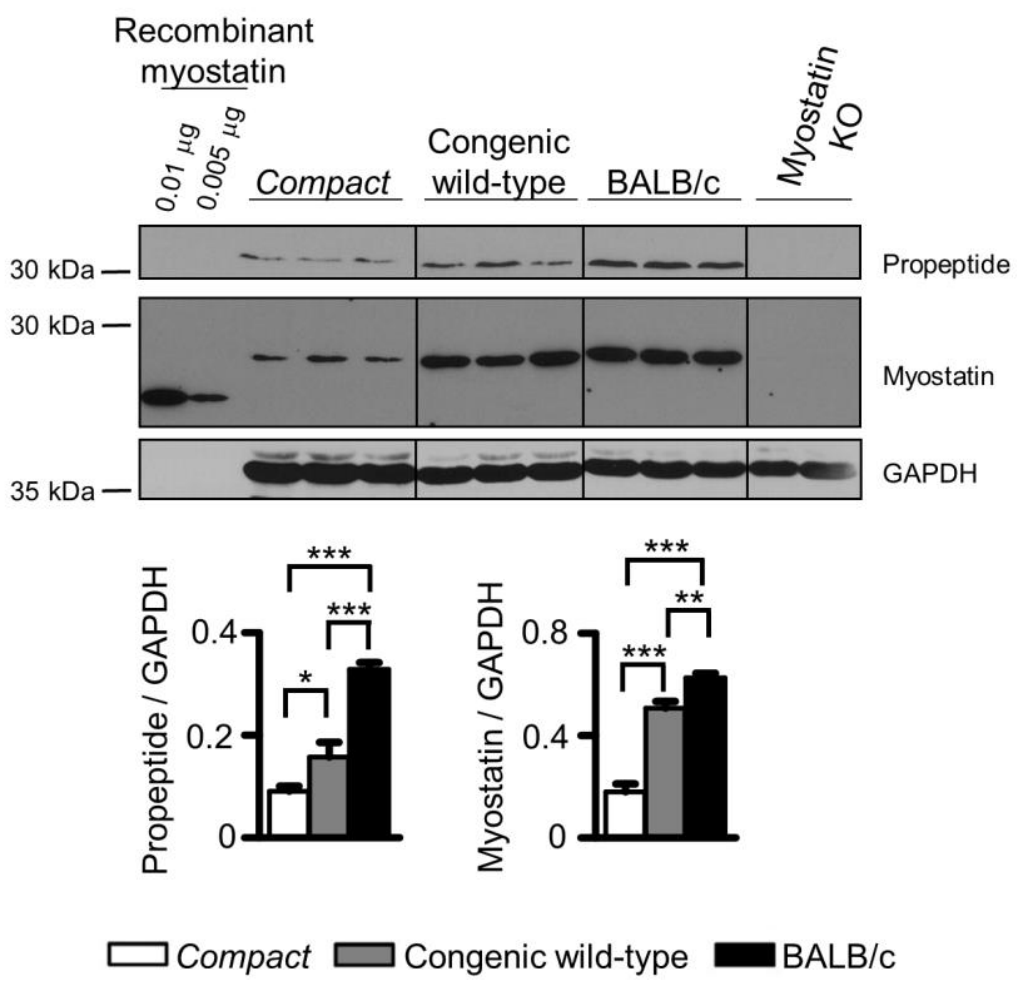

Figure 18. Myostatin level in skeletal muscle of Compact, congenic wild-type and BALB/c mice. $M$. gastrocnemius protein extracts were subjected to SDS-PAGE and blotted with anti-myostatin or anti-propeptide antibody. Representative images are shown. Note the presence of mature myostatin dimer and myostatin propeptide in Compact samples. Mouse recombinant myostatin was used as a positive control, and muscle homogenates of myostatin knock out (KO) mice served as a negative control. Differences in glycosylation may cause altered electrophoretic mobility. Bar diagrams show the quantification of the results, data are reported as means $\pm \mathrm{SEM} ; n=5$ Compact, 5 congenic wild-type and $6 \mathrm{BALB} / \mathrm{c}$ mice. $P^{*}<0.05 ; P^{* *}<0.01 ; P^{* * *}<0.001$.

Western blot analysis showed that the level of mature myostatin dimer was the lowest in Compact muscle and highest in BALB/c mice (Figure 18) in accordance with the skeletal muscle weights of the animals. The anti-propeptide antibody could recognize the mutant propeptide, and the expression level of propeptide was proportional to myostatin level when the genotypes were compared; the lowest amount was detected in Compact samples (Figure 18).

\subsubsection{Effects of Compact myostatin mutation and genetic background on signaling}

Myostatin is expressed and secreted predominantly by skeletal muscle; however, as an endocrine factor it, can influence the signal transduction of liver [9]. We could detect the 
mature myostatin; therefore, we investigated myostatin signaling in both skeletal muscle and liver (Figure 19).

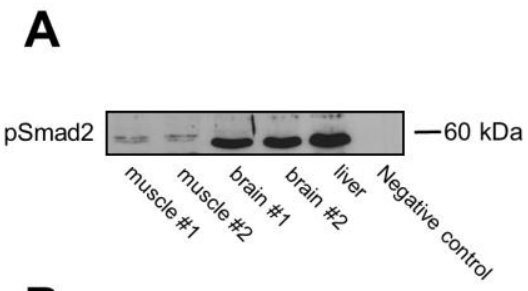

B

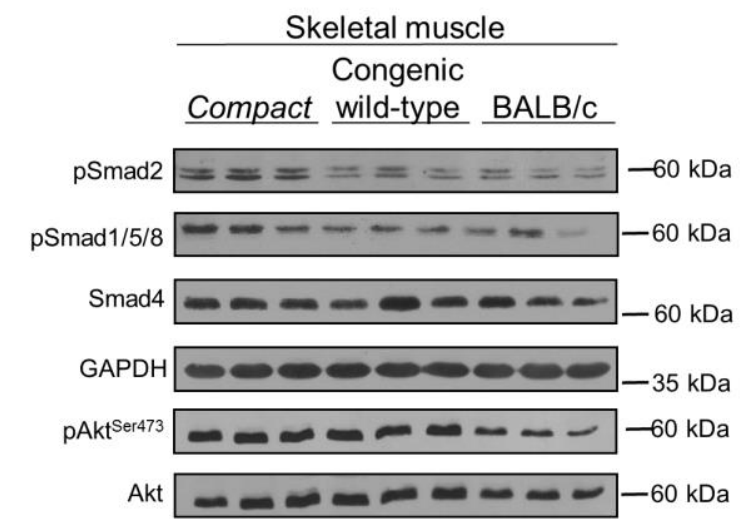

C

Liver

Congenic

Compact wild-type BALB/C

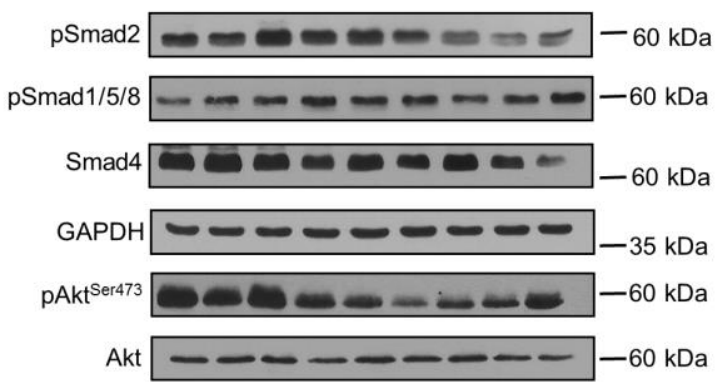

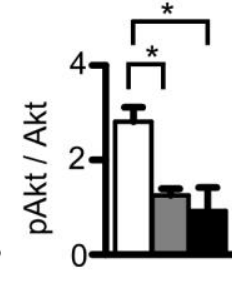

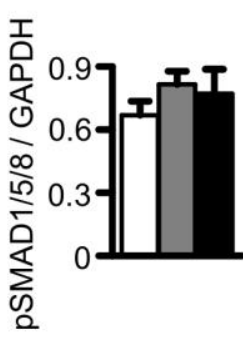

$\square$ Compact $\square$ Congenic wild-type $\square$ BALB/c

Figure 19. Alterations of pSmad2, pSmad1/5/8, and pAkt signaling in the different mice strains. (A) $M$. gastrocnemius homogenates $(50 \mu \mathrm{g}$ protein / lane), mouse brain (40 $\mu \mathrm{g}$ protein / lane) and mouse liver $(60 \mu \mathrm{g}$ protein / lane) samples of Compact mice were subjected to SDS-PAGE and developed by anti-pSmad2 antibody to establish the specificity of the antibody. Brain samples served as a positive control, and the negative control was incubated with only the secondary antibody. Phospho-Smad2 was detected at the predicted molecular weight in either mouse skeletal muscle, liver, or positive control samples. Note the low level of pSmad 2 in skeletal muscle compared with liver and brain tissues. Western blot experiments of Compact, congenic and BALB/c samples indicate the activity of signaling pathways in $M$. gastrocnemius $(B)$ and liver $(C)$ tissues. GAPDH (glyceraldehyde-3-phosphate dehydrogenase) was used as a loading control. Bar diagrams show the quantifications of the results. Data are reported as means $\pm \mathrm{SEM} ; \mathrm{n}=3$ in each group. $P^{*}<0.05 ; P^{* *}<0.01$.

Despite low levels of myostatin protein in Compact skeletal muscle, the level of phospo-Smad2 was the highest when the genotypes were compared (Figure 19B), suggesting the potential role of other TGF- $\beta$ members in Smad2 activation. The specificity of the anti- 
pSmad2 antibody was shown in Figure 19A. Phospho-Smad2 levels of the liver samples were the lowest in BALB/c mice and the highest in Compacts (Figure 19C). Both the Smad2/3 signaling mediated by TGF- $\beta$ family members and the Smad1/5/8 pathway mediated by bone morphogenic proteins (BMPs) converge on the common mediator Smad4. The balance between these competing pathways is required to maintain muscle mass, the BMP-mediated Smad1/5/8 pathway is the fundamental hypertrophic signal in mice, which is dominant over myostatin signaling, and Smad4 deficiency induces muscle atrophy [28]. Furthermore, Smad1/5/8 signaling is important regulator of liver homeostasis [85, 86]. The level of phospho-Smad1/5/8 was significantly higher in muscles of Compacts compared to congenic wild-type samples (Figure 19B). However, we have not found any significant differences in phospho-Smad1/5/8 levels of liver samples between the genotypes (Figure 19C). Furthermore, no differences were observed between either muscular (Figure 19B) or hepatic (Figure 19C) Smad4 levels.

Myostatin was reported to inhibit the PI3K/Akt pathway [22]; therefore, we determined the phosphorylation level of Akt. The phospho-Akt ${ }^{\mathrm{Ser} 473} / \mathrm{Akt}$ ratio of congenic wild-type liver was lower than that of Compact and was comparable with BALB/c values (Figure 19C). In contrast, phospho-Akt ${ }^{\mathrm{Ser} 473} / \mathrm{Akt}$ ratios were higher in Compact and congenic wild-type muscles compared to BALB/c samples in accordance with myostatin levels (Figure 19B).
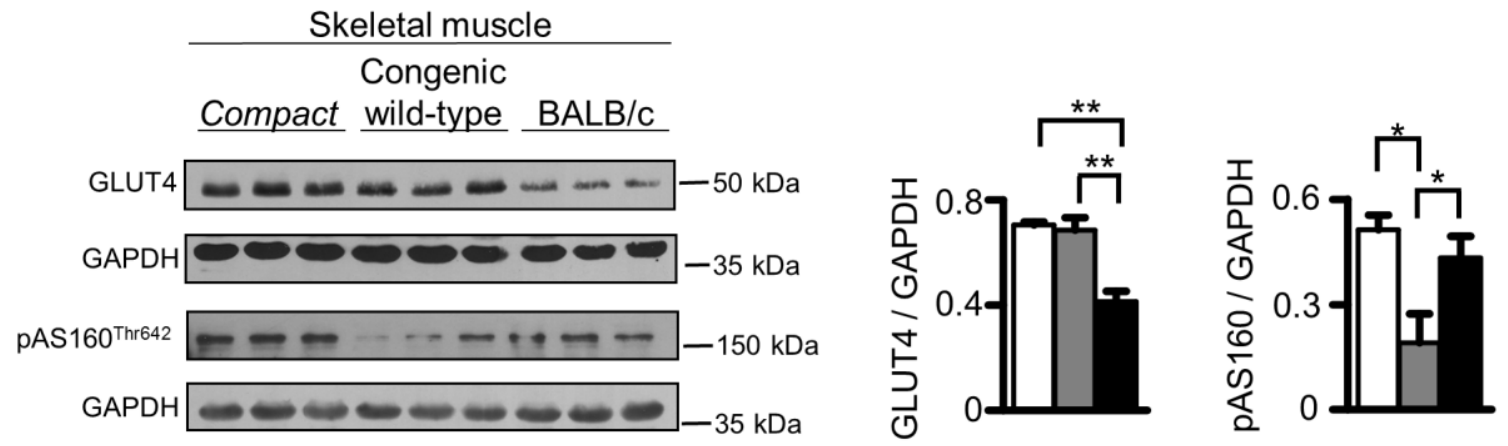

$\square$ Compact $\square$ Congenic wild-type

$\mathrm{BALB} / \mathrm{C}$

Figure 20. Alterations of pAS160 and GLUT4 levels in the different mice strains. Western blot experiments of Compact, congenic and BALB/c samples show the levels of pAS160 and GLUT4 in M. gastrocnemius tissues. GAPDH was used as a loading control. Bar diagrams show the quantifications of the results. Data are reported as means $\pm \mathrm{SEM} ; \mathrm{n}=3$ in each group. $P^{*}<0.05 ; P^{* *}<0.01$. 
AS160 (ㅁkt substrate of $\underline{160} \mathrm{kDa}$ ), a Rab GTPase-activating protein can regulate the translocation of GLUT4 glucose transporter to the plasma membrane of insulin sensitive cells [87]. The level of phospho-AS160 was lower in congenic wild-type muscle samples comparing to those of Compact or BALB/c mice (Figure 20). The GLUT4 expression showed equal levels in Compact and congenic wild-type animals, and the level was lower in BALB/c samples (Figure 20).

\section{DISCUSSION}

Myostatin is a TGF- $\beta$ family member that is expressed and secreted predominantly by skeletal muscle. The function of myostatin appears to be conserved across species, since mutations in the myostatin gene induce bigger muscles in human, mice, cattle, dogs, and sheep [2, 3, 4, 5, 28]. The Compact mutant mice carry a naturally occurring 12-bp non-frame shift deletion in the propeptide region of the precursor myostatin resulting hypermuscularity [3]. The Compact mice arose during a long-term selection program to reach the maximal hypermuscularity [65]. The major gene responsible for the hypermuscular phenotype was mapped on chromosome 1 [69], and after the discovery of myostatin gene [1] the Mstn(Cmpt$d l 1 A b c)$ deletion in the propeptide region of the mouse myostatin was identified as the causative mutation responsible for the Compact phenotype [68]. Due to selection for hypermuscularity, the Compact line, in addition to achieving homozygosity for the $\operatorname{Mstn}(\mathrm{Cmpt}-d l 1 \mathrm{Abc})$ mutation, also accumulated modifier alleles that were involved in the full expression of the phenotype; however, these modifier genes have not been identified yet [68, 69]. The biologically active growth factor domain of myostatin is unaffected by Compact mutation; therefore, the loss of myostatin activity cannot be explained by disruption of the growth factor bioactive domain. However, the Compact mutation can lead to misfolding, or defect in secretion and mistargeting of mature myostatin [3]. The detailed phenotype of the Compact mice has not been investigated earlier, and the molecular consequences of Compact mutation are completely unclear.

To separately study the effect of Compact myostatin mutation and Compact genetic background, we generated a congenic wild-type strain carrying wild-type myostatin in Compact genetic background. We introgressed the wild-type myostatin gene of BALB/c to 
Compact mice to generate the congenic wild-type line and we used BALB/c mice as a wildtype control for the reasons listed by Baán et al. (2013). Briefly, this inbred line was used for mapping the Compact myostatin mutation and the modifier genes [3], and their muscle characteristics are similar to those of C57BL/6 mice. We have found that both the Compact myostatin mutation and the Compact genetic background account for determination of skeletal muscle size. The Compact mice are weighted and hypermuscular compared with both congenic wild-type and BALB/c animals. The Compact mutation resulted in a disproportionate increase in skeletal muscle mass, leading to increased muscle/body weight ratios and decreased internal organ/body weight ratios. Interestingly, the normalized muscle weights of congenic wild-type animals are the smallest despite their increased absolute muscle weights compared to BALB/c mice; therefore, other organs should be involved in accretion of body weight of congenic wild-type animals. Beyond the regulation of muscularity myostatin was earlier shown to influence the size of internal organs. Knocking out of myostatin resulted in decreased weight of fat, liver and kidney as proportional to body weight [35, 36, 37, 38]. The absolute weight of heart increased; however, heart weight/body weight ratio did not change $[35,38]$.

We and others have reported earlier that the Compact mice are hypermuscular [71, 72], and cellularity of the Compact skeletal muscles show increased ratio of glycolytic fibers in rectus femoris, longissimus dorsi [71] and tibialis anterior [72] muscles. Rehfeldt and colleagues (2005) introgressed the Compact mutation into the DUHi mice line. The hypermuscularity of this Compact-DUHi mice was characterized by muscle fiber hyperplasia rather than hypertrophy in fast glycolytic longissimus dorsi and rectus femoris muscles, and by balanced increases in myonuclear proliferation and protein accretion [71]. However, no reports have so far examined the glycogen amount of the Compact muscles and the glycogen content of the individual fiber types. Here we show that the total glycogen amount of the TA, QF and Gastro muscles are markedly greater in Compact than in wild type mice. The phenotype selection on extreme muscle mass in DU6P mice revealed the co-evolution of high glycogen and protein content of the muscle [80]. This finding is in line with our observation on the simultaneous increase of protein and glycogen contents in Compact muscle. The $\mathrm{PI} 3 \mathrm{~K} / A k t$ signaling plays a central role in integrating anabolic and catabolic responses in skeletal muscle influencing muscle mass $[88,89]$. PTEN (Phosphatase and tensin homolog) 
negatively affects the PI3K/Akt pathway; moreover, Sawitzky and colleagues (2012) identified PTEN as a gate keeper molecule in the co-evolutionary regulation of high glycogen and protein content. In order to analyze the glycogen content of the individual muscle fibers the intensity of PAS-staining was measured. However, the value of the average OD of the pixels within a fiber cannot reflect the glycogen content of the whole muscle fiber; the OD value should be multiplied by the fiber size. In accordance with the literature [71] we did not find differences comparing the fiber sizes of the Compact and wild type TA muscles. Furthermore, based on our results, the average glycogen content of the muscle fibers remained unchanged in Compacts compared to wild type mice; therefore, the increased number of fibers can explain the higher total glycogen amount of the Compact TA muscle.

In addition to controlling muscle mass, myostatin also appears to regulate muscle fiber type composition postnatally by regulating the expression of both MyoD and MEF2C (Myocyte Enhancer Factor) genes [33]. Histological analysis revealed increased proportion of type IIB fibers in tibialis anterior, biceps femoris [33] and EDL (extensor digitorum longus) muscles [73] of myostatin knock out mice, or in double-muscled cattle [82, 90]. In accordance with our observations, earlier studies described that the Compact mutation induces a glycolytic shift in the phenotype of skeletal muscle [71, 72]; similarly to the situations evoked by the lack or the dysfunction of myostatin. Rehfeldt and her group reported (2005) that the number of white and intermediate fibers increased in rectus femoris and longissimus dorsi muscles of 70 days old Compact mice, whereas the density of the blood capillaries decreased [71]. Despite the fact that the Compact mice are hypermuscular and their muscles consist of significantly more fibers, the specific force generation decreased in Compact animals [73]. According to our results, the proportion of fast glycolytic fibers in TA muscle of Compact mice increased; however, their individual glycogen content did not.

Regarding the size of the different fiber types we found similar results in both Compact and BALB/c mice: it increased in the rank order IIA < IIX < IIB among the fast fibers. In accordance with our observations, the area of IIB fibers was the biggest in the rectus femoris muscle of Compact-DUHi mice [71]. The average glycogen content of the individual muscle fibers showed similar tendency as the fiber size (i.e. increased in the rank order IIA < IIX < IIB). Thus, the IIB fibers contained the most glycogen in both Compact and BALB/c mice, correlating with the biochemical properties of the fibers. Comparing the superficial and 
deep regions of the TA muscle we found similar results according to the fiber size, intensity of PAS-staining, as well as the glycogen content of the fibers. It is important to note that the measurement of the PAS intensity alone cannot predict clearly the glycogen content of the fibers (i. e. type IIB fibers have lower average PAS intensity but higher fiber size leading to larger glycogen content than that of IIX fibers). Only a few data are available on the glycogen content of the different types of fast fibers, mainly reported in human muscle biopsies. In accordance with our results, type IIA fibers showed more intense PAS-staining than IIB fibers in human biopsy samples [91].

Myostatin was reported to affect glucose metabolism; however, the fed and fasting glucose levels of myostatin knock out male mice were not different from controls [36]. Similarly to these observations, fasting blood glucose levels of Compact mice did not change compared with age-matched congenic wild-type and BALB/c animals. Furthermore, myostatin-null mice have reduced body fat beyond the increased muscle mass and exhibit increased insulin sensitivity [40, 41]. Here, we showed that the Compact mutation increased muscularity and decreased adiposity similarly to myostatin knock out mice, while the genetic background has the opposite effect increasing adiposity and decreasing skeletal muscle mass/body weight ratios. As a consequence of these alterations in body composition the Compact mutation improved whole body glucose tolerance and insulin sensitivity while the genetic background decreased them. The Compact mutation increased the ${ }^{18}$ FDG radiotracer uptake into all investigated organs (white adipose tissue, skeletal muscle and liver). The phosphorylation of AS160 was shown to regulate the translocation of GLUT4 to the plasma membrane [87, 92]. However, the Compact mutation did not influence the amount of GLUT4 in skeletal muscle, but the increased pAS160 level in Compact animals might contribute to the increased glucose uptake by enhancing the GLUT4 translocation to the plasma membrane.

Besides the regulation of insulin sensitivity and glucose tolerance, the Compact genetic background has a role in the regulation of tissue glycogen content. It has opposite impacts on skeletal muscle and liver by decreasing the glycogen level/tissue weight ratio in muscle and increasing it in the liver. These alterations can be at least partially the consequences of decreased glucose uptake into muscle and increased glucose uptake into liver. Consistent with these results reduced muscle glycogen was reported in type 2 diabetes mellitus [79]. The Compact mutation itself did not affect the glycogen level/tissue weight 
ratio either in muscle or liver tissue; however, myostatin treatment was found to reduce the glycogen content of C2C12 myoblasts [46].

Age was shown to affect glucose metabolism; however, no difference was reported in glucose clearance rate comparing 6 and 12 months old BALB/c mice [93]. Consistent with these results the glucose tolerance of BALB/c animals did not change with age (3-4 months vs. 10 months old) in our study. Interestingly, aging reduced glucose tolerance of Compact mice without any significant alteration in sensitivity for exogenous insulin. By testing the enzymatic functions of the liver, despite the smaller liver weight/body weight ratio of Compacts the hepatic gluconeogenesis was comparable with congenic wild-type animals following exogenous pyruvate. The tissue ALT activity of the liver was reduced by Compact myostatin mutation similarly to knocking out of myostatin [37].

The Compact mutation is a non-frameshift deletion in the propeptide; therefore, it raised the possibility that the mature myostatin is present in Compact mice. Here, we have shown that the Compact mutation allows the formation of mature myostatin; however, the amount of myostatin was lower in Compact skeletal muscle in accordance with increased muscle mass. Most of the naturally occurring myostatin mutations lead to the development of an early STOP codon; however, some mutations were shown to be associated with altered proteolysis of promyostatin $[61,94]$ permitting myostatin formation. The specific functions of the propeptides of TGF-superfamily members are largely unknown; however, they can play a role in targeting and inactivation of the biological active C-terminal part, and they have an impact on binding properties to extracellular components. All of these functions of myostatin propeptide can be disturbed by Compact mutation. It was reported that BMP-7 propeptide binds fibrillin-1 [95], BMP-5 propeptide binds fibrillin-1 and -2 [95] and the interaction between myostatin propeptide and perlecan was identified [96]. The interaction of propeptide and myostatin is relevant in vivo, with a majority ( $>70 \%)$ of myostatin in serum bound to its propeptide [29]. The amino acid sequence of GDF-11 (Growth/Differentiation Factor-11) is $90 \%$ homologous to myostatin in the carboxy-terminal mature region of the protein, and like myostatin, GDF-11 can signal through ActRIIB [97], and GDF-11 administration leads to activation of Smad2 signaling [98, 99]. Myostatin propeptide may bind and inhibit GDF-11 as well as myostatin [97]; therefore, the mutant propeptide of Compact mice might disturb not only myostatin but e.g. GDF-11 signaling as well. 
Despite of the low level of myostatin protein in Compact skeletal muscle, the level of phospo-Smad2 was the highest when the genotypes were compared, suggesting the potential role of other TGF- $\beta$ members (e.g. GDF-11) or HGF (Hepatocyte growth factor) in Smad2 activation. HGF is a regulator of satellite cells [100], and transmit signals through Smad2/3. The BMP-mediated Smad1/5/8 signaling is an important regulator of skeletal muscle mass $[27,28]$; hepatocyte proliferation, liver regeneration and function [85, 86]. Comparing the genotypes phospho-Smad1/5/8 levels show the same pattern as tissue weight/body weight ratios in both skeletal muscle and liver suggesting the impact of Smad1/5/8 signaling on regulation of skeletal muscle and liver size. The BMP inhibitor chordin was proposed as a modifier gene in Compact mice [69], that can influence the activity of BMP pathway by binding and modulating the effect of BMPs [101]. Interestingly, the elevated phosphoSmad1/5/8 levels can derive not only from BMPs, since TGF- $\beta$ was reported to stimulate the phosphorylation of Smad1/5 through a non-canonical mechanism [102].

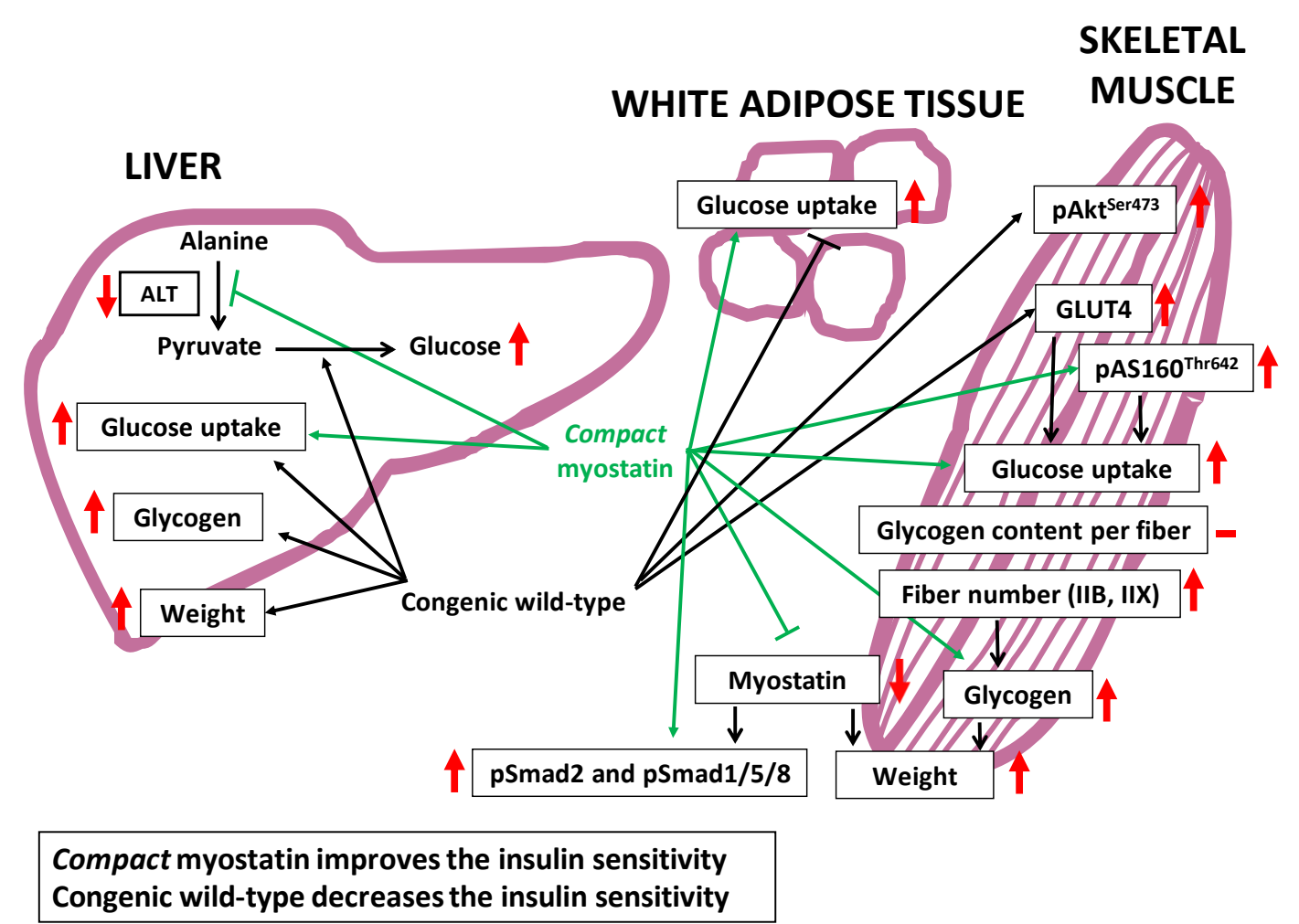

Figure 21. The major conclusions of the thesis. The metabolic effects of the Compact myostatin and the genetic background can compensate for each other. 
The Compact mice represent a complex system consisting of a natural mutation in the propeptide of promyostatin and additional modifier genes. The Compact mice show several similarities compared to myostatin knock out animals; however, numerous alterations exist due to the redundant function of propeptide and the presence of the specific Compact genetic background. Myostatin propeptide may bind and inhibit GDF-11 as well [97]; therefore, the effect of Compact propeptide cannot be restricted to myostatin signaling. Our analysis has shown that the modifier genes of the genetic background can strengthen the effect of Compact myostatin mutation, or they can compensate for each other (Figure 21). Further analysis of the biological effect of Compact mutation and the identification of modifier genes may provide a route to additional upstream and downstream factors involved in the regulation of skeletal muscle size and metabolism.

\section{CONCLUSIONS}

In the present thesis we investigated the effects of the Compact mutation Mstn(Cmpt$d l 1 A b c$ ) on the morphology, metabolism, and signaling. We used mice strains carrying mutant or wild-type myostatin alleles with Compact genetic background and non-mutant myostatin with wild-type background. By these strains we studied the effect of the of the Mstn(Cmpt$d l 1 A b c)$ mutation or the Compact genetic background.

The major findings of the thesis are as follows:

1. We have found that both the Compact mutant myostatin and the genetic background increase the skeletal muscle mass.

2. The increased glycogen and protein content of the Compact muscles may lead to increased skeletal musle mass.

3. Although the average glycogen content of the individual fibers kept unchanged in Compact muscle, the number of IIB and IIX fibers increased leading to higher glycogen content of the muscle. 
4. Both the Compact myostatin and the genetic background exhibit systemic, metabolic effects.

5. The Compact mutation does not influence the absolute weight of the internal organs (heart, kidney, liver, abdominal fat) despite the increased absolute weight of the skeletal muscle .

6. The genetic background increases the absolute weight and glycogen content of the liver; furthermore, it increases the glucose uptake and hepatic gluconeogenesis.

7. The Compact myostatin decreases adipositiy, improves insulin sensitivity, enhances the glucose uptake of the skeletal muscle and white adipose tissue, whereas the genetic background has opposite effects.

8. The Compact mutation does not prevent the formation of myostatin; however, it decreases the amount of mature myostatin leading to altered activation of Smad2, Smad1/5/8 and Akt.

9. The increased level of phospho-AS160 $60^{\text {Thr642 }}$ in Compact samples myostatin may lead to increased glucose uptake of the skeletal muscle. 


\section{ACKNOWLEDGEMENTS}

Firstly, I would like to give my special thanks for Professor László Dux for providing possibility for my work and encouraged me to join the Department of Biochemistry.

I would like to give my greatly thanks for Anikó Keller-Pintér for supervising and the helpful advices for my work during on graduate as well as $\mathrm{PhD}$ years. She greatly introduced me to the scientific work and analytical thinking.

I would like to give my thanks for my co-authors. Especially, György Trencsényi for the PET/MRI measurement and Géza Müller for the advices in mice crossing and Ferenc Deák for his critical reading and help in the manuscript drifting.

Furthermore, I would like give my thanks to Kitti Szabó for her help during the experiments. I would like to give my greatly thanks for Lászlóné Csontos for her excellent assistance during my experimental work and Zita Makráné Felhő and Istvánné Balásházy for their assistance and help during my $\mathrm{PhD}$ years.

I would like my thanks to all of my former colleagues in the Department of Biochemistry.

Last but not least I would like give my greatly thanks for my family to supported me during my work.

This research was supported by the European Union and the State of Hungary and cofinanced by the European Social Fund in the framework of the TÁMOP 4.2.4. A/2-11-1-2012-0001 "National Excellence Program", the UNKP-17-4 New National Excellence Program of the Ministry of Human Capacities, GINOP-2.3.2-15-2016-00006, and GINOP-2.3.2-15-201600040 grants of the National Research, Development and Innovation Office. 


\section{REFERENCES}

1. McPherron AC, Lawler AM, Lee SJ. Regulation of skeletal muscle mass in mice by a new TGFbeta superfamily member. Nature 387: 83-90, 1997.

2. Schuelke M, Wagner KR, Stolz LE, Hübner C, Riebel T, Kömen W, Braun T, Tobin JF, Lee SJ. Myostatin mutation associated with gross muscle hypertrophy in a child. $N$ Engl J Med 350: 2682-2688, 2004.

3. Szabó G, Dallmann G, Müller G, Patthy L, Soller M, Varga L. A deletion in the myostatin gene causes the compact (Cmpt) hypermuscular mutation in mice. Mamm Genome 9: 671-672, 1998.

4. Kambadur R, Sharma M, Smith TP, Bass JJ. Mutations in myostatin (GDF8) in doublemuscled Belgian Blue and Piedmontese cattle. Genome Res 7: 910-916, 1997.

5. Clop A, Marcq F, Takeda H, Pirottin D, Tordoir X, Bibé B, Bouix J, Caiment F, Elsen JM, Eychenne F, Larzul C, Laville E, Meish F, Milenkovic D, Tobin J, Charlier C, Georges M. A mutation creating a potential illegitimate microRNA target site in the myostatin gene affects muscularity in sheep. Nat Genet 38: 813-818, 2006.

6. Langley B, Thomas M, Bishop A, Sharma M, Gilmour S, Kambadur R. Myostatin inhibits myoblast differentiation by down-regulating MyoD expression. J Biol Chem 277: 49831-49840, 2002.

7. Thomas M, Langley B, Berry C, Sharma M, Kirk S, Bass J, Kambadur R. Myostatin, a negative regulator of muscle growth, functions by inhibiting myoblast proliferation. $J$ Biol Chem 275: 40235-40243, 2000.

8. McCroskery S, Thomas M, Maxwell L, Sharma M, Kambadur R. Myostatin negatively regulates satellite cell activation and self-renewal. J Cell Biol 162: 1135-1147, 2003. 
9. Williams NG, Interlichia JP, Jackson MF, Hwang D, Cohen P, Rodgers BD. Endocrine actions of myostatin: systemic regulation of the IGF and IGF binding protein axis. Endocrinology 152: 172-180, 2011.

10. Sharma M. , Kambadur R, Matthews KG, Somers WG, Devlin GP, Conaglen JV, Fowke PJ, Bass JJ. Myostatin, a transforming growth factor-beta superfamily member, is expressed in heart muscle and is upregulated in cardiomyocytes after infarct. J Cell Physiol 180: 1-9, 1999.

11. Jiao J, Yuan T, Zhou Y, Xie W, Zhao Y, Zhao J, Ouyang H, Pang D. Analysis of myostatin and its related factors in various porcine tissues. J Anim Sci 89: 3099-3106, 2011.

12. Ji S, Losinski RL, Cornelius SG, Frank GR, Willis GM, Gerrard DE, Depreux FF, Spurlock ME. Myostatin expression in porcine tissues: tissue specificity and developmental and postnatal regulation. Am J Physiol 275: R1265-R1273, 1998.

13. Lee SJ, McPherron AC. Regulation of myostatin activity and muscle growth. Proc Natl Acad Sci USA 98: 9306-9311, 2001.

14. Jin HJ, Dunn MA, Borthakur D, Kim YS. Refolding and purification of unprocessed porcine myostatin expressed in Escherichia coli. Protein Expr Purif 35: 1-10, 2004.

15. Thies RS, Chen T, Davies MV, Tomkinson KN, Pearson AA, Shakey QA, Wolfman NM. GDF-8 propeptide binds to GDF-8 and antagonizes biological activity by inhibiting GDF-8 binding. Growth Factors 18: 251-259, 2001.

16. Anderson SB, Goldberg AL, Whitman M. Identification of a novel pool of extracellular pro-myostatin in skeletal muscle. J Biol Chem 283: 7027-7035, 2008.

17. Wolfman NM, McPherron AC, Pappano WN, Davies MV, Song K, Tomkinson KN, Wright JF, Zhao L, Sebald SM, Greenspan DS, Lee SJ. Activation of latent myostatin by 
the BMP-1/tolloid family of metalloproteinases. Proc Natl Acad Sci USA 100: 15842-15846, 2003.

18. Massagué J. TGF-beta signal transduction. Annu Rev Biochem 67: 753-791, 1998.

19. Zhu X, Topouzis S, Liang LF, Stotish RL. Myostatin signaling through Smad2, Smad3 and Smad4 is regulated by the inhibitory Smad7 by a negative feedback mechanism. Cytokine 26: 262-272, 2004.

20. Jayaraman L, Massague J. Distinct oligomeric states of SMAD proteins in the transforming growth factor-beta pathway. J Biol Chem 275: 40710-40717, 2000.

21. Kollias HD, McDermott JC. Transforming growth factor-beta and myostatin signaling in skeletal muscle. J Appl Physiol 104: 579-587, 2008.

22. Yang W, Zhang Y, Li Y, Wu Z, Zhu D. Myostatin induces cyclin D1 degradation to cause cell cycle arrest through a phosphatidylinositol 3-kinase/AKT/GSK-3 beta pathway and is antagonized by insulin-like growth factor 1. J Biol Chem 282: 3799-3808, 2007.

23. Trendelenburg AU, Meyer A, Rohner D, Boyle J, Hatakeyama S, Glass DJ. Myostatin reduces Akt/TORC1/p70S6K signaling, inhibiting myoblast differentiation and myotube size. Am J Physiol Cell Physiol 296: C1258-C1270, 2009.

24. Cohen S, Brault JJ, Gygi SP, Glass DJ, Valenzuela DM, Gartner C, Latres E, Goldberg AL. During muscle atrophy, thick, but not thin, filament components are degraded by MuRF1-dependent ubiquitylation. J Cell Biol 185: 1083-1095, 2009.

25. Zhao TJ, Yan YB, Liu Y, Zhou HM. The generation of the oxidized form of creatine kinase is a negative regulation on muscle creatine kinase. J Biol Chem 282: 12022-12029, 2007. 
26. Allen DL, Unterman TG. Regulation of myostatin expression and myoblast differentiation by FoxO and SMAD transcription factors. Am J Physiol Cell Physiol 292: C188-C199, 2007.

27. Winbanks CE, Chen JL, Qian H, Liu Y, Bernardo BC, Beyer C, Watt KI, Thomson RE, Connor T, Turner BJ, McMullen JR, Larsson L, McGee SL, Harrison CA, Gregorevic P. The bone morphogenetic protein axis is a positive regulator of skeletal muscle mass. J Cell Biol 203: 345-357, 2013.

28. Sartori R, Schirwis E, Blaauw B, Bortolanza S, Zhao J, Enzo E, Stantzou A, Mouisel E, Toniolo L, Ferry A, Stricker S, Goldberg AL, Dupont S, Piccolo S, Amthor H, Sandri M. BMP signaling controls muscle mass. Nat Genet 45: 1309-1318, 2013.

29. Hill JJ, Davies MV, Pearson AA, Wang JH, Hewick RM, Wolfman NM, Qiu Y. The myostatin propeptide and the follistatin-related gene are inhibitory binding proteins of myostatin in normal serum. J Biol Chem 277: 40735-40741, 2002.

30. Hill JJ, Qiu Y, Hewick RM, Wolfman NM. Regulation of myostatin in vivo by growth and differentiation factor-associated serum protein-1: a novel protein with protease inhibitor and follistatin domains. Mol Endocrinol 17: 1144-1154, 2003.

31. Lee YS and Se-Jin Lee SJ. Regulation of GDF-11 and myostatin activity by GASP-1 and GASP-2. Proc Natl Acad Sci USA 110: E3713-E3722, 2013.

32. Miura T, Kishioka Y, Wakamatsu J, Hattori A, Hennebry A, Berry CJ, Sharma M, Kambadur R, Nishimura T. Decorin binds myostatin and modulates its activity to muscle cells. Biochem Biophys Res Commun 340: 675-680, 2006.

33. Hennebry A, Berry C, Siriett V, O'Callaghan P, Chau L, Watson T, Sharma M, Kambadur R. Myostatin regulates fiber-type composition of skeletal muscle by regulating MEF-2 and MyoD gene expression. Am J Physiol Cell Physiol 296: C525-C534, 2009. 
34. Mendias CL, Kayupov E, Bradley JR, Brooks SV, Claflin DR. Decreased specific force and power production of muscle fibers from myostatin-deficient mice are associated with a suppression of protein degradation. Appl Physiol 111: 185-191, 2011.

35. Lin J, Arnold HB, Della-Fera MA, Azain MJ, Hartzell DL, Baile CA. Myostatin knockout in mice increases myogenesis and decreases adipogenesis. Biochem Biophys Res Commun 291: 701-706, 2002.

36. McPherron AC, Lee SJ. Suppression of body fat accumulation in myostatin-deficient mice. J Clin Invest 109: 595-601, 2002.

37. Huang J, Glauber M, Qiu Z, Gazit V, Dietzen DJ, Rudnick DA. The influence of skeletal muscle on the regulation of liver:body mass and liver regeneration. Am J Pathol 180: 575-582, 2012.

38. Jackson MF, Luong D, Vang DD, Garikipati DK, Stanton JB, Nelson OL, Rodgers BD. The aging myostatin null phenotype: reduced adiposity, cardiac hypertrophy, enhanced cardiac stress response, and sexual dimorphism. J Endocrinol 213: 263-275, 2012.

39. Guo W, Wong S, Bhasin S. AAV-mediated administration of myostatin pro-peptide mutant in adult Ldlr null mice reduces diet-induced hepatosteatosis and arteriosclerosis. PLoS One 8: e71017, 2013.

40. Guo T, Jou W, Chanturiya T, Portas J, Gavrilova O, McPherron AC. Myostatin inhibition in muscle, but not adipose tissue, decreases fat mass and improves insulin sensitivity. PLoS One 4: e4937, 2009.

41. Zhang C, McFarlane C, Lokireddy S, Bonala S, Ge X, Masuda S, Gluckman PD, Sharma M, Kambadur R. Myostatin-deficient mice exhibit reduced insulin resistance through activating the AMP-activated protein kinase signalling pathway. Diabetologia 54: 1491-1501, 2011. 
42. Morissette MR, Stricker JC, Rosenberg MA, Buranasombati C, Levitan EB, Mittleman MA, Rosenzweig A. Effects of myostatin deletion in aging mice. Aging Cell 8: 573-583, 2009.

43. Zhao B, Wall RJ, Yang J. Transgenic expression of myostatin propeptide prevents dietinduced obesity and insulin resistance. Biochem Biophys Res Commun 337: 248-255, 2005.

44. Brandt C, Hansen RH, Hansen JB, Olsen CH, Galle P, Mandrup-Poulsen T, Gehl J, Pedersen BK, Hojman P. Over-expression of Follistatin-like 3 attenuates fat accumulation and improves insulin sensitivity in mice. Metabolism 64: 283-295, 2015.

45. Guo T, Bond ND, Jou W, Gavrilova O, Portas J, McPherron AC. Myostatin inhibition prevents diabetes and hyperphagia in a mouse model of lipodystrophy. Diabetes 61: 24142423, 2012.

46. Chen Y, Ye J, Cao L, Zhang Y, Xia W, Zhu D. Myostatin regulates glucose metabolism via the AMP-activated protein kinase pathway in skeletal muscle cells. Int J Biochem Cell Biol 42: 2072-2081, 2010.

47. Yarasheski KE, Bhasin S, Sinha-Hikim I, Pak-Loduca J, Gonzalez-Cadavid NF. Serum myostatin-immunoreactive protein is increased in 60-92 year old women and men with muscle wasting. J Nutr Health Aging 6: 343-348, 2002.

48. Ju CR, Chen RC. Serum myostatin levels and skeletal muscle wasting in chronic obstructive pulmonary disease. Respir Med 106: 102-108, 2012.

49. Gonzalez-Cadavid NF, Taylor WE, Yarasheski K, Sinha-Hikim I, Ma K, Ezzat S, Shen R, Lalani R, Asa S, Mamita M, Nair G, Arver S, Bhasin S. Organization of the human myostatin gene and expression in healthy men and HIV-infected men with muscle wasting. Proc Natl Acad Sci USA 95: 14938-14943, 1998. 
50. Yano S, Nagai A, Isomura M, Yamasaki M, Kijima T, Takeda M, Hamano T, Nabika T. Relationship between Blood Myostatin Levels and Kidney Function: Shimane CoHRE Study. PLoS One 10: e0141035, 2015.

51. Costelli P, Muscaritoli M, Bonetto A, Penna F, Reffo P, Bossola M, Bonelli G, Doglietto GB, Baccino FM, Rossi Fanelli F. Muscle myostatin signalling is enhanced in experimental cancer cachexia. Eur J Clin Invest 38: 531-538, 2008.

52. Reardon KA, Davis J, Kapsa RM, Choong P, Byrne E. Myostatin, insulin-like growth factor-1, and leukemia inhibitory factor mRNAs are upregulated in chronic human disuse muscle atrophy. Muscle Nerve 24: 893-899, 2001.

53. Anker SD, Swan JW, Volterrani M, Chua TP, Clark AL, Poole-Wilson PA, Coats AJ. The influence of muscle mass, strength, fatigability and blood flow on exercise capacity in cachectic and non-cachectic patients with chronic heart failure. Eur Heart J 18: 259-269, 1997.

54. Strassburg S, Springer J, Anker SD. Muscle wasting in cardiac cachexia. Int J Biochem Cell Biol 37: 1938-1947, 2005.

55. Furihata T, Kinugawa S, Fukushima A, Takada S, Homma T, Masaki Y, Abe T, Yokota T, Oba K, Okita K, Tsutsui H. Serum myostatin levels are independently associated with skeletal muscle wasting in patients with heart failure. Int J Cardiol 220: 483-487, 2016.

56. George I, Bish LT, Kamalakkannan G, Petrilli CM, Oz MC, Naka Y, Sweeney HL, Maybaum S. Myostatin activation in patients with advanced heart failure and after mechanical unloading. Eur J Heart Fail 12: 444-453, 2010.

57. Hittel DS, Berggren JR, Shearer J, Boyle K, Houmard JA. Increased secretion and expression of myostatin in skeletal muscle from extremely obese women. Diabetes 58: 30-38, 2009. 
58. Han DS, Chu-Su Y, Chiang CK, Tseng FY, Tseng PH, Chen CL, Wu KD, Yang WS. Serum myostatin is reduced in individuals with metabolic syndrome. PLoS One 9: e108230, 2014.

59. Bogdanovich S, Krag TO, Barton ER, Morris LD, Whittemore LA, Ahima RS, Khurana TS. Functional improvement of dystrophic muscle by myostatin blockade. Nature 420: 418-421, 2002.

60. Wagner KR, Fleckenstein JL, Amato AA, Barohn RJ, Bushby K, Escolar DM, Flanigan KM, Pestronk A, Tawil R, Wolfe GI, Eagle M, Florence JM, King WM, Pandya S, Straub V, Juneau P, Meyers K, Csimma C, Araujo T, Allen R, Parsons SA, Wozney JM, Lavallie ER, Mendell JR. A phase I/IItrial of MYO-029 in adult subjects with muscular dystrophy. Ann Neurol 63: 561-571, 2008.

61. Berry C, Thomas M, Langley B, Sharma M, Kambadur R. Single cysteine to tyrosine transition inactivates the growth inhibitory function of Piedmontese myostatin. Am J Physiol Cell Physiol 283: C135-C141, 2002.

62. Grobet L, Poncelet D, Royo LJ, Brouwers B, Pirottin D, Michaux C, Ménissier F, Zanotti M, Dunner S, Georges M. Molecular definition of an allelic series of mutations disrupting the myostatin function and causing double-muscling in cattle. Mamm Genome 9: 210-213, 1998.

63. Mosher DS, Quignon P, Bustamante CD, Sutter NB, Mellersh CS, Parker HG, Ostrander EA. A mutation in the myostatin gene increases muscle mass and enhances racing performance in heterozygote dogs. PLoS Genet 3: e79, 2007.

64. Johnson PL, McEwan JC, Dodds KG, Purchas RW, Blair HT. Meat quality traits were unaffected by a quantitative trait locus affecting leg composition traits in Texel sheep. J Anim Sci 83: 2729-2735, 2005. 
65. Bünger L, Laidlaw A, Bulfield G, Eisen EJ, Medrano JF, Bradford GE, Pirchner F, Renne U, Schlote W, Hill WG. Inbred lines of mice derived from long-term on growth selected lines: unique resources for mapping growth genes. Mamm Genome 12: 678-686, 2001.

66. Bünger L, Ott G, Varga L, Schlote W, Rehfeldt C, Williams JL, Hill WG. Marker assisted introgression of the Compact mutant myostatin allele: MstnCmpt-dl1 Abc into a mouse line with extreme growth-effects on body composition and muscularity. Genet Res 84: 161-173, 2004.

67. Varga L, Szabó G, Darvasi A, Müller G, Sass M, Soller M. Inheritance and mapping of Compact (Cmpt), a new mutation causing hypermuscularity in mice. Genetics 147: 755-764, 1997.

68. Varga L, Müller G, Szabó G, Pinke O, Korom E, Kovács B, Patthy L, Soller M. Mapping modifiers affecting muscularity of the myostatin mutant (Mstn(Cmpt-dl1Abc)) compact mouse. Genetics 165: 257-267, 2003.

69. Varga L, Pinke O, Müller G, Kovács B, Korom E, Szabó G, Soller M. Mapping a syntenic modifier on mouse chromosome 1 influencing the expressivity of the compact phenotype in the myostatin mutant (MstnCmpt-dl1Abc) compact mouse. Genetics 169: 489493, 2005.

70. Abreu JG, Ketpura NI, Reversade B, De Robertis EM. Connective-tissue growth factor (CTGF) modulates cell signalling by BMP and TGF-beta. Nat Cell Biol 4: 599-604, 2002.

71. Rehfeldt C, Ott G, Gerrard DE, Varga L, Schlote W, Williams JL, Renne U, Bünger

L. Effects of the compact mutant myostatin allele Mstn (Cmpt-dl1Abc) introgressed into a high growth mouse line on skeletal muscle cellularity. J Muscle Res Cell Motil 26: 103-112, 2005. 
72. Baán JA, Kocsis T, Keller-Pintér A, Müller G, Zádor E, Dux L, Mendler L. The compact mutation of myostatin causes a glycolytic shift in the phenotype of fast skeletal muscles. J Histochem Cytochem 61: 889-900, 2013.

73. Amthor H, Macharia R, Navarrete R, Schuelke M, Brown SC, Otto A, Voit T, Muntoni F, Vrbóva G, Partridge T, Zammit P, Bunger L, Patel K. Lack of myostatin results in excessive muscle growth but impaired force generation. Proc Natl Acad Sci U S A 104: 1835-1840, 2007.

74. Bodnár D, Geyer N, Ruzsnavszky O, Oláh T, Hegyi B, Sztretye M, Fodor J, Dienes B, Balogh Á, Papp Z, Szabó L, Müller G, Csernoch L, Szentesi P. Hypermuscular mice with mutation in the myostatin gene display altered calcium signalling. J Physiol 592: 1353$1365,2014$.

75. Weniger JH, Horst P, Steinhauf D, Major F, Wolf M, Tawfik ES. Model experiments on selection for endurance and its relation to growth. Part I. Introduction, methods and preliminary investigations on the basic population. Journal of Animal Breeding and GeneticsZeitschrift für Tierzüchtung und Züchtungsbiologie 91: 265-270, 1974.

76. Smerdu V, Soukup T. Demonstration of myosin heavy chain isoforms in rat and humans: the specificity of seven available monoclonal antibodies used in immunohistochemical and immunoblotting methods. Eur Journal Histochem 52: 179-190, 2008.

77. Schiaffino S, Gorza L, Sartore S, Saggin L, Ausoni S, Vianello M, Gundersen K, Lømo T. Three myosin heavy chain isoforms in type 2 skeletal muscle fibers. J Muscle Res Cell Motil 10: 197-205, 1989.

78. Ishihara A, Hirofuji C, Nakatani T, Itoh K, Itoh M, Katsuta S. Effects of running exercise with increasing loads on TA muscle fibres in mice. Exp Physiol 87: 113-116, 2002. 
79. He J, Kelley DE. Muscle glycogen content in type 2 diabetes mellitus. Am J Physiol Endocrinol Metab 287: E1002-E1007, 2004.

80. Sawitzky M, Zeissler A, Langhammer M, Bielohuby M, Stock P, Hammon HM, Görs S, Metges CC, Stoehr BJ, Bidlingmaier M, Fromm-Dornieden C, Baumgartner BG, Christ B, Brenig B, Binder G, Metzger F, Renne U, Hoeflich A. Phenotype selection reveals coevolution of muscle glycogen and protein and PTEN as a gate keeper for the accretion of muscle mass in adult female mice. Plos One 7: e39711, 2012.

81. Carlson CJ, Booth FW, Gordon SE. Skeletal muscle myostatin mRNA expression is fiber-type specific and increases during hindlimb unloading. Am J Physiol Regul Integr Comp Physiol 277: R601-R606, 1999.

82. Matsakas A, Mouisel E, Amthor H, Patel K. Myostatin knockout mice increase oxidative muscle phenotype as an adaptive response to exercise. J Muscle Res Cell Motil 31: 111-125, 2010.

83. Ayala JE, Samuel VT, Morton GJ, Obici S, Croniger CM, Shulman GI, Wasserman DH, McGuinness OP; NIH Mouse Metabolic Phenotyping Center Consortium. Standard operating procedures for describing and performing metabolic tests of glucose homeostasis in mice. Dis Model Mech 3: 525-534, 2010.

84. Mathew D, Zhou P, Pywell CM, van der Veen DR, Shao J, Xi Y, Bonar NA, Hummel AD, Chapman S, Leevy WM, Duffield GE. Ablation of the ID2 gene results in altered circadian feeding behavior, and sex-specific enhancement of insulin sensitivity and elevated glucose uptake in skeletal muscle and brown adipose tissue. PLoS One 8: e73064, 2013.

85. Do N, Zhao R, Ray K, Ho K, Dib M, Ren X, Kuzontkoski P, Terwilliger E, Karp SJ. BMP4 is a novel paracrine inhibitor of liver regeneration. Am J Physiol Gastrointest Liver Physiol 303: G1220-G1227, 2012. 
86. Sugimoto H, Yang C, LeBleu VS, Soubasakos MA, Giraldo M, Zeisberg M, Kalluri

R. BMP-7 functions as a novel hormone to facilitate liver regeneration. FASEB $J$ 21: 256264, 2007.

87. Klip A, Sun Y, Chiu TT, Foley KP. Signal transduction meets vesicle traffic: the software and hardware of GLUT4 translocation. Am J Physiol Cell Physiol 306: C879-C886, 2014.

88. Chelh I, Meunier B, Picard B, Reecy MJ, Chevalier C, Hocquette JF, Cassar-Malek I. Molecular profiles of Quadriceps muscle in myostatin-null mice reveal PI3K and apoptotic pathways as myostatin targets. BMC Genomics 10: 196, 2009.

89. Wu M, Falasca M, Blough ER. Akt/protein kinase B in skeletal muscle physiology and pathology. J Cell Physiol 226: 29-36, 2011.

90. Holmes JH, Ashmore CR. A histochemical study of development of muscle fibre type and size in normal and double muscled cattle. Growth 36: 351-372, 1972.

91. Dubowitz VC, Sewry A, Oldfors A. Normal Muscle. In: Muscle Biopsy A Practical Approach. 4th ed. Dubowitz, VC, Sewry A, Oldfors A, editors. Saunders Elsevier; 2013. p. $28-55$.

92. Sakamoto K, Holman GD. Emerging role for AS160/TBC1D4 and TBC1D1 in the regulation of GLUT4 traffic. Am J Physiol Endocrinol Metab 295: E29-E37, 2008.

93. Nankervis SA, Mitchell JM, Charchar FJ, McGlynn MA, Lewandowski PA. Consumption of a low glycaemic index diet in late life extends lifespan of Balb/c mice with differential effects on DNA damage. Longev Healthspan 2: 4, 2013.

94. Szláma G, Trexler M, Buday L, Patthy L. K153R polymorphism in myostatin gene increases the rate of promyostatin activation by furin. FEBS Lett 589: 295-301, 2015. 
95. Sengle G, Charbonneau NL, Ono RN, Sasaki T, Alvarez J, Keene DR, Bächinger HP, Sakai LY. Targeting of bone morphogenetic protein growth factor complexes to fibrillin. $J$ Biol Chem 283: 13874-13888, 2008.

96. Sengle G, Ono RN, Sasaki T, Sakai LY. Prodomains of transforming growth factor beta (TGFbeta) superfamily members specify different functions: extracellular matrix interactions and growth factor bioavailability. J Biol Chem 286: 5087-5099, 2011.

97. McPherron AC. Metabolic functions of myostatin and GDF11. Immunol Endocr Metab Agents Med Chem 10: 217-231, 2010.

98. Egerman MA, Cadena SM, Gilbert JA, Meyer A, Nelson HN, Swalley SE, Mallozzi C, Jacobi C, Jennings LL, Clay I, Laurent G, Ma S, Brachat S, Lach-Trifilieff E, Shavlakadze T, Trendelenburg AU, Brack AS, Glass DJ. GDF11 Increases with Age and Inhibits Skeletal Muscle Regeneration. Cell Metab 22: 164-174, 2015.

99. Poggioli T, Vujic A, Yang P, Macias-Trevino C, Uygur AN, Loffredo FS, Pancoast JR, Cho M, Goldstein J, Tandias RM, Gonzalez E, Walker RG, Thompson TB, Wagers AJ, Fong YW, Lee RT. Circulating growth differentiation factor 11/8 levels decline with Age. Circ Res 118: 29-37, 2016.

100. Tatsumi R, Anderson JE, Nevoret CJ, Halevy O, Allen RE. HGF/SF is present in normal adult skeletal muscle and is capable of activating satellite cells. Dev Biol 194: 114$128,1998$.

101. Zakin L, De Robertis EM. Extracellular regulation of BMP signalling. Curr Biol 20: R89-R92, 2010.

102. Liu IM, Schilling SH, Knouse KA, Choy L, Derynck R, Wang XF. TGFbetastimulated Smad1/5 phosphorylation requires the ALK5 L45 loop and mediates the promigratory TGFbeta switch. EMBO J 28: 88-89, 2009. 
13. ANNEX 


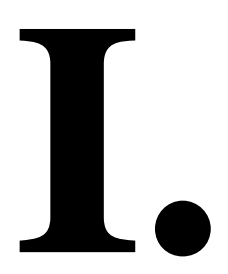




\section{Skeletal muscle cellularity and glycogen distribution in the hypermuscular Compact mice}

\author{
T. Kocsis, ${ }^{1}$ J. Baán, ${ }^{1}$ G. Müller, ${ }^{2}$ \\ L. Mendler, ${ }^{1}$ L. Dux, ${ }^{1}$ A. Keller-Pintér ${ }^{1}$ \\ 'Department of Biochemistry, Faculty \\ of General Medicine, University of Szeged \\ ${ }^{2}$ Egis Pharmaceuticals, Budapest, \\ Hungary
}

\section{Abstract}

The TGF-beta member myostatin acts as a negative regulator of skeletal muscle mass. The Compact mice were selected for high protein content and hypermuscularity, and carry a naturally occurring 12-bp deletion in the propeptide region of the myostatin precursor. We aimed to investigate the cellular characteristics and the glycogen distribution of the Compact tibialis anterior (TA) muscle by quantitative histochemistry and spectrophotometry. We have found that the deficiency in myostatin resulted in significantly increased weight of the investigated hindlimb muscles compared to wild type. Although the average glycogen content of the individual fibers kept unchanged, the total amount of glycogen in the Compact TA muscle increased two-fold, which can be explained by the presence of more fibers in Compact compared to wild type muscle. Moreover, the ratio of the most glycolytic IIB fibers significantly increased in the Compact TA muscle, of which glycogen content was the highest among the fast fibers. In summary, myostatin deficiency caused elevated amount of glycogen in the TA muscle but did not increase the glycogen content of the individual fibers despite the marked glycolytic shift observed in Compact mice.

\section{Introduction}

Myostatin (growth/differentiation factor-8, GDF-8), a member of the TGF-beta superfamily is a potent negative regulator of skeletal muscle growth. ${ }^{1}$ Knocking out of myostatin or naturally occurring mutations, e.g., in human, mice, cattle, dog and porcine ${ }^{2-6}$ lead to large and widespread increase of the skeletal muscle mass. Knockout mice show marked hypermuscularity due to both hyperplasia (increased fiber number) and hypertrophy (increased thickness of the fibers), ${ }_{1}^{1}$ whereas Belgian Blue double-muscled (DM) cattle contain almost double number of muscle fibers due to myostatin mutation. ${ }^{7,8}$ Muscle metabolism in DM cattle or knockout mice shifted into the glycolytic toward exhibiting an increased proportion of fast glycolytic white fibers. -10 $^{8-10}$

The Compact mice arose during a long-term selection program to reach the maximal protein accretion and hypermuscularity. ${ }^{11}$ Genetic analysis of the Hungarian subpopulation of the Compact line identified a 12-bp deletion in the prodomain region of the precursor myostatin; $; 2$ therefore, the biologically active growth factor part is intact. However, additional modifier genes should be present to determine the full expressivity of the phenotype. ${ }^{13,14}$ The precise biological, biochemical effect of the Compact mutation is poorly understood; the mutant propeptide region may play a role in the proper folding, secretion and targeting of mature myostatin. ${ }^{12}$ Hypermuscularity caused by Compact mutation results from muscle fiber hyperplasia rather than hypertrophy, and muscle metabolism shifted towards glycolytic direction. ${ }^{15,16}$ We hypothesized that not only the increased protein amount but also the higher glycogen content may account for the increased muscle weight of the Compacts; therefore, in this present study we aimed to analyze the muscle cellularity of the Compact mice focusing on the glycogen content and the fiber-type specific glycogen distribution. Here we show that the glycogen content of the IIB fibers was the highest among the fast fibers in the tibialis anterior (TA) muscle. The myostatin deficiency of Compact mice resulted in elevated glycogen content of the TA muscle but the average glycogen content of the individual fibers remained unchanged despite the marked glycolytic shift.

\section{Materials and Methods}

\section{Animals and sample collection}

This investigation conformed to the National Institutes of Health Guide for the Care and Use of Laboratory Animals (NIH Pub. No. 85-23, revised 1996) and was approved by the local ethics committee at the University of Szeged. We performed our experiments on 12 week-old male myostatin mutant Compact $(47.3 \pm 0.76 \mathrm{~g}$; $\mathrm{n}=8)$ and wild type BALB/c $(25.0 \pm 0.58 \mathrm{~g} ; \mathrm{n}=4)$ mice. The origin and the selection procedure of the Compact mice were described by Baán and colleagues. ${ }^{16}$ Briefly, the Compact line was selected initially in Berlin on the basis of high protein mass and hypermuscularity. The Hungarian subpopulation of this Compact line was inbred and kept for more than 20 years in the Institute for Animal Biology, Agricultural Animal Center (Gödöllő, Hungary), while they have been breeding since 2010 in the
Correspondence: Anikó Keller-Pintér, University of Szeged, Faculty of General Medicine, Department of Biochemistry, Dóm tér 9., H-6720 Szeged, Hungary.

Tel. 36.62.545097 - Fax: +36.62.545097.

E-mail: keller.aniko@med.u-szeged.hu

Key words: Compact mice, fiber-type, GDF-8, glycogen, muscle, myostatin.

Contributions: AKP, LM, GM, LD, study concept and design; TK, JB, data collection; TK, analysis and interpretation of data; TK, AKP, LM, manuscript drafting; AKP, LM, LD, critical revision of the manuscript; AKP, study supervision.

Acknowledgments: the authors would thank Zita Makráné Felhő and Istvánné Balásházi for their excellent technical assistance and Ernő Zádor and Mónika Kiricsi for the anti-MyHC antibodies.

Funding: this research was supported by the European Union and the State of Hungary, cofinanced by the European Social Fund in the framework of TÁMOP 4.2.4. A/2-11-1-2012-0001 National Excellence Program (to AKP), TÁMOP4.2.2.A-11/1/KONV-2012-0035 and Richter Gedeon Centenary Foundation.

Received for publication: 11 December 2013. Accepted for publication: 18 April 2014.

This work is licensed under a Creative Commons Attribution NonCommercial 3.0 License (CC BYNC 3.0).

(C) Copyright T. Kocsis et al., 2014

Licensee PAGEPress, Italy

European Journal of Histochemistry 2014; 58:2353 doi:10.4081/ejh.2014.2353

Department of Biochemistry, Faculty of General Medicine, University of Szeged (Szeged, Hungary). The non-selected BALB/c mice exhibiting wild-type myostatin gene were obtained from the Biological Research Centre of the Hungarian Academy of Sciences (Szeged, Hungary). The animals were kept under controlled temperature with $12 / 12 \mathrm{~h}$ light/dark cycles, and were fed standard chow and tap water ad libitum. Mice were anaesthetized by intraperitoneal injection of chloral hydrate $(3 \%$ chloral hydrate, $0.15 \mathrm{~mL} / 10 \mathrm{~g}$ body weight). The quadriceps femoris (QF), biceps femoris (BF), gastrocnemius (Gastro), tibialis anterior (TA) and extensor digitorum longus (EDL) muscles were removed and frozen immediately in isopentane cooled by liquid nitrogen and stored at $-80^{\circ} \mathrm{C}$ until further processing. The TA muscle from the right hindlimb was used for the spectrophotometric determination of glycogen and protein content, whereas morphological analysis [Periodic Acid Schiff (PAS)-staining, immunohistochemistry] was performed on the contralateral TA muscle. 


\section{Glycogen and protein determina- tion by spectrophotometry}

Muscle glycogen was measured as glucose residues after acidic hydrolysis by a standard enzymatic assay. Briefly, following cryogenic milling the samples were digested for $1.5 \mathrm{~h}$ in $2.0 \mathrm{M} \mathrm{HCl}(250 \mu \mathrm{L} \mathrm{HCl} / 10 \mathrm{mg}$ muscle tissue $)$ at $100^{\circ} \mathrm{C}$. After lysis the samples were cooled to room temperature and neutralized by adding of equal amount of $2.0 \mathrm{M} \mathrm{NaOH}$. Thereafter, the samples were centrifuged for $10 \mathrm{~min}$ at 21,000× g (Hettich Universal 320R, DJB, Labcare Ltd., Buckinghamshire, UK) at room temperature, and the supernatants were removed. The concentration of glucose was determined from the supernatant by Hexokinase kit (Roche, Mannheim, Germany).

Muscles were homogenized in a buffer $(0.1$ M Tris-HCl pH 8.0, 0.01 M EDTA, 10\% SDS) containing protease inhibitor cocktail (SigmaAldrich, St. Louis, MO, USA) to extract the total protein amount. The incubation of the samples at $99^{\circ} \mathrm{C}$ for $60 \mathrm{~s}$ was followed by centrifugation at $11,000 \times \mathrm{g}$ for $5 \mathrm{~min}$ (Hettich Universal 320R, DJB, Labcare Ltd.) at room temperature to remove cellular debris. The supernatant protein content was determined by BCA Protein Assay Reagent (Thermo Scientific, Rockford, IL, USA) in agreement with the manufacturer's instructions.

Spectrophotometry was performed on Fluostar Optima (BMG Labtech, Ortenberg, Germany) and data were analyzed on Mars Data Analysis Software (BMG Labtech).

\section{PAS-staining}

Glycogen was detected by performing PASstaining on $10 \mu \mathrm{m}$ cryosections of the TA muscle. Sections were fixed for $1 \mathrm{~h}$ at $4^{\circ} \mathrm{C}$ in $3.7 \%$ formaldehyde in ethanol immediately to avoid glycogen breakdown, and incubated for $15 \mathrm{~min}$ with $0.5 \%$ periodic acid (Sigma Aldrich) at room temperature, followed by $5 \mathrm{~s}$ washing in tap water and deionized water four times. Then the sections were incubated in Schiff's reagent for $1 \mathrm{~h}$ at room temperature, followed by $5 \mathrm{~min}$ incubation (twice) in potassium metabisulfite in deionized water. Thereafter, gently washing of the slides for $10 \mathrm{~s}$ under running hand-warm tap water was followed by washing three times for $5 \mathrm{~s}$ in deionized water. Finally, the sections were incubated for $30 \mathrm{~s}$ in $50,70,90,100 \%$ ethanol and for $15 \mathrm{~s}$ in toluol, then were mounted with Entellan.

\section{Immunohistochemistry}

Fiber-type analysis was performed on $10 \mu \mathrm{m}$ serial cryosections of the midbelly region of TA muscle. The sections were blocked in 5\% nonfat milk powder (BioRad, Berkeley, CA, USA) in PBS, and then incubated with mouse monoclonal primary antibodies. BA-D5 (1:25), sc-71 (1:25) and BFF3 (1:5) primary antibodies were used, specific for Myosin Heavy Chain I (MyHCI, slow oxidative), MyHCIIA (fast oxidative) and MyHCIIB (fast glycolytic). ${ }^{17,18}$ After incubation with the peroxidase-conjugated secondary antibody (rabbit anti-mouse; Dako, Glostrup, Denmark), the immunocomplexes were visualized by 3,3 '-diaminobenzidine. We could not detect BA-D5 positive (MyHCI) fibers in the TA muscle; therefore, the fibers stained with neither MyHCIIA nor MyHCIIB were considered as MyHCIIX fibers.

\section{Determination of the glycogen con- tent of the individual muscle fibers}

Photos were taken with $10 \mathrm{x}$ objective using a Nikon Labophot-2 microscope equipped with Olympus DP71 camera. The full cross sectional areas (CSAs) of the muscles were reconstructed from the microscopic images by Cell*B software (Olympus DP Soft software, ver. 3.2, Soft Imaging System $\mathrm{GmbH}$, Munster, Germany).

The glycogen content of an individual fiber $\left(0 D^{*} \mu \mathrm{m}^{2}\right)$ can be predicted by multiplying the average intensity of PAS-staining by the CSA of the fiber. Average intensity (optical density, OD) of PAS-staining (0-1 OD), CSAs of all fibers $\left(\mu \mathrm{m}^{2}\right)$ and fiber numbers were determined on the whole muscle CSA on greyscale converted PAS-stained, panoramic images by Digimizer software (Medcalc software, Mariakelke, Belgium).

The fiber-type specific average intensity of PAS-staining and the CSAs of the different fiber types were determined on 2-3 representative microscopic fields of both superficial and deep regions of the TA muscle (400-1000 fibers/sample, $14-17 \%$ of total fiber number with $10 \mathrm{x}$ objective). These muscle regions were selected for analysis because the tibialis anterior muscle shows an increasing gradient of fibres having a high oxidative enzyme activity proceeding from the superficial to the deep region of the muscle. ${ }^{19}$ Both the sc-71 (MyHCIIA) and the BF-F3 (MyHCIIB) stained regions were matched with the PAS-stained identical regions on serial sections to analyze the glycogen content of the different fiber types. The glycogen-index (\%) of a fiber-type was defined as the average $0 D^{*} \mu m^{2}$ value of a fiber type ${ }^{*}$ fiber type proportion / summarized $\mathrm{OD}^{*} \mathrm{\mu m}^{2 *}$ fiber type proportion values to compare the total glycogen content of the different fiber types.

\section{Statistical analysis}

Statistical evaluations were performed by unpaired $t$-test to assess the effect of Compact mutation on body weight, absolute and normalized weights of muscles, total protein and glycogen content of the whole TA muscle determined by spectrophotometry, relative distribution of the different fiber types, average inten- sity of PAS-staining of the fibers on whole muscle CSA, average area of the fibers, average glycogen content of the fibers and total glycogen content of the TA muscle based on PAS-staining. The applied statistical method was one-way ANOVA followed by NewmanKeuls post hoc test (GraphPad Software, Inc., San Diego, CA, USA) for the analysis of the fiber-type specific alterations of average area of fibers, average intensity of PAS-staining and average glycogen content of the different fiber types on the whole CSA or in the superficial and deep regions of TA muscle; and for the analysis of glycogen index. All data is expressed as means $\pm \mathrm{SEM}$.

\section{Results}

\section{Body weight and muscle weights}

First, we compared the body weights and the weights of the investigated hindlimb muscles of the Compact mice and the wild type BALB/c line. Here we show that the body weight of the Compact mice is almost two-fold larger $(\mathrm{P}<0.001$, Table 1). The absolute weights of the investigated hindlimb muscles such as quadriceps femoris, biceps femoris, gastrocnemius, tibialis anterior and extensor digitorum longus were at least two-fold larger in Compacts $(\mathrm{P}<0.001 ;$ Table 1$)$. The muscle weights normalized to body weight significantly increased in Compact QF (1.23-fold; $\mathrm{P}<0.001)$, BF (1.3-fold; $\mathrm{P}<0.001)$, Gastro (1.34fold; $\mathrm{P}<0.001)$, TA $(1.35$-fold; $\mathrm{P}<0.001)$ and EDL (1.3-fold; $\mathrm{P}<0.01)$ muscles compared to $\mathrm{BALB} / \mathrm{c}$ line (Table 1).

\section{Muscle characteristics in Compact mice}

The Compact line was selected for high protein content and hypermuscularity. ${ }^{11}$ Beside the increased protein amount the higher glycogen content may account for the increased muscle weight of the Compact mice. Thus, we measured both total protein and glycogen amounts of the fast glycolytic TA muscle by spectrophotometry. Similarly to the two-fold increase in total protein content $(\mathrm{P}<0.001$; Table 1), the amount of glycogen was 1.9 -fold greater $(\mathrm{P}<0.01$; Table 1$)$ in the Compact $\mathrm{TA}$ muscle compared to the wild type. The remarkable increase in protein content was even higher than that of glycogen level proving the significance of protein accretion in the phenotype of Compact mice. Next we tested, whether the higher glycogen amount of the Compact muscle is caused by the changes in the glycogen content of the individual muscle fibers. To analyze this, first we determined the OD of PAS-staining and fiber sizes on the whole CSA of the TA muscle (Figure 1). The average 
intensity of PAS-staining did not show significant change in Compact muscle fibers compared to those of the wild type muscle (Figure 1C), and no significant difference was observed regarding the average CSAs of the fibers either (Figure 1D).

The average intensity of PAS-staining multiplied by the value of fiber size is suitable to estimate the average glycogen content of the individual fibers. Comparing the fibers average glycogen content we could not detect any significant changes between the two groups of animals (Figure 1E). Similar to previously reported results, ${ }^{15,16}$ the fiber number increased 1.7-fold in Compact animals $(\mathrm{P}<0.001$; Table 2). Multiplying the average glycogen content of the individual fibers by the increased fiber number we detected a similar, two-fold increase in the glycogen content of the whole muscle by PAS-staining $(\mathrm{P}<0.05$; Figure $1 F$ ) as measured by spectrophotometry (Table 1).

Taken together, the average glycogen content of the individual fibers did not change; therefore, the increased fiber number can explain the elevated glycogen amount of the Compact TA muscle.

\section{Fiber-type specific alterations in muscle cellularity}

To analyze the possible presence of the fiber-type specific alterations of the glycogen content, first we examined the fiber type composition of TA muscle and measured the CSAs of the different fiber types on serial sections immunostained by antibodies against the different isoforms of MyHCs (Figure 2). Regarding the fiber composition of the Compact muscle, we could not detect the slow MyHCI isoform in TA muscles (Table 2) in accordance with the literature. ${ }^{20}$ The ratio of IIB fibers $(\mathrm{P}<0.01$; Table 2$)$ increased 1.6-fold with concomitant decreases of IIX $(\mathrm{P}<0.05$; Table 2) and IIA fibers $(\mathrm{P}<0.001$; Table 2$)$ indicating a glycolytic shift. Because the number of IIA fibers in Compact TA was very low (Table 2), we could not perform statistical analysis on this type of fibers. Remarkable differences were observed comparing the sizes of the different fiber types on the whole CSA of the muscle. As in wild type animals, the CSA of type IIB fibers was 1.44-fold larger than those of type IIX; and IIX fibers were 1.52-fold larger than the IIA ones $(\mathrm{P}<0.01$; Figure $3 \mathrm{~A})$. In Compact mice we found a similar result: the CSA of type IIB fibers was 1.62-fold bigger than those of IIX fibers $(\mathrm{P}<0.001$; Figure 3A). Comparing the two mice lines we did not find any differences between the average size of either IIB or IIX fibers. The frequency distributions of both the IIX and IIB fiber sizes showed similar shapes in Compact and wild type mice (Figure $3 \mathrm{~B}, \mathrm{C}$ ). The histograms of IIX fibers (Figure $3 \mathrm{~B}$ ) revealed right-skewed distribution in both groups of animals. In case of IIB fibers the bellshaped curves were wider in Compact and BALB/c animals (Figure 3C) than those of IIX

fibers (Figure 3B). The superficial and deep regions of the TA muscle consist of different fiber types; ${ }^{10,19}$ therefore, we performed our measurements on both regions as well.

Table 1. Body weight, absolute and normalized weights of the quadriceps femoris, biceps femoris, gastrocnemius, tibialis anterior and extensor digitorum longus muscles of 12 week-old, male BALB/c and Compact mice $(n=4-8)$. Total protein amount and glycogen content of the TA muscle determined by spectrophotometry in BALB/c and Compact mice $(n=4-6)$.

\begin{tabular}{lccc}
\hline Item & BALB/c & Compact & P value \\
Bw (g) & $25.0 \pm 0.58$ & $47.3 \pm 0.76$ & $<0.001$ \\
Muscle weights & & & \\
QF (mg) & $206 \pm 6.33$ & $470 \pm 12.19$ & $<0.001$ \\
BF (mg) & $167 \pm 7.23$ & $420 \pm 13.83$ & $<0.001$ \\
Gastro (mg) & $140 \pm 3.82$ & $352 \pm 7.42$ & $<0.001$ \\
TA (mg) & $45.3 \pm 0.73$ & $114 \pm 1.48$ & $<0.001$ \\
EDL (mg) & $10.0 \pm 0.65$ & $23.5 \pm 0.35$ & $<0.001$ \\
\hline Muscle weights/bw & & & \\
QF/bw (mg/g) & $8.0 \pm 0.12$ & $10.0 \pm 0.20$ & $<0.001$ \\
BF/bw (mg/g) & $6.5 \pm 0.12$ & $8.5 \pm 0.37$ & $<0.001$ \\
Gastro/bw (mg/g) & $5.5 \pm 0.11$ & $7.4 \pm 0.12$ & $<0.001$ \\
TA/bw (mg/g) & $1.77 \pm 0.03$ & $2.40 \pm 0.04$ & $<0.01$ \\
EDL/bw (mg/g) & $0.39 \pm 0.02$ & $0.51 \pm 0.01$ & $<0.001$ \\
Total protein amount in TA (mg) & $9.2 \pm 0.42$ & $18.9 \pm 0.38$ & $<0.01$ \\
\hline Total glycogen content in TA (mg) & $0.23 \pm 0.04$ & $0.44 \pm 0.02$ & \\
\hline
\end{tabular}

Bw, body weight; QF, M. quadriceps femoris; BF, M. biceps femoris; Gastro, M. gastrocnemius; TA, M. tibialis anterior; EDL, M. extensor digitorum longus. Values are mean \pm SEM.

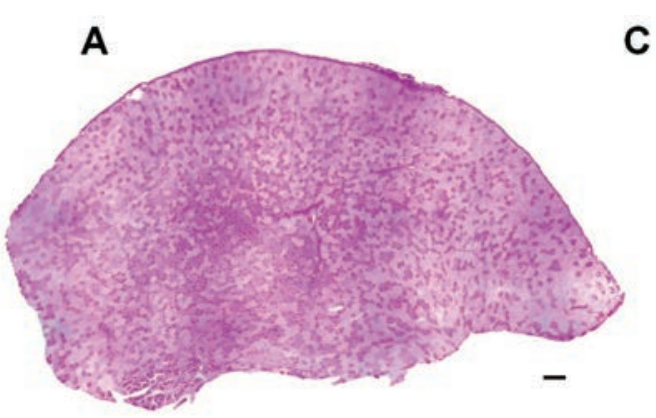

C

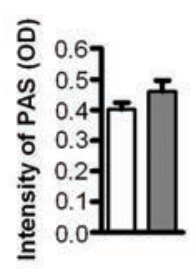

B

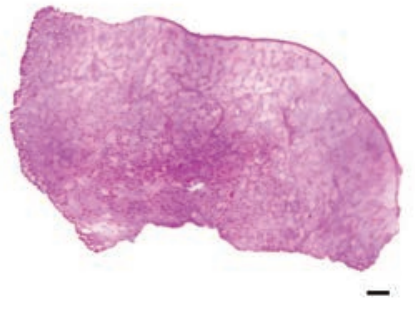

D

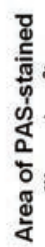

E

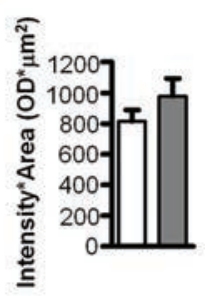

Figure 1. Representative panoramic images of Compact (A) and BALB/c (B) tibialis anterior muscles stained by PAS. Average intensity of PAS-staining of the fibers on whole muscle cross sectional area (C), average area of the fibers (D), average glycogen content of the individual fibers $(\mathrm{E})$ and total glycogen content of the tibialis anterior muscle $(\mathrm{F})$ based on PAS-staining $(n=3-3)$. Data are reported as mean $\pm S E M ;{ }^{* *} P<0.01 ;{ }^{* * *} P<0.001$. Scale bars: $200 \mu \mathrm{m}$. 
According to fiber size we found similar results, i.e., the area of IIB fibers was the greatest (IIA $v s$ IIX, IIX $v s$ IIB, IIA $v s$ IIB in $\mathrm{BALB} / \mathrm{c}$ : superficial $\mathrm{P}<0.01$, deep: $\mathrm{P}<0.05$; IIX vs IIB in Compact: superficial $\mathrm{P}<0.001$; deep $\mathrm{P}<0.01$; Figure $4 \mathrm{~A}$ ), with no differences between the superficial and deep regions of the TA muscle in either mouse line, and no changes were observed regarding the fiber sizes of the two mice strains (Figure 4A).

To compare the effect of the Compact mutation on the fiber-type specific glycogen content of the muscle, we measured the average intensity of PAS-staining of the different fiber types on serial cryosections stained with antibodies against the different types of MyHC isoforms and PAS, respectively (Figure 2). The average intensity of PAS-staining showed a similar decreasing tendency in a rank order IIA $>$ IIX $>$ IIB on the whole CSA of the muscle: IIB fibers exhibited the weakest OD (Figure $3 \mathrm{~B})$, as in the superficial and deep regions (Figure 4B) in both mouse lines. The average intensity of PAS-staining of the different fiber types did not show significant differences between the Compact and BALB/c fibers on the whole muscle CSA (Figure 3D), and no significant changes could be observed comparing the PAS intensity of the fiber types in the superficial and deep regions either (Figure 4B). However, the frequency distribution revealed differences despite the similar average values of intensity of PAS-staining in Compact and wild type mice: the Compact TA muscle contained populations of IIX fibers with higher, and IIB fibers with both higher and lower intensity of PAS-staining resulting in a wider distribution in mutant animals (Figure 3 E,F). Next, the average glycogen content of the different fiber types was compared in both strains. The average glycogen content of the IIB fibers was greater than those of IIX fibers, whereas the IIA was the lowest in the superficial and deep regions of wild type muscle (Figure 4C). The results indicated significant difference in the superficial region of $B A L B / c$ animals (IIA $v s$ IIX, IIX $v s$ IIB, IIA $v s$ IIB; $\mathrm{P}<0.05$; Figure $4 \mathrm{C}$ ). The fiber type specific glycogen content on the whole CSA revealed similar tendency; however, without any significant changes (Figure 3G). In parallel with the frequency distribution of PAS intensity (Figure $3 \mathrm{E}, \mathrm{F})$, histograms of the average glycogen content (Figure $3 \mathrm{H}, \mathrm{I}$ ) showed that the Compact TA muscle contains a population of IIX fibers with higher, and IIB fibers with both higher and lower glycogen content .

Finally, we compared the total glycogen content of the different fiber types in the TA muscle. The glycogen index of IIB fibers was the highest, so that the IIB fibers stored the most glycogen in both BALB/c and Compact mice. The glycogen index value of IIX fibers was 2.7- fold larger $(\mathrm{P}<0.01)$ in $\mathrm{BALB} / \mathrm{c}$ mice compared to Compact animals (Figure 5) in line with the higher number of IIX fibers present in BALB/c mice. In contrast the glycogen index of IIB fibers was 1.5-fold bigger in Compacts $(\mathrm{P}<0.01)$ in good accordance with the increased proportion of IIB fibers in Compact mice.

\section{Discussion}

The Compact mutant mice carry a naturally occurring 12-bp non-frame shift deletion in the prodomain region of the precursor myostatin resulting hypermuscularity. ${ }^{12}$ Additional modifiers seem to be present to determine the full expression of the phenotype; however, these modifier genes have not been identified yet. ${ }^{13,14}$

The condition of the double muscled phenotype has been observed in several species. Natural mutations of myostatin can effect of the bioactive domain of myostatin: the mutation in Belgian Blue [nt821(del11)] causes a frame-shift virtually eliminating all of the mature, active regions of the molecule, whereas Piedmontese cattle presents a G938A transition resulting in a full-length, however misfolded and dysfunctional myostatin. ${ }^{4,21}$ Mutations in the pro-peptide leading an early

Table 2. Relative distribution of the different fiber types in BALB/c and Compact mice.

$\begin{array}{lccc}\text { Item } & \text { BALB } / \mathrm{c} & \text { Compact } & \text { P value } \\ \text { Total fiber number } & 3100 \pm 52.48 & 5200 \pm 227.6 & <0.001 \\ \text { Fiber type frequency (\%) } & & 0 & \\ \text { MyHCI } & 0 & 1 \pm 0.05 & <0.001 \\ \text { MyHCIIA } & 3 \pm 0.09 & 19 \pm 3.25 & <0.05 \\ \text { MyHCIIX } & 47 \pm 2.59 & 80 \pm 3.29 & <0.01 \\ \text { MyHCIIB } & 50 \pm 2.55 & \end{array}$

MyHC, Myosin Heavy Chain. Values are mean \pm SEM; $n=3-3$.
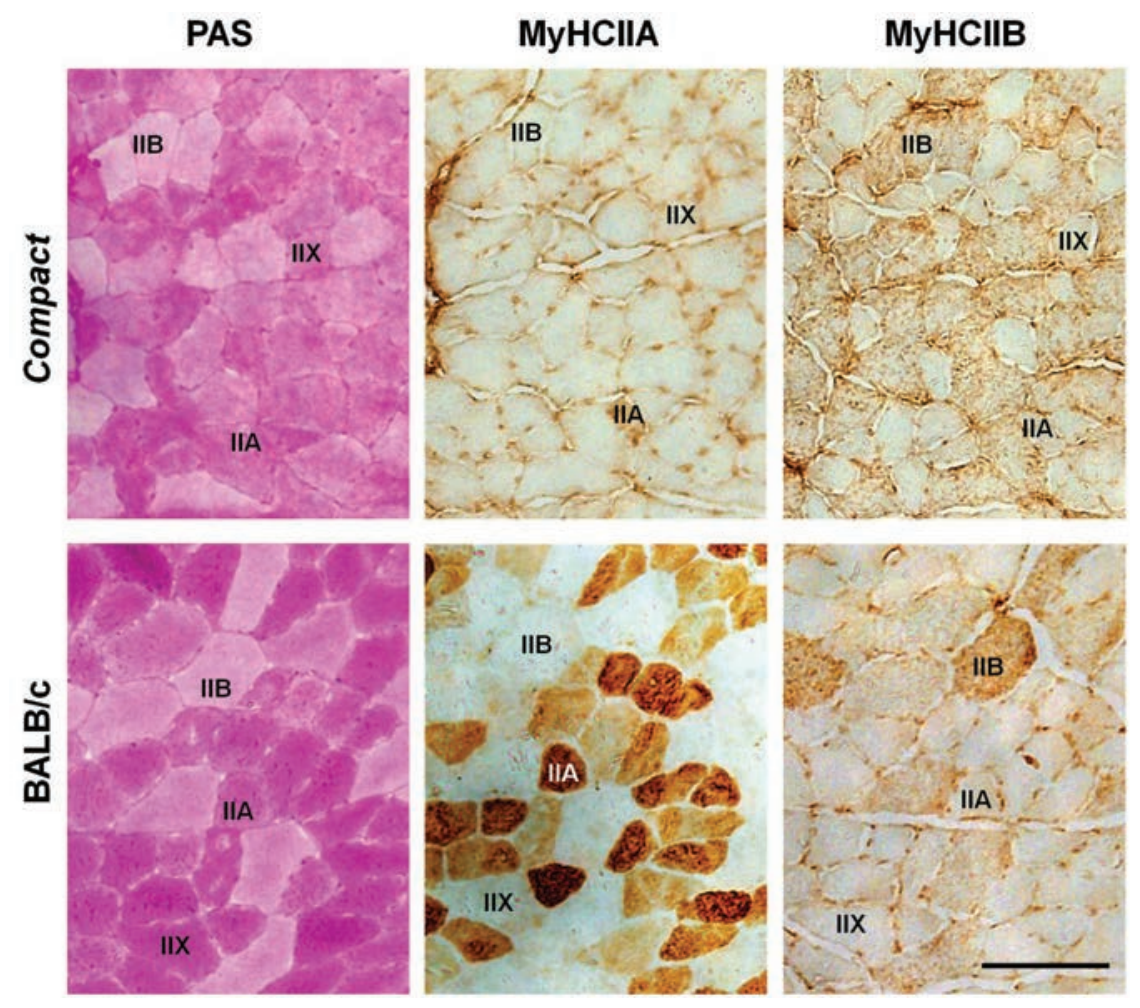

Figure 2. Representative microscopic images of the deep region of tibialis anterior muscle of BALB/c and Compact mice. Serial sections were stained by either PAS or antibodies against MyHCIIA and MyHCIIB isoforms. Representative fibers are marked as IIA, IIB and IIX. Scale bar: $100 \mu \mathrm{m}$. 
STOP codon [nt419(del7-ins10), Q204X, and E226X] have also been identified. ${ }^{22}$ The biologically active growth factor domain of myostatin is unaffected by Compact mutation, therefore the loss of myostatin activity cannot be explained by disruption of the growth factor bioactive domain; however, the Compact mutation can lead to misfolding, or defect in secretion and mistargeting of mature myostatin. ${ }^{2}$

In the present study we depict, similarly to previous observations, ${ }^{15,16,23,24}$ that the body and muscle weights of Compact animals increased compared to a mice line exhibiting wild type myostatin gene. Thus, the disproportionate increase of muscle weight can lead to increased body weight of Compact mice. Originally the Compact line was selected for hypermuscularity and high protein content at the Technical University in Berlin. ${ }^{11}$ Rehfeldt and colleagues introgressed the Compact mutation into the DUHi mice line. ${ }^{15}$ The hypermuscularity of this Compact-DUHi mice was characterized by muscle fiber hyperplasia rather than hypertrophy in fast glycolytic longissimus dorsi and rectus femoris muscles, and by balanced increases in myonuclear proliferation and protein accretion. ${ }^{15}$ However, no reports have so far examined the glycogen amount of the Compact muscles and the glycogen content of the individual fiber types. Here we show that the total glycogen amount of the TA muscle is markedly greater in Compact than in wild type mice. The phenotype selection on extreme muscle mass in DU6P mice

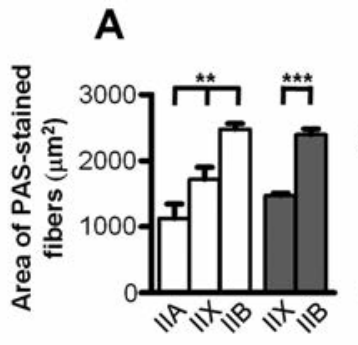

B

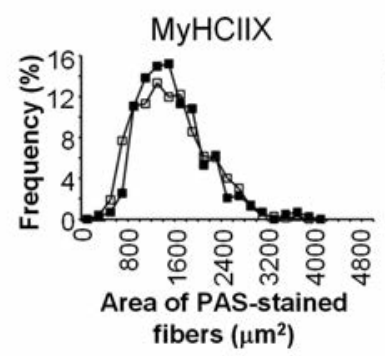

E
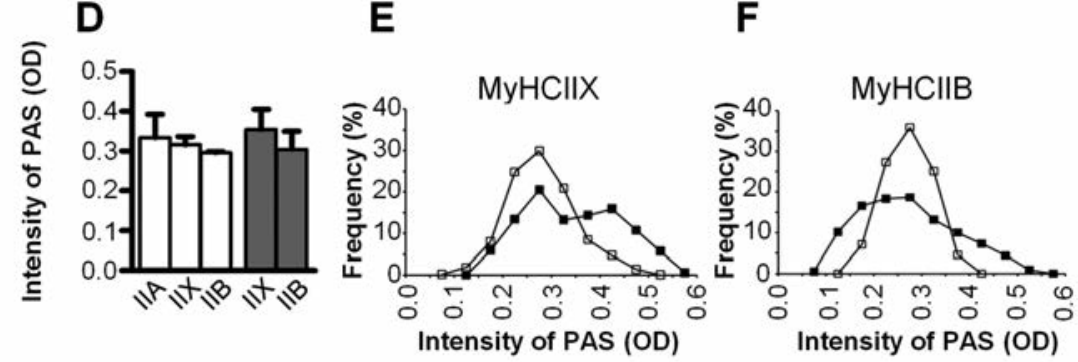

G

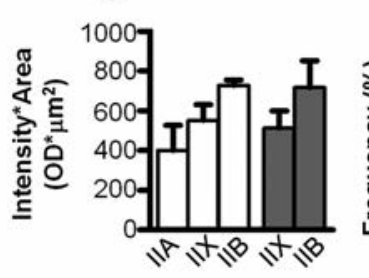

H

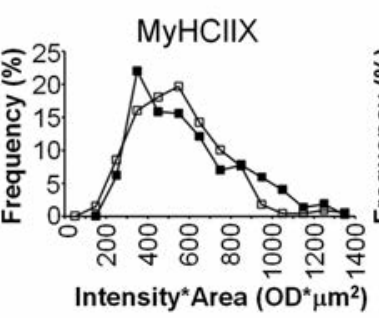

I

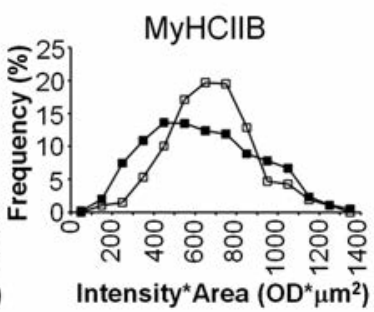

BALB/c

Compact

๑ BALB/c

Compact

Figure 3. Average area of fibers (A), average intensity of PAS-staining (D) and average glycogen content of the different fiber types $(G)$ on the whole cross sectional area of the tibialis anterior muscle in the Compact and BALB/c mice $(n=3-3)$. Histograms $(\mathrm{B}, \mathrm{C}, \mathrm{E}, \mathrm{F}, \mathrm{H}, \mathrm{I})$ show the frequency distribution of the defined parameters. Data are reported as mean $\pm \mathrm{SEM}$; ${ }^{* *} \mathbf{P}<0.01 ;{ }^{* * *} \mathbf{P}<0.001$. revealed the co-evolution of high glycogen and protein content of the muscle. ${ }^{25}$ This finding is in line with our observation on the simultaneous increase of protein and glycogen contents in Compact muscle. The PI3K/Akt signaling plays a central role in integrating anabolic and catabolic responses in skeletal muscle influencing muscle mass. ${ }^{26,27}$ PTEN (Phosphatase and tensin homolog) negatively affects the

A

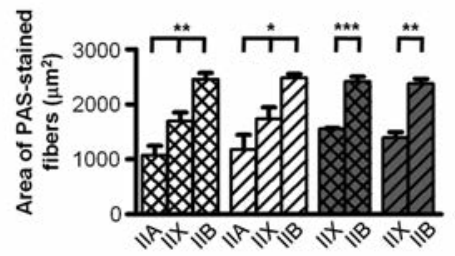

B

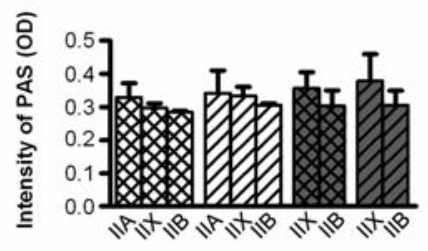

C

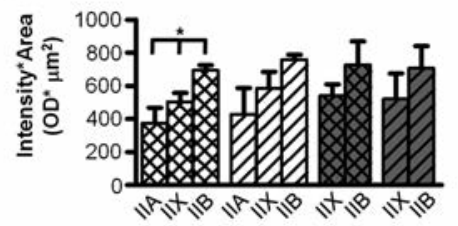

$\begin{array}{ll}\text { ख BALB/c superficial } & \mathbb{Z} \text { BALB/c deep } \\ \text { CD Compact superficial } & \text { Compact deep }\end{array}$

Figure 4. Average area of fibers (A), average intensity of PAS-staining (B) and average glycogen content of the fiber types $(C)$ in the superficial and deep regions of the tibialis anterior muscle in Compact and BALB/c mice $(n=3-3)$. Data are reported as mean \pm SEM; ${ }^{*} \mathbf{P}<0.05 ;{ }^{* *} \mathbf{P}<0.01$; ${ }^{* * *} \mathrm{P}<\mathbf{0 . 0 0 1}$.

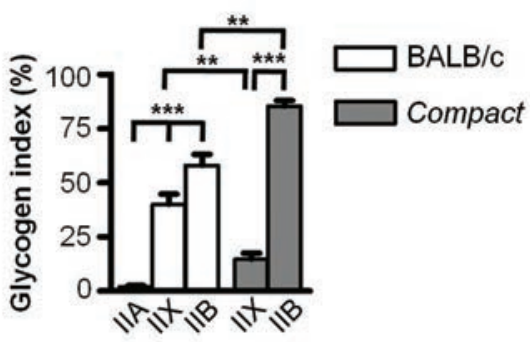

Figure 5. Glycogen index of the different (IIA, IIX and IIB) fiber types of the tibialis anterior muscle in $\mathrm{BALB} / \mathrm{c}$ and Compact mice. Data are reported as mean \pm SEM $\left(\mathbf{n}=3-3,{ }^{* *} \mathbf{P}<0.01 ;{ }^{* * *} \mathbf{P}<0.001\right)$. 
PI3K/Akt pathway, ${ }^{28}$ moreover, Sawitzky and colleagues $^{25}$ identified PTEN as a gate keeper molecule in the co-evolutionary regulation of high glycogen and protein content.

In order to analyze the glycogen content of the individual muscle fibers the intensity of PAS-staining was measured. However, the value of the average OD of the pixels within a fiber cannot reflect the glycogen content of the whole muscle fiber; the OD value should be multiplied by the fiber size. In accordance with the literature ${ }^{15}$ we did not find differences comparing the fiber sizes of the Compact and wild type TA muscles. Furthermore, based on our results, the average glycogen content of the muscle fibers remained unchanged in Compacts compared to wild type mice; therefore, the increased number of fibers can explain the higher total glycogen amount of the Compact TA muscle. In addition to controlling muscle mass, myostatin also appears to regulate muscle fiber type composition postnatally by regulating the expression of both MyoD and MEF2C genes. ${ }^{29}$ Histological analysis revealed increased proportion of type IIB fibers in tibialis anterior, biceps femoris ${ }^{29}$ and EDL muscles $^{23}$ of myostatin knockout mice, or in double-muscled cattle. ${ }^{9,10}$ In accordance with our observations, earlier studies described that the Compact mutation induces a glycolytic shift in the phenotype of skeletal muscle, ${ }^{15,16}$ similarly to the situations evoked by the lack or the dysfunction of myostatin. Rehfeldt and her group reported that the number of white and intermediate fibers increased in rectus femoris and longissimus dorsi muscles of 70-day-old Compact mice, whereas the density of the blood capillaries decreased..$^{15}$ Despite the fact that the Compact mice are hypermuscular and their muscles consist of significantly more fibers, the specific force generation decreased in Compact animals. ${ }^{23}$ According to our results, the proportion of fast glycolytic fibers in TA muscle of Compact mice increased; however, their individual glycogen content did not.

Regarding the size of the different fiber types we found similar results in both Compact and $\mathrm{BALB} / \mathrm{c}$ mice: it increased in the rank order IIA $<$ IIX $<$ IIB among the fast fibers. In accordance with our observations, the area of IIB fibers was the biggest in the rectus femoris muscle of Compact-DUHi mice. ${ }^{15}$ The average glycogen content of the individual muscle fibers showed similar tendency as the fiber size (i.e., increased in the rank order IIA $<$ IIX $<$ IIB). Thus, the IIB fibers contained the most glycogen in both Compact and BALB/c mice, correlating with the biochemical properties of the fibers. Comparing the superficial and deep regions of the TA muscle we found similar results according to the fiber size, intensity of PAS-staining, as well as the glycogen content of the fibers. It is important to note that the measurement of the PAS intensity alone cannot predict clearly the glycogen content of the fibers (i.e., type IIB fibers have lower average PAS intensity but higher fiber size leading to larger glycogen content than that of IIX fibers). Only a few data are available on the glycogen content of the different types of fast fibers, mainly reported in human muscle biopsies. In accordance with our results, type IIA fibers showed more intense PAS-staining than IIB fibers in human biopsy samples. ${ }^{30}$

In conclusion, the phenotype of the Compact muscle is shifted towards glycolysis, similarly to previous observations. ${ }^{15,16}$ Among the fibers of the TA muscle, IIB fibers contained the most glycogen, accompanied by their increased number in Compact muscle. The myostatin mutation was associated with elevated glycogen content in the TA muscle without any change in the glycogen content of individual fibers despite the marked glycolytic shift.

\section{References}

1. McPherron AC, Lawler AM, Lee SJ. Regulation of skeletal muscle mass in mice by a new TGF- $\beta$ superfamily member. Nature 1997;387:83-90.

2. Schuelke M, Wagner KR, Stolz LE, Hübner C, Riebel T, Kömen W, et al. Myostatin mutation associated with gross muscle hypertrophy in a child. N Engl $\mathrm{J}$ Med 2004;350:2682-8.

3. McPherron AC, Lee SJ. Double muscling in cattle due to mutations in the myostatin gene. Proc Natl Acad Sci USA 1997;94: 12457-61.

4. Kambadur R, Sharma M, Smith TP, Bass JJ. Mutations in myostatin (GDF8) in double-muscled Belgian Blue and Piedmontese cattle. Genome Res 1997;7: 910-6.

5. Shelton GD, Engvall E. Gross muscle hypertrophy in whippet dogs is cause by a mutation in the myostatin gene. Neuromuscul Disorders 2007;17:721-2.

6. Stinckens A, Luyten T, Bijttebier J, Van den Maagdenberg K, Dieltiens D, Janssens S, et al. N. Characterization of the complete porcine MSTN gene and expression levels in pig breeds differing in muscularity. Anim Genet 2008;39:586-96.

7. Shahin KA, Berg RT. Growth patterns of muscle, fat and bone and carcass composition of double muscled and normal cattle. Can J Anim Sci 1985;65:279-93.

8. Wegner J, Albrecht E, Fiedler I, Teuscher F, Papstein HJ, Ender K. Growth and breedrelated changes of muscle-fiber characteristics in cattle. J Anim Sci 2000;78:1485-96.

9. Holmes JH, Ashmore CR. A histochemical study of development of muscle fibre type and size in normal and double muscled cattle. Growth 1972;36:351-72.

10. Matsakas A, Mouisel E, Amthor H, Patel K. Myostatin knockout mice increase oxidative muscle phenotype as an adaptive response to exercise. J Muscle Res Cell Motil 2010;31:111-25.

11. Bünger L, Laidlaw A, Bulfield G, Eisen EJ, Medrano JF, Bradford GE, et al. Inbred lines of mice derived from long-term on growth selected lines unique resources for mapping growth genes. Mamm Genome 2001;12:678-86.

12. Szabó G, Dallmann G, Müller G, Patthy L, Soller M, Varga L. A deletion in the myostatin gene causes the compact (Cmpt) hypermuscular mutation in mice. Mamm Genome 1998;9:671-2.

13. Varga L, Müller G, Szabó G, Pinke 0, Korom E, Kovács B, et al. Mapping modifers affecting muscularity of the myostatin mutant (MstnCmpt-dl1Abc) compact mouse. Genetics 2003;165:257-67.

14. Varga L, Pinke 0, Müller G, Kovács B, Korom E, Szabó G, et al. Mapping a syntenic modifier on mouse chromosome 1 influencing the expressivity of the compact phenotype in the myostatin mutant (MstnCmpt-dl1Abc) Compact mouse. Genetics 2005;169:489-93.

15. Rehfeldt C, Ott G, Gerrard DE, Varga L, Schlote W, Williams JL, et al. Effects of the Compact mutant myostatin allele Mstn(Cmpt-dl1Abc) introgressed into a high growth mouse line on skeletal muscle cellularity. J Muscle Res Cell Motil 2005;26:103-112.

16. Baán JA, Kocsis T, Keller-Pintér A, Müller G, Zádor E, Dux L, et al. The Compact mutation of myostatin causes a glycolytic shift in the phenotype of fast skeletal muscles. J Histochem Cytochem 2013;1:889-900.

17. Smerdu V, Soukup T. Demonstration of myosin heavy chain isoforms in rat and humans: the specificity of seven available monoclonal antibodies used in immunohistochemical and immunoblotting methods. Eur J Histochem 2008:52:179-90.

18. Schiaffino S, Gorza L, Sartore S, Saggin L, Ausoni S, Vianello M, et al. Three myosin heavy chain isoforms in type 2 skeletal muscle fibers. J Muscle Res Cell Motil 1989:10:197-205.

19. Ishihara A, Hirofuji C, Nakatani T, Itoh K, Itoh M, Katsuta S. Effects of running exercise with increasing loads on TA muscle fibres in mice. Exp Physiol 2002;87:113-6.

20. Carlson CJ, Booth FW, Gordon SE. Skeletal muscle myostatin mRNA expression is fiber-type specific and increases during hindlimb unloading. Am J Physiol Regul Integr Comp Physiol 1999;277:R601-6.

21. Berry C, Thomas M, Langley B, Sharma M, 
Kambadur R. Single cysteine to tyrosine transition inactivates the growth inhibitory function of Piedmontese myostatin. Am J Physiol Cell Physiol 2002;283:C135-41.

22. Grobet L, Poncelet D, Royo LJ, Brouwers B, Pirottin D, Michaux C, et al. Molecular definition of an allelic series of mutations disrupting the myostatin function and causing double-muscling in cattle. Mamm Genome 1998;9:210-3.

23. Amthor H, Macharia R, Navarrete R, Schuelke M, Brown SC, Otto A, et al. Lack of myostatin results in excessive muscle growth but impaired force generation. Proc Natl Acad Sci USA 2007;104:1835-40.

24. Amthor H, Otto A, Vulin A, Rochat A, Dumonceaux J, Garcia L, et al. Muscle hypertrophy driven by myostatin blockade does not require stem/precursor-cell activity. Proc Natl Acad Sci USA 2009;106:7479-84.

25. Sawitzky M, Zeissler A, Langhammer M, Bielohuby M, Stock P, Hammon HM, et al. Phenotype selection reveals coevolution of muscle glycogen and protein and PTEN as a gate keeper for the accretion of muscle mass in adult female mice. Plos One 2012;7:e39711.

26. Chelh I, Meunier B, Picard B, Reecy MJ, Chevalier C, Hocquette JF, et al. Molecular profiles of Quadriceps muscle in myostatin-null mice reveal PI3K and apoptotic pathways as myostatin targets. BMC Genomics 2009;10:196.

27. Wu M, Falasca M, Blough ER. Akt/protein kinase B in skeletal muscle physiology and pathology. J Cell Physiol 2011;226:29-36.

28. Schiaffino S, Dyar KA, Ciciliot S, Blaauw B, Sandri M. Mechanisms regulating skeletal muscle growth and atrophy. FEBS J 2013;280:4294-314.

29. Hennerby A, Berry C, Siriett V, 0'Callaghan P, Chau L, Watson T, et al. Myostatin regulates fiber-type composition of skeletal muscle by regulating MEF2 and MyoD gene expression. Am J Physiol Cell Physiol 2009;296:C525-34.

30. Dubowitz VC, Sewry A, Oldfors A. Normal muscle, p. 28-55. In: VC Dubowitz, A Sewry and A Oldfors (eds.) Muscle biopsy. A practical approach, 4th ed. Saunders Elsevier; 2013. 


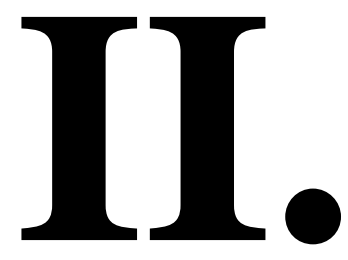




\title{
RESEARCH ARTICLE
}

\section{Myostatin propeptide mutation of the hypermuscular Compact mice decreases the formation of myostatin and improves insulin sensitivity}

\author{
Tamas Kocsis, ${ }^{1}$ Gyorgy Trencsenyi, ${ }^{2}$ Kitti Szabo, ${ }^{1}$ Julia Aliz Baan, ${ }^{1}$ Geza Muller, ${ }^{1}$ Luca Mendler, ${ }^{1}$ \\ Ildiko Garai, ${ }^{2}$ Hans Reinauer, ${ }^{3}$ Ferenc Deak, ${ }^{4}$ Laszlo Dux, ${ }^{1}$ and Aniko Keller-Pinter ${ }^{1}$ \\ ${ }^{1}$ Department of Biochemistry, Faculty of Medicine, University of Szeged, Szeged, Hungary; ${ }^{2}$ Scanomed, Debrecen, Hungary; \\ ${ }^{3}$ INSTAND, Dusseldorf, Germany; and ${ }^{4}$ Institute of Genetics, Biological Research Centre, Hungarian Academy of Sciences, \\ Szeged, Hungary
}

Submitted 8 June 2016; accepted in final form 30 November 2016

Kocsis T, Trencsenyi G, Szabo K, Baan JA, Muller G, Mendler L, Garai I, Reinauer H, Deak F, Dux L, Keller-Pinter A. Myostatin propeptide mutation of the hypermuscular Compact mice decreases the formation of myostatin and improves insulin sensitivity. Am J Physiol Endocrinol Metab 312: E150-E160, 2017. First published December 13, 2016; doi:10.1152/ajpendo.00216.2016.-The TGF $\beta$ family member myostatin (growth/differentiation factor-8) is a negative regulator of skeletal muscle growth. The hypermuscular Compact mice carry the 12-bp $\operatorname{Mstn}(\mathrm{Cmpt}-d l 1 \mathrm{Abc})$ deletion in the sequence encoding the propeptide region of the precursor promyostatin, and additional modifier genes of the Compact genetic background contribute to determine the full expression of the phenotype. In this study, by using mice strains carrying mutant or wild-type myostatin alleles with the Compact genetic background and nonmutant myostatin with the wild-type background, we studied separately the effect of the $M \sin (C m p t-d l 1 A b c)$ mutation or the Compact genetic background on morphology, metabolism, and signaling. We show that both the Compact myostatin mutation and Compact genetic background account for determination of skeletal muscle size. Despite the increased musculature of Compacts, the absolute size of heart and kidney is not influenced by myostatin mutation; however, the Compact genetic background increases them. Both Compact myostatin and genetic background exhibit systemic metabolic effects. The Compact mutation decreases adiposity and improves whole body glucose uptake, insulin sensitivity, and ${ }^{18}$ FDG uptake of skeletal muscle and white adipose tissue, whereas the Compact genetic background has the opposite effect. Importantly, the mutation does not prevent the formation of mature myostatin; however, a decrease in myostatin level was observed, leading to altered activation of Smad2, Smad1/ $5 / 8$, and Akt, and an increased level of p-AS160, a Rab-GTPaseactivating protein responsible for GLUT4 translocation. Based on our analysis, the Compact genetic background strengthens the effect of myostatin mutation on muscle mass, but those can compensate for each other when systemic metabolic effects are compared.

myostatin; Compact mice; skeletal muscle; insulin resistance; 2-deoxy-2- $\left[{ }^{18} \mathrm{~F}\right]$ fluoro-D-glucose

MYOSTATIN [growth/differentiation factor-8 (GDF-8)] is a member of the TGF $\beta$ superfamily and is expressed predominantly in skeletal muscle (31). Myostatin is synthesized as a precursor protein, promyostatin, which undergoes dimerization and proteolytic processing; promyostatin dimer is cleaved by furin

Address for reprint requests and other correspondence: A. Keller-Pinter, Dept. of Biochemistry, Faculty of Medicine, University of Szeged, Dom square 9, H-6720 Szeged, Hungary (e-mail: keller.aniko@med.u-szeged.hu). proteases to $\mathrm{NH}_{2}$-terminal propeptide fragments and $\mathrm{COOH}$ terminal disulfide-linked myostatin dimer (24). However, the propeptides can still associate with myostatin dimer via noncovalent bonds to form a latent complex that sequesters functional myostatin by preventing its binding to the receptor $(24,45)$. Myostatin acts through activin type IIB receptor (ActRIIB) (24), and the signaling involves Smad2/3 transcription factors $(23,57)$; furthermore, it influences the phosphatidylinositol 3-kinase (PI3K)/Akt pathway, which is the key regulator of the anabolic and catabolic responses in skeletal muscle (53).

Myostatin regulates the proliferation and differentiation of myoblasts $(23,46)$; moreover, it also controls the activation and proliferation of satellite cells, the stem cells of skeletal muscle (29). Homozygous disruption of the myostatin gene (31), administration of myostatin propeptide (25), or naturally occurring myostatin gene mutations, e.g., in humans (38), mouse (42), cattle (20), or sheep (10), result in widespread increase of skeletal muscle mass ("double-muscled" phenotype). However, the effect of myostatin is not restricted to skeletal muscle. Beside the autocrine and paracrine effects, it can serve as an endocrine factor. Myostatin was reported to influence the synthesis and secretion of IGF-1 (insulin-like growth factor-1) in the liver, thereby regulating the amount of circulating IGF-1 (51).

Several studies suggest that loss of myostatin or reduction in active myostatin levels leads to increased insulin sensitivity. Myostatin-null mice have increased muscle mass and reduced body fat $(14,26,32)$ and exhibit increased insulin sensitivity (14), which depends on AMP-activated protein kinase (55). Transgenic expression of myostatin propeptide prevents diet-induced obesity and insulin resistance (56), and the overexpression of follistatin-like 3, an inhibitor of members of the TGF $\beta$ family (6), or inhibition of myostatin by dominant-negative myostatin receptor (13) improves insulin sensitivity. Furthermore, increased serum and muscle myostatin levels were shown in insulin-resistant human individuals (17).

The naturally occurring Compact mutation of the myostatin gene arose in a selection program on protein amount and hypermuscularity conducted at the Technical University of Berlin $(7,8)$. Genetic analysis of the Hungarian subpopulation of the hypermuscular Compact mice identified a 12-bp deletion, denoted $M \operatorname{stn}(\mathrm{Cmpt}-\mathrm{dllAbc})$, in the propeptide of the promyostatin (42). The biologically active growth factor do- 
main of myostatin is unaffected by Compact mutation; therefore, the loss of myostatin activity cannot be explained by disruption of the growth factor bioactive domain. However, the mutation can lead to misfolding or defect in secretion and mistargeting of mature myostatin (42). Additional modifier genes should be present to determine the full expression of the Compact phenotype; however, these modifier genes of the special Compact genetic background have not yet been identified (47, 48). Furthermore, the molecular consequences of Compact myostatin mutation, which can regulate muscle size and metabolism, have not been examined. In this study, by using a congenic wild-type mice strain with wild-type myostatin and Compact genetic background, we could separately study the effect of Compact myostatin mutation and genetic background on morphology, metabolism, and signaling. The Compact mice show several similarities compared with myostatin knockout animals; however, numerous alterations exist. The Compact mutation decreased adiposity and improved insulin sensitivity and glucose uptake, whereas the genetic background exhibited the opposite effect. Importantly, here we show that the mature myostatin protein is present in Compact mice, and the 12-bp deletion in the sequence encoding the propeptide decreased the formation of mature myostatin in accordance with increased muscle mass.

\section{MATERIALS AND METHODS}

Animals. The Compact line carrying the 12-bp deletion in the propeptide of promyostatin (Fig. $1 A$ ) was selected and inbred in a long-term selection experiment in Berlin, Germany $(8,50)$. The origin of the Hungarian subpopulation of the Compact line was described earlier $(3,22)$. The BALB/c mice carrying wild-type myostatin were obtained from the Biological Research Centre of the Hungarian Academy of Sciences (Szeged, Hungary). The Compact mice were crossed with BALB/c to introgress the wildtype myostatin gene of BALB/c to Compact mice. The wild-type myostatin allele was followed through five generations of repeated backcrossing with the Compact line. Heterozygous animals of this line in generation B5 were mated inter se to produce homozygous wild-type animals with a Compact genetic background (denoted as congenic wild-type animals). Genotyping for Compact myostatin mutation [Mstn(Cmpt-dllAbc)] was described earlier (42). Since the Compact hypermuscular phenotype is stronger in males than females (47), we performed the study on male 3- to 4-mo-old and 10 -mo-old mice. Animal experiments conformed to the National Institutes of Health's Guide for the Care and Use of Laboratory
A

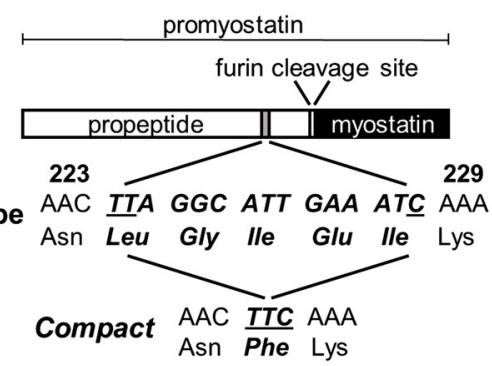

C

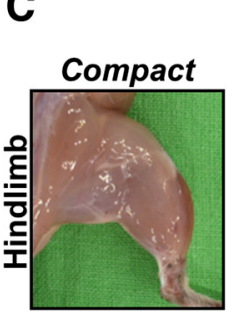

D

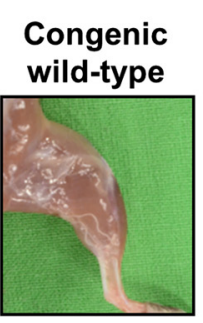

$B A L B / C$

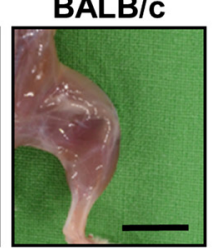

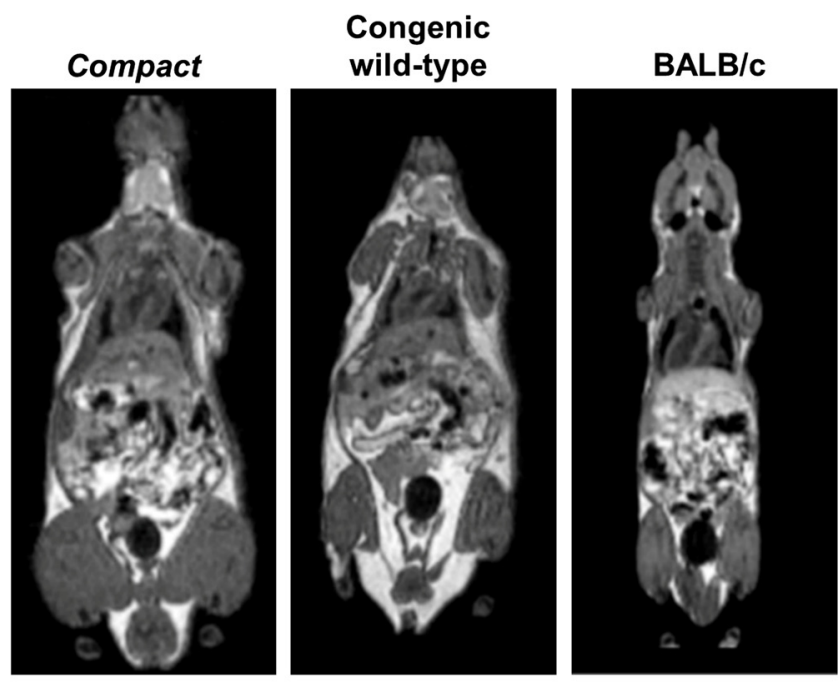

B

Compact $\square$ Congenic wild-type

BALB/C
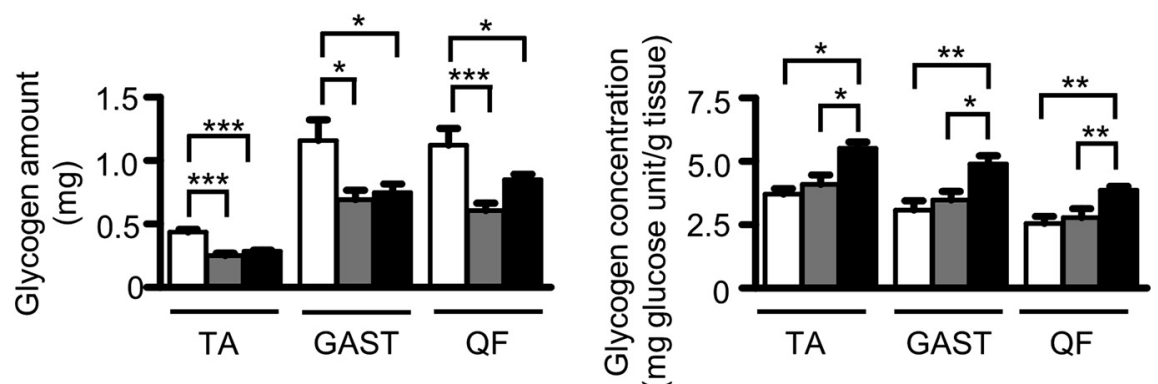

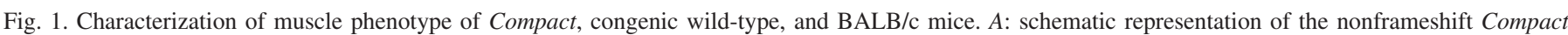

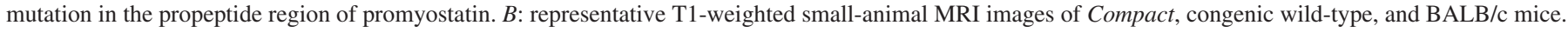

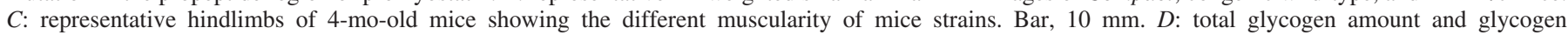

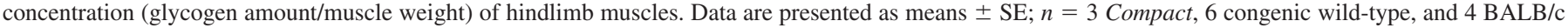

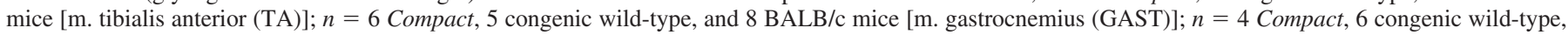
and $9 \mathrm{BALB} / \mathrm{c}$ mice $[\mathrm{m}$. quadriceps femoris $(\mathrm{QF})]$. $* P<0.05 ; * * P<0.01 ; * * * P<0.001$. 
Animals (NIH Publication No. 85-23, revised 1996) and were approved by the local Ethics Committee at the University of Szeged.

Determination of tissue glycogen content. Nonfasting 3- to 4-moold male mice were euthanized, and tissues were frozen in liquid nitrogen. Total glycogen amount of the tissue samples was measured as glucose residues by hexokinase/glucose-6-phosphate dehydrogenase assay (Roche) following acidic hydrolysis, as described earlier (22).

Periodic acid Schiff staining. Glycogen content of the liver samples was visualized on 5 - $\mu$ m-thin cryosections by periodic acid Schiff (PAS) staining, as described previously (22).

Measurement of tissue alanine aminotransferase activity. Liver samples of 3- to 4-mo-old male mice were frozen in liquid nitrogen, and tissue homogenates were prepared as described at Western blotting. The activity of alanine aminotransferase (ALT) enzyme of the samples was determined by lactate dehydrogenase-coupled kinetic colorimetric assay (Diagnosticum, Budapest, Hungary) in accordance with the manufacturer's instructions. Spectrophotometry was performed with Fluostar Optima (BMG Labtech, Ortenberg, Germany).

Western blotting. To analyze protein expression levels, $\mathrm{m}$. gastrocnemius and liver samples of 3- to 4-mo-old male animals were homogenized at $4^{\circ} \mathrm{C}$ in a buffer $[50 \mathrm{mM}$ Tris $\cdot \mathrm{HCl}(\mathrm{pH} 7.6), 100 \mathrm{mM}$ $\mathrm{NaCl}$, and $10 \mathrm{mM}$ EDTA] supplemented with protease inhibitor cocktail (Sigma-Aldrich), $1 \mathrm{mM}$ natrium-orthovanadate, and $1 \mathrm{mM}$ natrium-fluoride. Following centrifugation at $4{ }^{\circ} \mathrm{C}$ for $10 \mathrm{~min}$ at 11,000 $g$ (Hettich Universal 320R, DJB; Labcare, Buckinghamshire, UK) to remove cellular debris, the protein concentration of the supernatants was determined by BCA kit (Thermo Scientific). The samples were separated on $10 \%$ SDS-polyacrylamide gel under reducing or nonreducing conditions and transferred onto Protran nitrocellulose membrane (Amersham, GE Healthcare). After incubation in $5 \%$ blocking agent (Bio-Rad), the membrane was incubated with anti-phospho-Akt Ser ${ }^{473}$ (no. 4051; Cell Signaling Technology), antiphospho-Smad1/5/8 Ser ${ }^{463 / 465}$ (no. 9511; Cell Signaling Technology), anti-phospho-Smad2 Ser ${ }^{465 / 467}$ (44-244G; Invitrogen), anti-Smad4 (sc-7966; Santa Cruz Biotechnology), anti-Akt (no. 4691; Cell Signaling Technology), anti-myostatin (AB3239-I; Millipore), antiGDF8 propeptide (MAB7881; R\&D Systems), anti-phospho-AS160 (no. 8881S; Cell Signaling Technology), anti-GLUT4 (no. 2213S; Cell Signaling Technology), or anti-GAPDH (no. 2118; Cell Signaling Technology) primary antibodies, followed by incubation with appropriate horseradish peroxidase-conjugated anti-IgG secondary antibody [P0448 and P0161 (DAKO), 112-035-003 (Jackson Immunoresearch)]. Mouse recombinant myostatin (788-G8; R\&D Systems) was used as a positive control. ECL reagent (Advansta, Menlo Park, CA) was used for substrate detection, and the membrane was exposed to X-ray film (AGFA) for visualization.

Intraperitoneal pyruvate tolerance test. Three- to four-month-old male mice were fasted for $16 \mathrm{~h}$ before intraperitoneal pyruvate tolerance test and had free access to water. Following the measurement of baseline blood glucose levels, mice were injected with intraperitoneal pyruvate ( $2 \mathrm{mg}$ pyruvate/ $1 \mathrm{~g}$ body $\mathrm{wt}$ ). Blood glucose was measured from distal tail vein at 15, 30, 45, 60, 90, and 120 min. All blood glucose measurements were performed by the AccuCheck blood glucose monitoring system (Roche).

Intraperitoneal glucose and insulin tolerance tests. For intraperitoneal glucose tolerance test, male mice were fasted for $16 \mathrm{~h}$ and had free access to water. The measurement of baseline blood glucose was followed by intraperitoneal injection of D-glucose $(2 \mathrm{mg}$ glucose $/ 1 \mathrm{~g}$ body wt), and blood glucose was determined from distal tail vein at 30, 60, 90, and $120 \mathrm{~min}$.

For intraperitoneal insulin tolerance test, animals were fasted for 5 $\mathrm{h}$ and had free access to water, and their baseline blood glucose was measured. After the injection of intraperitoneal insulin bolus (1.0 U/1 kg body wt, Humulin R; Eli Lilly, Grootslag, The Netherlands), blood glucose was measured from the distal tail vein at 15, 30, 45, 60, 90, and $120 \mathrm{~min}$. Blood glucose measurements were performed by the AccuCheck blood glucose monitoring system (Roche).

Small-animal PET/MRI imaging using 2-deoxy-2-[18 F]fluoro-Dglucose. Ten-month-old male mice were injected with $7.0 \pm 0.2 \mathrm{MBq}$ of ${ }^{18}$ FDG (2-deoxy-2- $\left[{ }^{18} \mathrm{~F}\right]$ fluoro-D-glucose) via the lateral tail vein in $0.2-\mathrm{ml}$ volume. Fifty minutes after ${ }^{18}$ FDG injection, the animals were anesthetized by $3 \%$ isoflurane with a dedicated small-animal anesthesia device, and whole body PET scans (10-min static PET scans) were acquired using the preclinical nanoScan PET/MRI system (Mediso). To prevent movement, animals were fixed to a mouse chamber (MultiCell Imaging Chamber; Mediso) and positioned in the center of the field of view (FOV). For the determination of the anatomic localization of the organs and tissues, T1-weighted MRI scans were performed (3D GRE EXT multi-FOV, TR/TE 15/2 ms, FOV $70 \mathrm{~mm}$, NEX 2). PET volumes were reconstructed using a three-dimensional Ordered Subsets Expectation Maximization (3D-OSEM) algorithm (Tera-Tomo; Mediso). PET and MRI images were automatically coregistered by the PET/MRI instrument's acquisition software $(\mathrm{Nu}-$ cline). Reconstructed, reoriented, and coregistered images were further analyzed with InterView FUSION (Mediso) dedicated image analysis software. Radiotracer uptake was expressed in terms of standardized uptake values (SUVs). Ellipsoidal three-dimensional volumes of interest (VOI) were manually drawn around the edge of the tissue or organ activity by visual inspection using InterView FUSION multimodal visualization and evaluation software (Mediso). The standardized uptake value (SUV) was calculated as follows: SUV $=[$ VOI activity $(\mathrm{Bq} / \mathrm{ml})] /[$ injected activity $(\mathrm{Bq}) /$ animal weight (g)], assuming a density of $1 \mathrm{~g} / \mathrm{ml}$. SUV mean is the average SUV value within the volume of interest (VOI).

Statistical analysis. Statistical evaluations were performed by 1-way ANOVA and Newman-Keuls posttest (GraphPad Software). All data are presented as means $\pm \mathrm{SE}$.

\section{RESULTS}

Body composition of Compact mice. The body weights of the congenic wild-type mice carrying the wild-type myostatin gene in Compact genetic background were higher than those of $\mathrm{BALB} / \mathrm{c}$ mice, but they were smaller than the Compact animals (Table 1). We showed by MRI analysis that profound differences exist in body composition between the genotypes. The qualitative analysis of T1-weighted MRI images revealed remarkable enlargement of skeletal muscle tissues in Compact mice. In the MRI images of congenic wild-type animals, the spaciousness of fat tissues was clearly visualized (Fig. 1B). The gross enhancement of hindlimb muscle mass was observable (Fig. 1C). The absolute weights of individual hindlimb muscles such as tibialis anterior, quadriceps femoris, and gastrocnemius muscles were almost two times greater in Compacts compared with congenic wild-type animals, and they were bigger in congenic wild-type animals than in BALB/c mice (Table 1). The muscle weight/body weight ratios showed the highest values in Compacts and the lowest in congenic wild-type mice (Table 1).

We found that the absolute weights of heart and kidney of the Compact and congenic wild-type mice were comparable and higher than that of BALB/c mice. The absolute weight of abdominal fat increased by $\sim 30 \%$ in 3 - to 4 -mo-old mice and $\sim 50 \%$ in 10-mo-old congenic wild-type compared with Compacts mice, and it was markedly lower in the BALB/c group than in Compacts (Table 2). Liver/body weight ratio of Compacts was smaller than that of congenic wild-type animals in both ages (Table 2). Abdominal fat/body weight ratios were comparable in Compact and BALB/c animals, and it was 
Table 1. Absolute and normalized muscle weights

\begin{tabular}{|c|c|c|c|}
\hline & Compact & Congenic wild-type & $\mathrm{BALB} / \mathrm{c}$ \\
\hline & \multicolumn{3}{|c|}{ 3-to 4-Mo-old animals } \\
\hline Body weight, g & $47.4 \pm 0.67^{\mathrm{b}, \mathrm{e}}$ & $40.8 \pm 1.76^{\mathrm{e}}$ & $25.9 \pm 0.48$ \\
\hline \multicolumn{4}{|l|}{ Muscle weight, mg } \\
\hline TA & $113.8 \pm 1.64^{\mathrm{b}, \mathrm{e}}$ & $67.6 \pm 4.70^{\mathrm{e}}$ & $45.9 \pm 0.88$ \\
\hline GAST & $350.3 \pm 8.15^{\mathrm{b}, \mathrm{e}}$ & $183.9 \pm 5.81^{\mathrm{e}}$ & $140.5 \pm 2.14$ \\
\hline QF & $470.3 \pm 12.81^{\mathrm{b}, \mathrm{e}}$ & $246.1 \pm 6.71^{\mathrm{d}}$ & $204.6 \pm 3.65$ \\
\hline \multicolumn{4}{|c|}{ Muscle weight/body weight, mg/g } \\
\hline TA/body weight & $2.4 \pm 0.04^{\mathrm{b}, \mathrm{e}}$ & $1.6 \pm 0.05^{\mathrm{c}}$ & $1.8 \pm 0.04$ \\
\hline GAST/body weight & $7.4 \pm 0.13^{\mathrm{b}, \mathrm{e}}$ & $4.5 \pm 0.11^{\mathrm{e}}$ & $5.4 \pm 0.08$ \\
\hline \multirow[t]{2}{*}{$\mathrm{QF} / \mathrm{bw}$} & $9.9 \pm 0.22^{\mathrm{b}, \mathrm{e}}$ & $6.1 \pm 0.22^{\mathrm{e}}$ & $7.9 \pm 0.11$ \\
\hline & \multicolumn{2}{|c|}{10 Mo old animals } & \\
\hline Body weight, g & $50.4 \pm 0.53^{\mathrm{a}, \mathrm{e}}$ & $45.1 \pm 1.29^{\mathrm{e}}$ & $31.4 \pm 0.88$ \\
\hline \multicolumn{4}{|l|}{ Muscle weight, mg } \\
\hline TA & $141.8 \pm 7.18^{\mathrm{b}, \mathrm{e}}$ & $69.5 \pm 2.45^{\mathrm{c}}$ & $54.3 \pm 2.45$ \\
\hline GAST & $385.4 \pm 10.64^{\mathrm{b}, \mathrm{e}}$ & $185.0 \pm 2.45^{\mathrm{c}}$ & $160.5 \pm 6.52$ \\
\hline QF & $476.0 \pm 14.09^{\mathrm{b}, \mathrm{e}}$ & $247.5 \pm 4.78$ & $221.6 \pm 9.43$ \\
\hline \multicolumn{4}{|c|}{ Muscle weight/body weight, mg/g } \\
\hline TA/body weight & $2.8 \pm 0.14^{\mathrm{b}, \mathrm{e}}$ & $1.56 \pm 0.08$ & $1.7 \pm 0.07$ \\
\hline GAST/body weight & $7.6 \pm 0.1^{\mathrm{b}, \mathrm{e}}$ & $4.13 \pm 0.09^{\mathrm{e}}$ & $5.1 \pm 0.11$ \\
\hline QF/body weight & $9.4 \pm 0.22^{\mathrm{b}, \mathrm{e}}$ & $5.52 \pm 0.12^{\mathrm{e}}$ & $7.0 \pm 0.12$ \\
\hline
\end{tabular}

Values are means $\pm \mathrm{SE}$ (3- to 4-mo-old animals: $n=9$ Compact, 7 congenic wild-type, and $8 \mathrm{BALB} / \mathrm{c}$ mice; 10-mo-old animals: $n=8$ Compact, 12 congenic wild-type, and $8 \mathrm{BALB} / \mathrm{c}$ mice). TA, m. tibialis anterior; GAST, m. gastrocnemius; QF, m. quadriceps femoris. The values of the Compact group are significantly different from the congenic wild-type group: ${ }^{\text {a }} P<0.01$; ${ }^{\mathrm{b}} P<0.001$. The values of the Compact or congenic wild-type group are significantly different from the BALB/c group: ${ }^{\mathrm{c}} P<0.05$; ${ }^{\mathrm{d}} P<0.01$; ${ }^{\mathrm{e}} P<$ 0.001 .

almost twofold higher in congenic wild-type mice (Table 2). The weights of heart and kidney in proportion to body weight were the smallest in Compacts and highest in BALB/c mice (Table 2).

Glycogen accumulation is determined by the genetic background of Compact mice. Myostatin was reported to influence the glycogen content of $\mathrm{C} 2 \mathrm{C} 12$ myoblasts (9), and muscle glycogen was reduced in type 2 diabetes mellitus (15). Previously, we have shown that Compact tibialis anterior muscle contains more glycogen than that of BALB/c (22). To distinguish the role of Compact mutation and the Compact genetic background in the regulation of glycogen stores, here we compared the glycogen content of Compact, congenic wildtype, and BALB/c muscles. We found that total glycogen levels of Compact muscles were the highest, and the congenic wild-type and BALB/c samples contained comparable and smaller amounts of glycogen (Fig. 1D). However, the glycogen concentration was the highest in BALB/c mice and displayed no differences between Compact and congenic wild-type groups (Fig. 1D).

Both the myostatin mutation and Compact genetic background influenced liver weight, and liver functions as a glycogen store; therefore, we measured the glycogen content of the liver samples. Analyzing the total glycogen amount and glycogen concentration, we did not observe differences between congenic wild-type and Compact groups; the glycogen content/liver weight ratio of BALB/c animals was $>2.5$-fold smaller (Fig. $2 A$ ). The visualization of glycogen by PAS staining verified the results of spectrophotometry, and weaker staining was observed in BALB/c samples (Fig. 2B).

Liver characteristics in Compact mice. It was reported that knocking out of myostatin results in comparable absolute liver weight, lower liver/body weight ratio, and decreased ALT activity of the liver (18). We found that liver mass and liver/body weight ratios are not increased in proportion to skeletal muscle mass in Compact mice; therefore, we aimed to assess whether ALT activity in proportion to body weight is also reduced similarly to myostatin knockout animals. Our data show that total liver ALT activity/body weight ratio was the lowest in Compacts and the biggest in BALB/c mice (Table 3).

Table 2. Absolute and normalized organ weights

\begin{tabular}{|c|c|c|c|}
\hline & Compact & Congenic wild-type & $\mathrm{BALB} / \mathrm{c}$ \\
\hline & & to 4-Mo-old animals & \\
\hline \multicolumn{4}{|l|}{ Organ weight, mg } \\
\hline Liver & $1,792.0 \pm 43.45^{\mathrm{e}}$ & $1,874.0 \pm 33.68^{\mathrm{e}}$ & $1,158.0 \pm 31.55$ \\
\hline Abdominal fat & $672.8 \pm 59.07^{\mathrm{b}, \mathrm{d}}$ & $1,042.0 \pm 105.30^{\mathrm{e}}$ & $271.9 \pm 20.21$ \\
\hline Heart & $153.8 \pm 4.71^{\mathrm{d}}$ & $163.1 \pm 11.41^{\mathrm{d}}$ & $121.4 \pm 3.17$ \\
\hline Kidney & $237.8 \pm 5.97^{\mathrm{e}}$ & $250.6 \pm 11.76^{\mathrm{e}}$ & $190.4 \pm 5.42$ \\
\hline \multicolumn{4}{|l|}{ Organ weight/body weight, $\mathrm{mg} / \mathrm{g}$} \\
\hline Liver/body weight & $37.8 \pm 0.81^{\mathrm{c}, \mathrm{e}}$ & $46.3 \pm 1.41$ & $44.72 \pm 1.04$ \\
\hline Abdominal fat/body weight & $14.5 \pm 1.30^{\mathrm{c}}$ & $25.3 \pm 1.76^{\mathrm{e}}$ & $10.55 \pm 0.89$ \\
\hline Heart/body weight & $3.3 \pm 0.13^{\mathrm{c}, \mathrm{e}}$ & $3.9 \pm 0.12^{\mathrm{e}}$ & $4.68 \pm 0.08$ \\
\hline \multirow[t]{2}{*}{ Kidney/body weight } & $5.0 \pm 0.11^{\mathrm{c}, \mathrm{e}}$ & $6.16 \pm 0.17^{\mathrm{e}}$ & $7.35 \pm 0.17$ \\
\hline & & 10-Mo-old animals & \\
\hline \multicolumn{4}{|l|}{ Organ weight, mg } \\
\hline Liver & $2,037.0 \pm 43.15^{\mathrm{a}, \mathrm{e}}$ & $2,213.0 \pm 65.05^{\mathrm{e}}$ & $1,525.0 \pm 49.64$ \\
\hline Abdominal fat & $683.5 \pm 35.04^{\mathrm{b}}$ & $1,236.0 \pm 156.3^{\mathrm{e}}$ & $374.6 \pm 41.99$ \\
\hline Heart & $173.0 \pm 3.21^{\mathrm{e}}$ & $178.2 \pm 5.67^{\mathrm{e}}$ & $140.3 \pm 5.11$ \\
\hline Kidney & $266.9 \pm 3.63$ & $300.3 \pm 12.03$ & $290.6 \pm 21.00$ \\
\hline \multicolumn{4}{|l|}{ Organ weight/body weight, $\mathrm{mg} / \mathrm{g}$} \\
\hline Liver/body weight & $40.4 \pm 0.80^{\mathrm{c}, \mathrm{e}}$ & $49.3 \pm 1.29$ & $48.6 \pm 0.82$ \\
\hline Abdominal fat/body weight & $13.6 \pm 0.75^{\mathrm{c}}$ & $26.8 \pm 2.68^{\mathrm{e}}$ & $12.1 \pm 1.48$ \\
\hline Heart/body weight & $3.4 \pm 0.07^{\mathrm{c}, \mathrm{e}}$ & $3.9 \pm 0.07^{\mathrm{e}}$ & $4.5 \pm 0.11$ \\
\hline Kidney/body weight & $5.3 \pm 0.10^{\mathrm{a}, \mathrm{e}}$ & $6.6 \pm 0.11^{\mathrm{e}}$ & $9.3 \pm 0.73$ \\
\hline
\end{tabular}

Values are means \pm SE (3- to 4-mo-old animals: $n=9$ Compact, 7 congenic wild-type, and 8 BALB/c mice; 10-mo-old animals: $n=8$ Compact, 12 congenic wild-type, and $8 \mathrm{BALB} / \mathrm{c}$ mice). The values of Compact group are significantly different from congenic wild-type group: ${ }^{\mathrm{a}} P<0.05$; ${ }^{\mathrm{b}} P<0.01 ;{ }^{\mathrm{c}} P<0.001$. The values of Compact or congenic wild-type group are significantly different from the BALB/c group: ${ }^{\mathrm{d}} P<0.01 ;{ }^{\mathrm{e}} P<0.001$. 
A
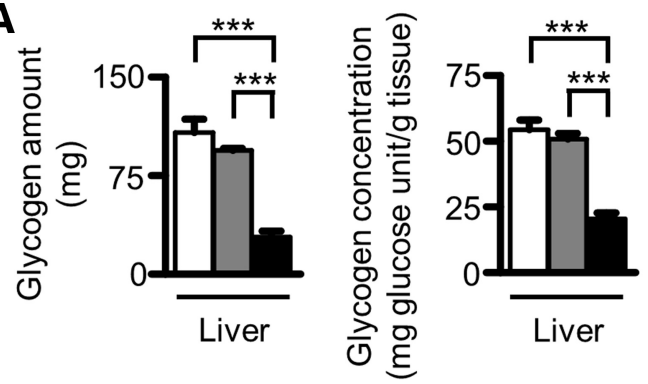

B

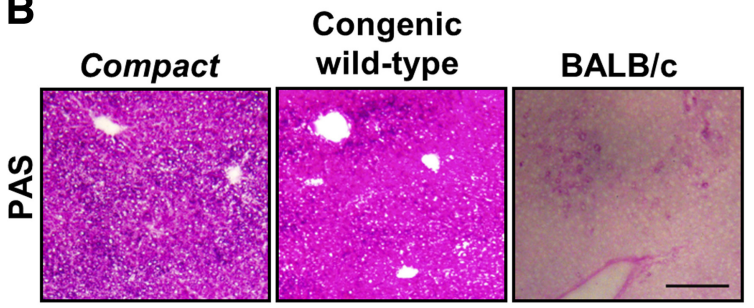

C

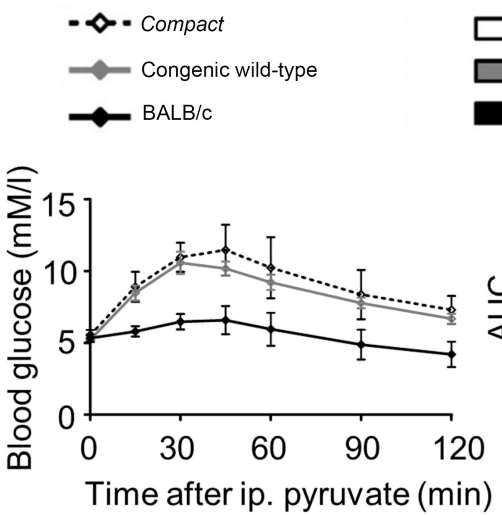

Congenic wild-type

$B A L B / C$

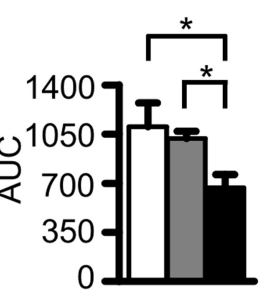

Fig. 2. Characterization of the Compact, congenic wild-type, and BALB/c livers. A: total glycogen amount and glycogen concentration of the liver samples determined by spectrophotometry $(n=4$ Compact, 5 congenic wild-type, and $4 \mathrm{BALB} / \mathrm{c}$ mice). $B$ : representative periodic acid Schiff (PAS)stained images show glycogen content of the liver. Scale bar, $200 \mu \mathrm{m}$. $C$ : intraperitoneal (ip) pyruvate tolerance test of 3- to 4-mo-old mice $(n=3$ Compact, 5 congenic wild-type, and $4 \mathrm{BALB} / \mathrm{c}$ mice). Area under the curve (AUC) values are presented in bar diagrams. Data are reported as means $\pm \mathrm{SE}$. $* P<0.05 ; * * * P<0.001$.

Next, we administered glucose precursor pyruvate to measure hepatic gluconeogenesis. The intraperitoneal pyruvate tolerance test showed that hepatic gluconeogenesis increased in Compact and congenic wild-type animals compared with BALB/c animals. The area under the curve of blood glucose concentrations during pyruvate tolerance test was comparable in Compact and congenic wild-type mice, and it was lower in BALB/c animals (Fig. 2C).

The Compact mutation of myostatin propeptide decreases myostatin formation. The Compact Mstn (Cmpt-dllAbc) mutation in the myostatin gene eliminates amino acids $224-228$ in the propeptide and creates a new Phe residue (42). The Compact mutation is toward the $\mathrm{NH}_{2}$ terminus from the furin cleavage site, since promyostatin is proteolytically processed by furin at the RSRR (263-266) site to give the active processed myostatin (46). The Compact mutation is a nonframeshift 12-bp deletion, and the biologically active growth factor domain is intact; therefore, functional myostatin formation

should be permitted. The presence of Compact mutation was verified by sequencing the myostatin gene (data not shown). Importantly, we were able to detect mature myostatin dimer in Compact skeletal muscle (Fig. 3). Western blot analysis showed that the level of mature myostatin dimer was the lowest in Compact muscle and highest in BALB/c mice (Fig. 3) in accordance with the skeletal muscle weights of the animals. The anti-propeptide antibody could recognize the mutant propeptide, and the expression level of propeptide was proportional to myostatin level when the genotypes were compared; the lowest amount was detected in Compact samples (Fig. 3).

Effect of Compact myostatin mutation and genetic background on signaling. Myostatin is expressed and secreted predominantly by skeletal muscle; however, as an endocrine factor, it can influence the signal transduction of liver (51). We could detect the mature myostatin; therefore, we investigated myostatin signaling in both skeletal muscle and liver. Despite low levels of myostatin protein in Compact skeletal muscle, the level of phospo-Smad2 was the highest when the genotypes were compared (Fig. 4B), suggesting the potential role of other TGF $\beta$ members in Smad2 activation. The specificity of the anti-p-Smad2 antibody is shown in Fig. 4A. Phospho-Smad2 levels of the liver samples were the lowest in BALB/c mice and the highest in Compacts (Fig. 4C).

Both the Smad2/3 signaling mediated by TGF $\beta$ family members and the Smad1/5/8 pathway mediated by bone morphogenic proteins (BMPs) converge on the common mediator Smad4. The balance between these competing pathways is required to maintain muscle mass; the BMP-mediated Smad1/ $5 / 8$ pathway is the fundamental hypertrophic signal in mice, which is dominant over myostatin signaling, and Smad4 deficiency induces muscle atrophy (37). Furthermore, Smad1/5/8 signaling is an important regulator of liver homeostasis (11, 41). The level of phospho-Smad1/5/8 was significantly higher in muscles of Compacts compared with congenic wild-type samples (Fig. 4B). However, we have not found any significant differences in phospho-Smad1/5/8 levels of liver samples between the genotypes (Fig. 4C). Furthermore, no differences were observed between either muscular (Fig. 4B) or hepatic (Fig. 4C) Smad4 levels.

Myostatin was reported to inhibit the PI3K/Akt pathway (53); therefore, we determined the phosphorylation level of Akt. The phospho-Akt Ser ${ }^{473} / \mathrm{Akt}$ ratio of congenic wild-type liver was lower than that of Compact and comparable with

Table 3. Fasting blood glucose levels and hepatic ALT activity

\begin{tabular}{llll}
\hline \hline & \multicolumn{1}{c}{ Compact } & Congenic wild-type & BALB/c \\
\hline Fasting blood glucose, mM/1 & & \\
3- to 4-Mo-old animals & $5.81 \pm 0.67$ & $5.81 \pm 0.64$ & $5.38 \pm 0.69$ \\
10-Mo-old animals & $5.20 \pm 0.25$ & $5.07 \pm 0.16$ & $4.82 \pm 0.28$ \\
Total hepatic ALT activity.g body wt ${ }^{-1}, \mathrm{U} / \mathrm{g}$ & & \\
3- to 4-mo-old animals & $0.12 \pm 0.008 * \ddagger$ & $0.15 \pm 0.006 \dagger$ & $0.18 \pm 0.002$ \\
\hline
\end{tabular}

Values are means \pm SE [3- to 4-mo-old animals: $n=3$ Compact, 8 congenic wild-type, and 3 BALB/c mice; 10-mo-old animals: $n=5$ Compact, 3 congenic wild-type, and $6 \mathrm{BALB} / \mathrm{c}$ mice for the fasting blood glucose; $n=$ 4 in each group for alanine aminotransferase (ALT) activity]. The values of Compact group are significantly different from congenic wild-type group: ${ }^{*} P<0.05$. The values of Compact or congenic wild-type group are significantly different from the BALB/c group: $\dagger P<0.01 ; \ddagger P<0.001$. 


\section{Recombinant \\ myostatin}

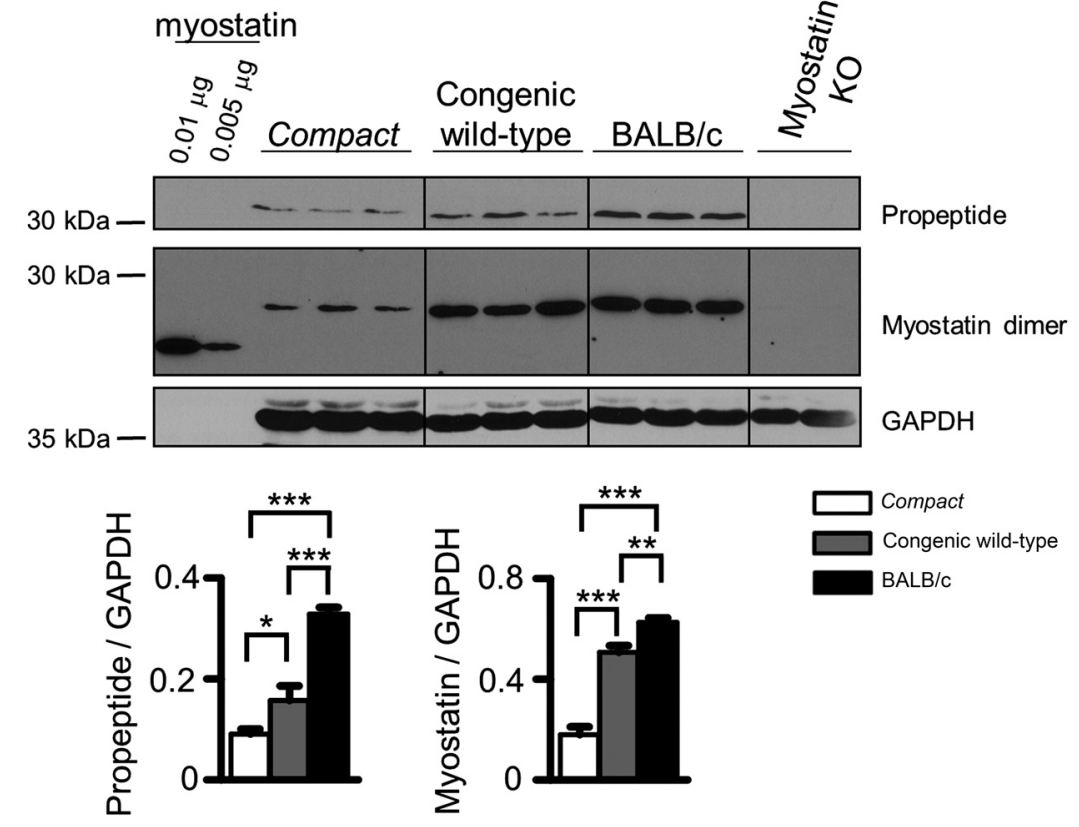

Fig. 3. Myostatin level in skeletal muscle of Compact, congenic wild-type, and BALB/c mice. M. gastrocnemius protein extracts were subjected to SDS-PAGE and blotted with anti-myostatin or anti-propeptide antibody. Representative images are shown. Note the presence of mature myostatin dimer and myostatin propeptide in Compact samples. Mouse recombinant myostatin was used as a positive control, and muscle homogenates of myostatin knockout (KO) mice served as a negative control. Differences in glycosylation may cause altered electrophoretic mobility. Bar diagrams show the quantification of the results. Data are reported as means $\pm \mathrm{SE} ; n=5$ Compact, 5 congenic wild-type, and $6 \mathrm{BALB} / \mathrm{c}$ mice. ${ }^{*} P<0.05$; $* * P<0.01$; $* * * P<0.001$.

BALB/c values (Fig. 4C). In contrast, phospho-Akt Ser ${ }^{473} / \mathrm{Akt}$ ratios were higher in Compact and congenic wild-type muscles compared with BALB/c samples, in accord with myostatin levels (Fig. 4B).

AS160 (Akt substrate of $160 \mathrm{kDa}$ ), a Rab GTPase-activating protein, can regulate the translocation of GLUT4 glucose transporter to the plasma membrane of insulin-sensitive cells (21). The level of phospho-AS160 was lower in congenic wild-type muscle samples compared with those of Compact or BALB/c mice (Fig. 4D). The GLUT4 expression showed equal levels in Compact and congenic wild-type animals, and the level was lower in BALB/c samples (Fig. 4D).

Glucose tolerance and insulin sensitivity are improved by Compact myostatin mutation and reduced by Compact genetic background. As reported previously, knocking out of myostatin increases glucose tolerance and insulin sensitivity $(14,55)$, and fed and fasting glucose levels in myostatin knockout mice were not significantly different from controls (32). Since the Compact mice were hypermuscular and had reduced abdominal fat, we examined whether Compact mice showed alterations in glucose metabolism. Because age is reported to influence glucose tolerance $(2,28)$, we compared whole body glucose tolerance and insulin sensitivity from both young (3- to 4-mo-old) and middle-aged (10-mo-old) groups of mice. Our results did not show any significant changes in fasting blood glucose levels comparing Compact mice with age-matched congenic wild-type and BALB/c animals (Table 3); however, the response to exogenous glucose revealed differences between genotypes. Compact and BALB/c mice showed greater glucose tolerance compared with age-matched congenic wildtype strain. The area under the curve of blood glucose concentrations during the glucose tolerance test was significantly higher in congenic wild-type mice compared with age-matched Compact and BALB/c (Fig. 5A). We performed insulin tolerance tests to measure blood glucose changes following insulin administration. Insulin treatment reduced blood glucose levels in all groups of mice, indicating the insulin responsivity.
Congenic wild-type mice showed weaker insulin sensitivity compared with Compact and BALB/c groups. The area under the curve value during the insulin tolerance test was significantly higher in congenic wild-types than in Compact and BALB/c mice at both ages (Fig. 5, $C$ and $D$ ). Both glucose tolerance and insulin sensitivity tests showed comparable results between age-matched Compacts and BALB/c mice (Fig. $5, A-D)$.

To test the effect of aging on glucose tolerance and insulin sensitivity, the area under the curve values were compared. The area under the curve of blood glucose concentrations during glucose tolerance tests of 10-mo-old Compacts was significantly higher than that of 3- to 4-moold animals $(1,154 \pm 31.5$ vs. $943.3 \pm 86.3, P=0.0318)$; no alterations were observed comparing young and middleaged congenic wild-type or BALB/c groups. The area under the curve values during insulin tolerance tests of young animals were not significantly different from middle-aged groups, although they tended to be smaller in all three genotypes.

Compact mutation increases ${ }^{18}$ FDG uptake in skeletal muscle, liver, and adipose tissue. The Compact mutation and genetic background affected glucose tolerance and insulin sensitivity; therefore, we evaluated glucose uptake in different tissues with known insulin responsiveness using small-animal PET/MRI imaging. By the quantitative analysis of decay-corrected ${ }^{18}$ FDG-PET images, we found significant differences in the SUV mean of the selected organs 50 min after tracer injection (Fig. 6). The ${ }^{18}$ FDG accumulation of the skeletal muscle was comparable in Compacts and $\mathrm{BALB} / \mathrm{c}$ animals, and moderate uptake was observed in congenic wild-type animals. The radiotracer uptake of white adipose tissue showed similar results as skeletal muscle, mild uptake was observed in the congenic wild-type mice, and it was approximately twofold higher in Compact and BALB/c animals. The liver of Compact mice showed the 
A

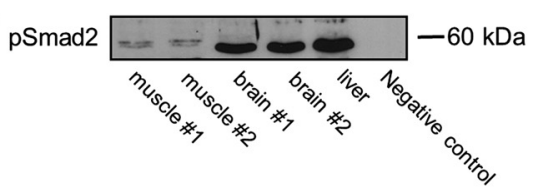

B

\begin{tabular}{c} 
Skeletal muscle \\
\hline Congenic \\
Compact wild-type BALB/C
\end{tabular}
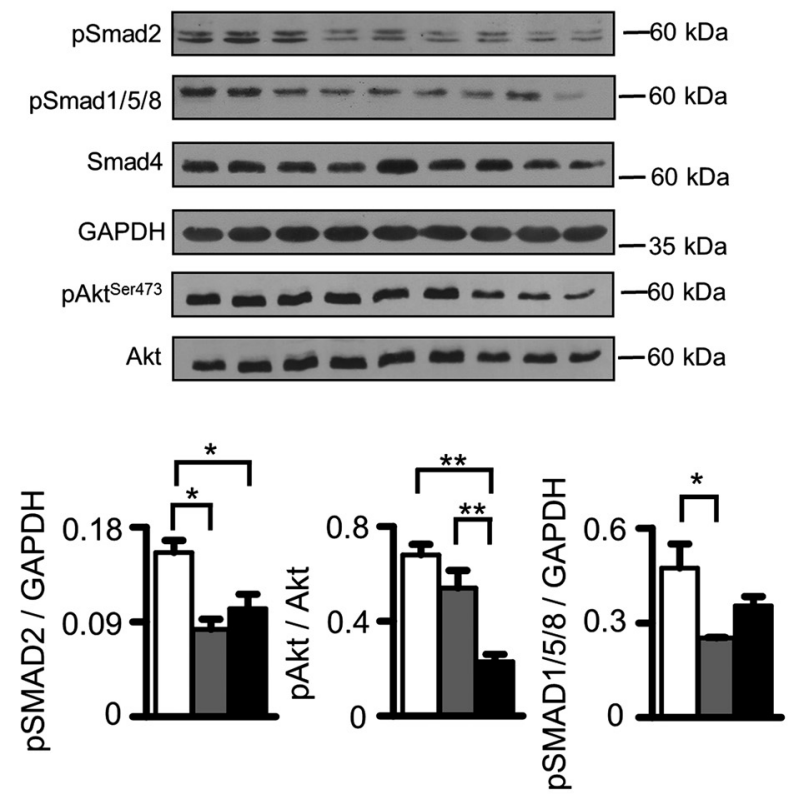

D

Skeletal muscle Congenic Compact wild-type BALB/C

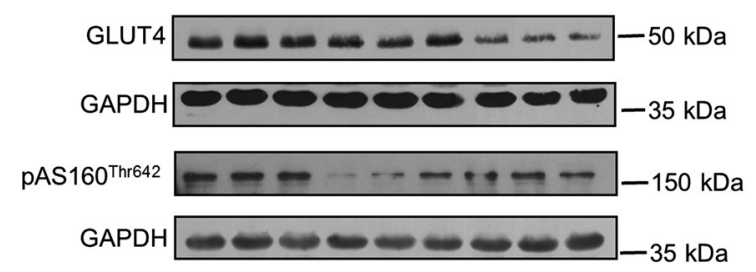

C

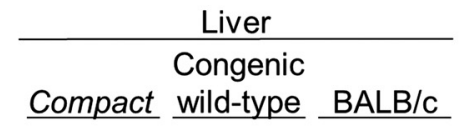

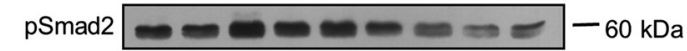

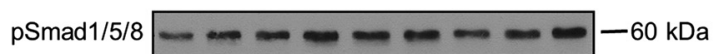

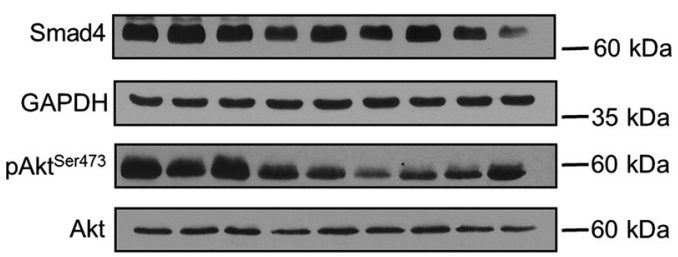

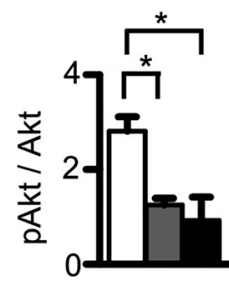

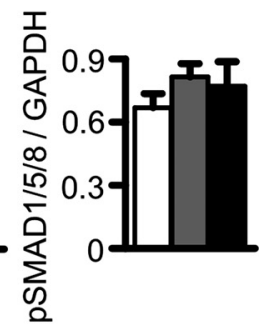

Compact

$\square$ Congenic wild-type

BALB/c

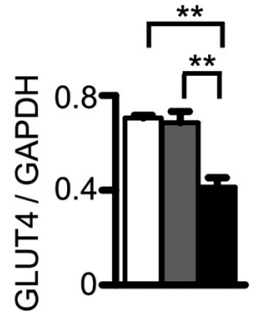

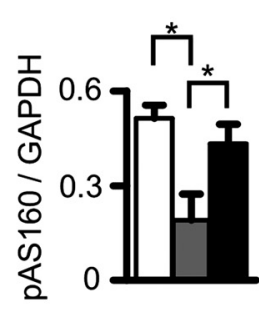

Fig. 4. Alterations of phospho (p)-Smad2, p-Smad1/5/8, p-Akt, and p-AS160 (Akt substrate of $160 \mathrm{kDa}$ ) signaling in the different mouse strains. A: m. gastrocnemius homogenates $(50 \mu \mathrm{g}$ protein/lane), mouse brain $(40 \mu \mathrm{g}$ protein/lane), and mouse liver (60 $\mu \mathrm{g}$ protein/lane) samples of Compact mice were subjected to SDS-PAGE and developed by anti-p-Smad2 antibody to establish the specificity of the antibody. Brain samples served as a positive control, and the negative control was incubated with only the secondary antibody. p-Smad2 was detected at the predicted molecular weight in either mouse skeletal muscle, liver, or positive control samples. Note the low level of p-Smad2 in skeletal muscle compared with liver and brain tissues. $B-D$ : Western blot experiments of Compact, congenic wild-type, and BALB/c samples indicate the activity of signaling pathways in M. gastrocnemius $(B$ and $D)$ and liver $(C)$ tissues. Bar diagrams show the quantifications of the results. Data are reported as means $\pm \mathrm{SE} ; n=3$ in each group. $* P<0.05 ; * * P<0.01$.

highest SUV mean, which was followed by the congenic wild-type and BALB/c groups.

\section{DISCUSSION}

Myostatin is a TGF $\beta$ family member that is expressed and secreted predominantly by skeletal muscle. The function of myostatin appears to be conserved across species, since mutations in the myostatin gene induce bigger muscles in human, mice, cattle, dogs, and sheep $(10,20,37,38,42)$. The Compact mice arose during a long-term selection program to reach the maximal hypermuscularity (7). The major gene responsible for the hypermuscular phenotype was mapped on chromosome 1 (49), and after the discovery of myostatin gene (31) the $M s t n(C m p t-d l 1 A b c)$ deletion in the propeptide region of the mouse myostatin was identified as the causative mutation responsible for the Compact phenotype (47). Due to selection for hypermuscularity, the Compact line, in addition to achieving homozygosity for the $\mathrm{Mstn}(\mathrm{Cmpt}-\mathrm{dll} \mathrm{Abc})$ mutation, also accumulated modifier alleles that were involved in the full expression of the phenotype $(47,48)$. Markers on several chromosomes (chromosomes 1, 3, 5, 7, 11, 16, and X) showed linkage with the putative modifiers, and the strongest associa- 
A
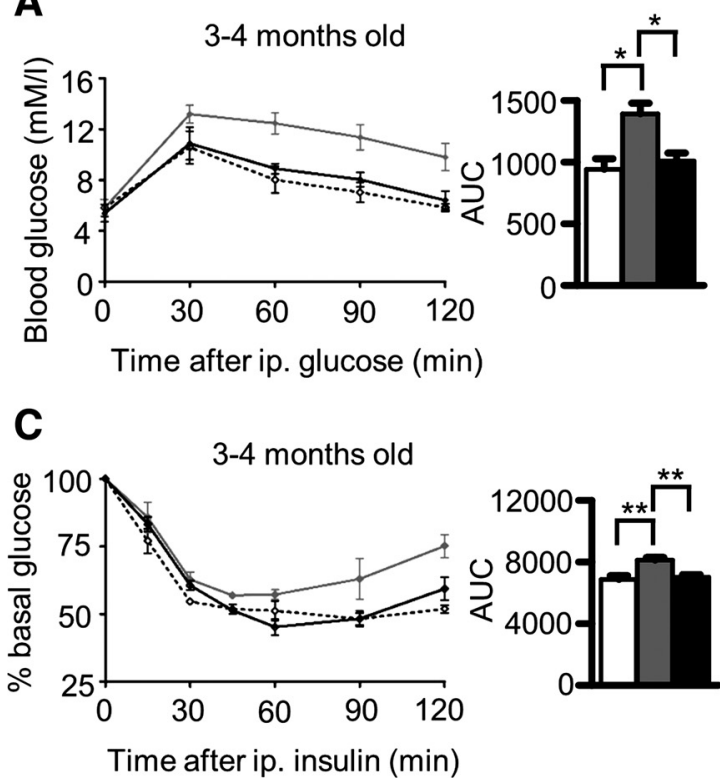

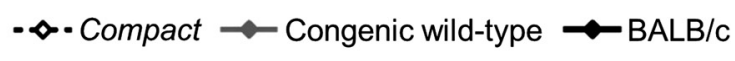

B

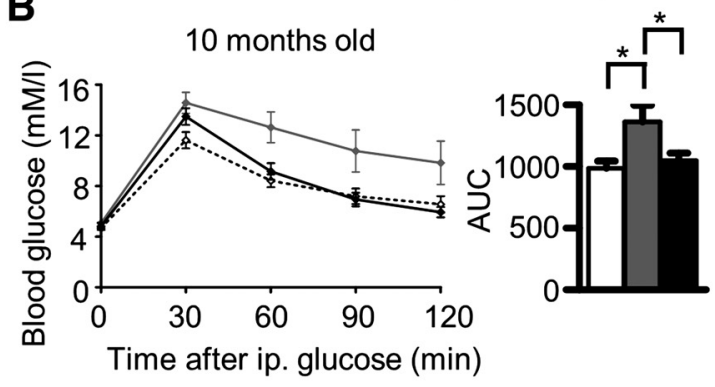

D

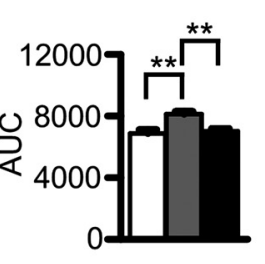

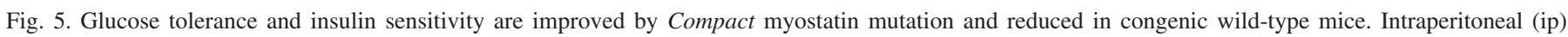

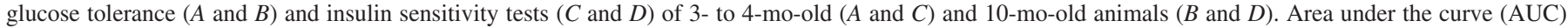

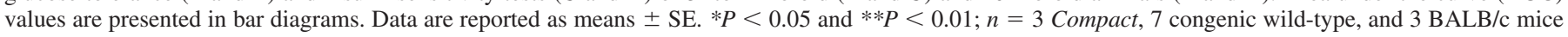

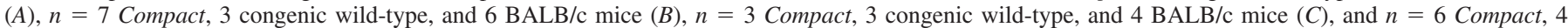
congenic wild-type, and $4 \mathrm{BALB} / \mathrm{c}$ mice $(D)$.

tion was found for markers on chromosomes 16 and $X(47,48)$. Myogenin is a candidate on chromosome 1 (48), which has been proposed as a downstream target of myostatin (23). Candidates localized on chromosome 7 are MyoD1, a key regulator of myogenesis, and Pcsk6 (protein convertase subtilisin/kexin type 6), which is involved in proteolytic processing of TGF $\beta$ members. Further candidates are chordin on chromosome 16, which binds BMPs and sequesters them in a latent complex, and androgen receptor on chromosome X (47).

Moreover, little is known about the detailed phenotype of Compact mice, and the molecular consequences of Compact mutation are completely unclear. We and others have reported

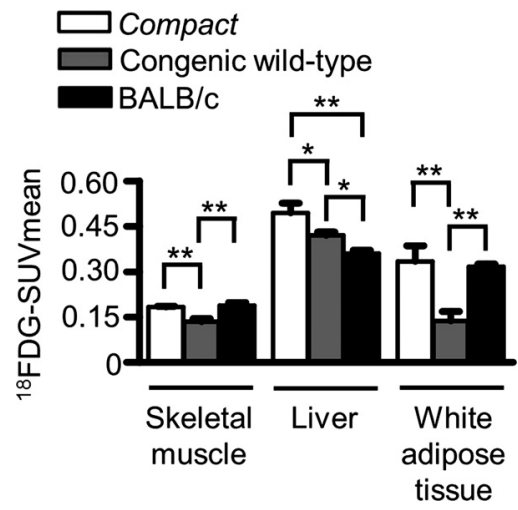

Fig. 6. Compact mutation increases ${ }^{18} \mathrm{FDG}$ (2-deoxy-2- $\left[{ }^{18} \mathrm{~F}\right]$ fluoro-D-glucose) uptake. Quantitative analysis of ${ }^{18} \mathrm{FDG}$ uptake of selected tissues in Compact, congenic, and BALB/c mice $50 \mathrm{~min}$ after tracer injection. Data are presented as means $\pm \mathrm{SE} ; n=3$ Compact, 4 congenic wild-type, and $4 \mathrm{BALB} / \mathrm{c}$ mice (skeletal muscle, liver); $n=4$ in each group (white adipose tissue). $* P<0.05$; $* * P<0.01$. earlier that the Compact mice are hypermuscular $(3,22,35)$, and cellularity of the Compact skeletal muscles shows increased ratio of glycolytic fibers in rectus femoris, longissimus dorsi (35), and tibialis anterior (3, 22) muscles. Decreased specific force (1) and reduced calcium release from sarcoplasmic reticulum (5) were reported in Compact muscles.

To separately study the effect of Compact myostatin mutation and Compact genetic background, we generated a congenic wild-type strain carrying wild-type myostatin in the Compact genetic background. We introgressed the wild-type myostatin gene of BALB/c to Compact mice to generate the congenic wild-type line, and we used BALB/c mice as a wild-type control for the reasons listed by Baán et al. (3). Briefly, this inbred line was used for mapping the Compact myostatin mutation and the modifier genes (42), and their muscle characteristics are similar to those of C57BL/6 mice. We have found that both the Compact myostatin mutation and the Compact genetic background account for determination of skeletal muscle size. The Compact mutation resulted in a disproportionate increase in skeletal muscle mass, leading to increased muscle/body weight ratios and decreased internal organ/body weight ratios. The Compact mice are weighted and hypermuscular compared with both congenic wild-type and $\mathrm{BALB} / \mathrm{c}$ animals. Interestingly, the normalized muscle weights of congenic wild-type animals are the smallest despite their increased absolute muscle weights compared with BALB/c mice; therefore, other organs should be involved in accretion of body weight of congenic wild-type animals.

Beyond the regulation of muscularity, myostatin was shown earlier to influence the size of internal organs. Knocking out of myostatin resulted in decreased weight of fat, liver, and kidney 
as proportional to body weight $(18,19,26,32)$. The absolute weight of heart increased; however, heart weight/body weight ratio did not change $(19,26)$. Bünger et al. (8) introgressed the Compact mutation into a mouse line with extreme growth (DUHi). The Compact mutation in the DUHi background decreased the absolute size of liver, heart, and kidney (8); in contrast, based on our study, the Compact mutation did not affect them in the Compact background, indicating the importance of genetic background in the manifestation of the phenotype. However, the Compact mutation resulted in hypermuscularity in both the DUHi (8) and Compact backgrounds as well. Similarly to myostatin knockouts, the liver/body weight ratio set point is reduced in Compacts. By testing the enzymatic functions of the liver, despite the smaller liver weight/ body weight ratio of Compacts, the hepatic gluconeogenesis was comparable with congenic wild-type animals following exogenous pyruvate. The tissue ALT activity of the liver was reduced by Compact myostatin mutation similarly to knocking out of myostatin (18).

Myostatin was reported to affect glucose metabolism; however, the fed and fasting glucose levels of myostatin knockout male mice were not different from controls (32). Similarly to these observations, fasting blood glucose levels of Compact mice did not change compared with age-matched congenic wild-type and BALB/c animals. Furthermore, myostatin-null mice have reduced body fat beyond the increased muscle mass and exhibit increased insulin sensitivity $(14,55)$. Here, we showed that Compact mutation increased muscularity and decreased adiposity similarly to myostatin knockout mice, whereas the genetic background had the opposite effect, increasing adiposity and decreasing skeletal muscle mass/body weight ratios. As a consequence of these alterations in body composition, the Compact mutation improved whole body glucose tolerance and insulin sensitivity, whereas the genetic background decreased them. The Compact mutation increased the ${ }^{18} \mathrm{FDG}$ radiotracer uptake into all investigated organs (white adipose tissue, skeletal muscle, and liver). The phosphorylation of AS160 was shown to regulate the translocation of GLUT4 to the plasma membrane $(21,36)$. However, the Compact mutation did not influence the amount of GLUT4 in skeletal muscle, but the increased p-AS160 level in Compact animals might contribute to the increased glucose uptake by enhancing the GLUT4 translocation to the plasma membrane.

Besides the regulation of insulin sensitivity and glucose tolerance, the Compact genetic background has a role in the regulation of tissue glycogen content. It has opposite impacts on skeletal muscle and liver by decreasing the glycogen level/ tissue weight ratio in muscle and increasing it in the liver. These alterations can be at least partially the consequences of decreased glucose uptake into muscle and increased glucose uptake into liver. Consistent with these results, reduced muscle glycogen was reported in type 2 diabetes mellitus (15). The Compact mutation itself did not affect the glycogen level/tissue weight ratio in either muscle or liver tissue; however, myostatin treatment was found to reduce the glycogen content of C2C12 myoblasts (9).

Age was shown to affect glucose metabolism; however, no difference was reported in glucose clearance rate comparing 6and 12-mo-old BALB/c mice (33). Consistent with these results, the glucose tolerance of $\mathrm{BALB} / \mathrm{c}$ animals did not change with age (3-4 vs. 10 mo old) in our study. Interestingly, aging reduced glucose tolerance of Compact mice without any significant alteration in sensitivity for exogenous insulin.

Although the Compact mutation was identified in 1998 (42), its precise molecular effects have not yet been published. The Compact mutation is a nonframeshift deletion in the propeptide; therefore, it raised the possibility that the mature myostatin is present in Compact mice. Here, we have shown that the Compact mutation allows the formation of mature myostatin; however, the amount of myostatin was lower in Compact skeletal muscle, in accordance with increased muscle mass. Most of the naturally occurring myostatin mutations led to the development of an early STOP codon; however, some mutations were shown to be associated with altered proteolysis of promyostatin $(4,43)$ permitting myostatin formation.

The specific functions of the propeptides of TGF superfamily members are largely unknown; however, they can play a role in targeting and inactivation of the biological active $\mathrm{COOH}$-terminal part, and they have an impact on binding properties to extracellular components. All of these functions of myostatin propeptide can be disturbed by Compact mutation. It was reported that BMP-7 propeptide binds fibrillin-1 (39), BMP-5 propeptide binds fibrillin-1 and -2 (39), and the interaction between myostatin propeptide and perlecan was identified (40). The interaction of propeptide and myostatin is relevant in vivo, with a majority $(>70 \%)$ of myostatin in serum bound to its propeptide (16). The amino acid sequence of GDF-11 (growth/differentiation factor-11) is 90\% homologous to myostatin in the carboxy-terminal mature region of the protein, and like myostatin, GDF-11 can signal through ActRIIB (30), and GDF-11 administration leads to activation of Smad2 signaling $(12,34)$. Myostatin propeptide may bind and inhibit GDF-11 as well as myostatin (30); therefore, the mutant propeptide of Compact mice might disturb not only myostatin but, e.g., GDF-11 signaling as well.

Despite the low level of myostatin protein in Compact skeletal muscle, the level of phospo-Smad2 was the highest when the genotypes were compared, suggesting the potential role of other TGF $\beta$ members (e.g., GDF-11) or HGF (hepatocyte growth factor) in Smad2 activation. HGF is a regulator of satellite cells (44) and transmits signals through Smad2/3. The BMP-mediated Smad1/5/8 signaling is an important regulator of skeletal muscle mass $(37,52)$, hepatocyte proliferation, and liver regeneration and function $(11,41)$. Comparing the genotypes, phospho-Smad1/5/8 levels show the same pattern as tissue weight/body weight ratios in both skeletal muscle and liver, suggesting the impact of Smad1/5/8 signaling on regulation of skeletal muscle and liver size. The BMP inhibitor chordin was proposed as a modifier gene in Compact mice (48) that can influence the activity of BMP pathway by binding and modulating the effect of BMPs (54). Interestingly, the elevated phospho-Smad1/5/8 levels can derive not just from BMPs, since TGF $\beta$ was reported to stimulate the phosphorylation of Smad1/5 through a noncanonical mechanism (27).

The Compact mice represent a complex system consisting of a natural mutation in the propeptide of promyostatin and additional modifier genes. The Compact mice show several similarities compared with myostatin knockout animals; however, numerous alterations exist due to the redundant function of propeptide and the presence of the specific Compact genetic background. Myostatin propeptide may bind and inhibit GDF-11 as well (30); therefore, the effect of Compact propep- 
tide cannot be restricted to myostatin signaling. Our analysis has shown that the modifier genes of the genetic background can strengthen the effect of Compact myostatin mutation, or they can compensate for each other. Further analysis of the biological effect of Compact mutation and the identification of modifier genes may provide a route to additional upstream and downstream factors involved in the regulation of skeletal muscle size and metabolism.

\section{ACKNOWLEDGMENTS}

We thank Zita Makrane Felho and Laszlone Csontos for excellent technical assistance. We thank Jozsef Mihaly for providing horseradish peroxidaseconjugated anti-rat secondary antibody.

\section{GRANTS}

This research was supported by the European Union and the State of Hungary and cofinanced by the European Social Fund in the framework of the TÁMOP 4.2.4. A/2-11-1-2012-0001 "National Excellence Program" (to A. Keller-Pinter) and GINOP-2.3.2-15-2016-00006.

\section{DISCLOSURES}

No conflicts of interest, financial or otherwise, are declared by the authors.

\section{AUTHOR CONTRIBUTIONS}

T.K., G.T., K.S., and A.K.-P. performed experiments; T.K., G.T., and A.K.-P. analyzed data; T.K., G.T., J.A.B., and A.K.-P. interpreted results of experiments; T.K. and A.K.-P. prepared figures; T.K., G.T., L.M., I.G., H.R., F.D., and A.K.-P. drafted manuscript; T.K., F.D., L.D., and A.K.-P. edited and revised manuscript; T.K., G.T., K.S., J.A.B., G.M., L.M., I.G., H.R., F.D., L.D., and A.K.-P. approved final version of manuscript.

\section{REFERENCES}

1. Amthor H, Macharia R, Navarrete R, Schuelke M, Brown SC, Otto A, Voit T, Muntoni F, Vrbóva G, Partridge T, Zammit P, Bunger L, Patel K. Lack of myostatin results in excessive muscle growth but impaired force generation. Proc Natl Acad Sci USA 104: 1835-1840, 2007. doi:10.1073/pnas.0604893104.

2. Ayala JE, Samuel VT, Morton GJ, Obici S, Croniger CM, Shulman GI, Wasserman DH, McGuinness OP; NIH Mouse Metabolic Phenotyping Center Consortium. Standard operating procedures for describing and performing metabolic tests of glucose homeostasis in mice. Dis Model Mech 3: 525-534, 2010. doi:10.1242/dmm.006239.

3. Baán JA, Kocsis T, Keller-Pintér A, Müller G, Zádor E, Dux L, Mendler L. The compact mutation of myostatin causes a glycolytic shift in the phenotype of fast skeletal muscles. J Histochem Cytochem 61: 889-900, 2013. doi:10.1369/0022155413503661.

4. Berry C, Thomas M, Langley B, Sharma M, Kambadur R. Single cysteine to tyrosine transition inactivates the growth inhibitory function of Piedmontese myostatin. Am J Physiol Cell Physiol 283: C135-C141, 2002. doi:10.1152/ajpcell.00458.2001.

5. Bodnár D, Geyer N, Ruzsnavszky O, Oláh T, Hegyi B, Sztretye M, Fodor J, Dienes B, Balogh Á, Papp Z, Szabó L, Müller G, Csernoch L, Szentesi P. Hypermuscular mice with mutation in the myostatin gene display altered calcium signalling. $J$ Physiol 592: 1353-1365, 2014. doi:10.1113/jphysiol.2013.261958.

6. Brandt C, Hansen RH, Hansen JB, Olsen CH, Galle P, Mandrup-Poulsen T, Gehl J, Pedersen BK, Hojman P. Over-expression of Follistatin-like 3 attenuates fat accumulation and improves insulin sensitivity in mice. Metabolism 64: 283-295, 2015. doi:10.1016/j.metabol.2014.10.007.

7. Bünger L, Laidlaw A, Bulfield G, Eisen EJ, Medrano JF, Bradford GE, Pirchner F, Renne U, Schlote W, Hill WG. Inbred lines of mice derived from long-term growth selected lines: unique resources for mapping growth genes. Mamm Genome 12: 678-686, 2001. doi:10.1007/ s00335001-3018-6.

8. Bünger L, Ott G, Varga L, Schlote W, Rehfeldt C, Renne U, Williams JL, Hill WG. Marker-assisted introgression of the Compact mutant myostatin allele MstnCmpt-dl1 Abc into a mouse line with extreme growth effects on body composition and muscularity. Genet Res 84: 161-173, 2004. doi:10.1017/S0016672304007165.
9. Chen Y, Ye J, Cao L, Zhang Y, Xia W, Zhu D. Myostatin regulates glucose metabolism via the AMP-activated protein kinase pathway in skeletal muscle cells. Int J Biochem Cell Biol 42: 2072-2081, 2010. doi:10.1016/j.biocel.2010.09.017.

10. Clop A, Marcq F, Takeda H, Pirottin D, Tordoir X, Bibé B, Bouix J, Caiment F, Elsen JM, Eychenne F, Larzul C, Laville E, Meish F, Milenkovic D, Tobin J, Charlier C, Georges M. A mutation creating a potential illegitimate microRNA target site in the myostatin gene affects muscularity in sheep. Nat Genet 38: 813-818, 2006. doi:10.1038/ng1810.

11. Do N, Zhao R, Ray K, Ho K, Dib M, Ren X, Kuzontkoski P, Terwilliger E, Karp SJ. BMP4 is a novel paracrine inhibitor of liver regeneration. Am J Physiol Gastrointest Liver Physiol 303: G1220G1227, 2012. doi:10.1152/ajpgi.00105.2012.

12. Egerman MA, Cadena SM, Gilbert JA, Meyer A, Nelson HN, Swalley SE, Mallozzi C, Jacobi C, Jennings LL, Clay I, Laurent G, Ma S, Brachat S, Lach-Trifilieff E, Shavlakadze T, Trendelenburg AU, Brack AS, Glass DJ. GDF11 Increases with Age and Inhibits Skeletal Muscle Regeneration. Cell Metab 22: 164-174, 2015. doi:10.1016/j.cmet. 2015.05.010

13. Guo T, Bond ND, Jou W, Gavrilova O, Portas J, McPherron AC. Myostatin inhibition prevents diabetes and hyperphagia in a mouse model of lipodystrophy. Diabetes 61: 2414-2423, 2012. doi:10.2337/ db11-0915.

14. Guo T, Jou W, Chanturiya T, Portas J, Gavrilova O, McPherron AC. Myostatin inhibition in muscle, but not adipose tissue, decreases fat mass and improves insulin sensitivity. PLoS One 4: e4937, 2009. doi:10.1371/ journal.pone.0004937.

15. He J, Kelley DE. Muscle glycogen content in type 2 diabetes mellitus. Am J Physiol Endocrinol Metab 287: E1002-E1007, 2004. doi:10.1152/ ajpendo.00015.2004

16. Hill JJ, Davies MV, Pearson AA, Wang JH, Hewick RM, Wolfman NM, Qiu Y. The myostatin propeptide and the follistatin-related gene are inhibitory binding proteins of myostatin in normal serum. J Biol Chem 277: 40735-40741, 2002. doi:10.1074/jbc.M206379200.

17. Hittel DS, Berggren JR, Shearer J, Boyle K, Houmard JA. Increased secretion and expression of myostatin in skeletal muscle from extremely obese women. Diabetes 58: 30-38, 2009. doi:10.2337/db08-0943.

18. Huang J, Glauber M, Qiu Z, Gazit V, Dietzen DJ, Rudnick DA. The influence of skeletal muscle on the regulation of liver:body mass and liver regeneration. Am J Pathol 180: 575-582, 2012. doi:10.1016/j.ajpath.2011. 10.032 .

19. Jackson MF, Luong D, Vang DD, Garikipati DK, Stanton JB, Nelson OL, Rodgers BD. The aging myostatin null phenotype: reduced adiposity, cardiac hypertrophy, enhanced cardiac stress response, and sexual dimorphism. J Endocrinol 213: 263-275, 2012. doi:10.1530/JOE-11-0455.

20. Kambadur R, Sharma M, Smith TP, Bass JJ. Mutations in myostatin (GDF8) in double-muscled Belgian Blue and Piedmontese cattle. Genome Res 7: 910-916, 1997.

21. Klip A, Sun Y, Chiu TT, Foley KP. Signal transduction meets vesicle traffic: the software and hardware of GLUT4 translocation. Am J Physiol Cell Physiol 306: C879-C886, 2014. doi:10.1152/ajpcell.00069.2014.

22. Kocsis T, Baán J, Müller G, Mendler L, Dux L, Keller-Pintér A. Skeletal muscle cellularity and glycogen distribution in the hypermuscular Compact mice. Eur J Histochem 58: 2353, 2014. doi:10.4081/ejh. 2014.2353.

23. Langley B, Thomas M, Bishop A, Sharma M, Gilmour S, Kambadur R. Myostatin inhibits myoblast differentiation by down-regulating MyoD expression. J Biol Chem 277: 49831-49840, 2002. doi:10.1074/jbc. M204291200.

24. Lee SJ, McPherron AC. Regulation of myostatin activity and muscle growth. Proc Natl Acad Sci USA 98: 9306-9311, 2001. doi:10.1073/pnas. 151270098.

25. Li Z, Zhao B, Kim YS, Hu CY, Yang J. Administration of a mutated myostatin propeptide to neonatal mice significantly enhances skeletal muscle growth. Mol Reprod Dev 77: 76-82, 2010. doi:10.1002/mrd. 21111.

26. Lin J, Arnold HB, Della-Fera MA, Azain MJ, Hartzell DL, Baile CA. Myostatin knockout in mice increases myogenesis and decreases adipogenesis. Biochem Biophys Res Commun 291: 701-706, 2002. doi:10.1006/ bbrc. 2002.6500 .

27. Liu IM, Schilling SH, Knouse KA, Choy L, Derynck R, Wang XF. TGFbeta-stimulated Smad1/5 phosphorylation requires the ALK5 L45 loop and mediates the pro-migratory TGFbeta switch. EMBO J 28: 88-98, 2009. doi:10.1038/emboj.2008.266. 
28. Mathew D, Zhou P, Pywell CM, van der Veen DR, Shao J, Xi Y, Bonar NA, Hummel AD, Chapman S, Leevy WM, Duffield GE. Ablation of the ID2 gene results in altered circadian feeding behavior, and sex-specific enhancement of insulin sensitivity and elevated glucose uptake in skeletal muscle and brown adipose tissue. PLoS One 8: e73064, 2013. doi:10.1371/journal.pone.0073064.

29. McCroskery S, Thomas M, Maxwell L, Sharma M, Kambadur R. Myostatin negatively regulates satellite cell activation and self-renewal. $J$ Cell Biol 162: 1135-1147, 2003. doi:10.1083/jcb.200207056.

30. McPherron AC. Metabolic functions of myostatin and GDF11. Immunol Endocr Metab Agents Med Chem 10: 217-231, 2010. doi:10.2174/ 187152210793663810.

31. McPherron AC, Lawler AM, Lee SJ. Regulation of skeletal muscle mass in mice by a new TGF-beta superfamily member. Nature 387: 83-90, 1997. doi:10.1038/387083a0.

32. McPherron AC, Lee SJ. Suppression of body fat accumulation in myostatin-deficient mice. J Clin Invest 109: 595-601, 2002. doi:10.1172/ JCI0213562.

33. Nankervis SA, Mitchell JM, Charchar FJ, McGlynn MA, Lewandowski PA. Consumption of a low glycaemic index diet in late life extends lifespan of Balb/c mice with differential effects on DNA damage. Longev Healthspan 2: 4, 2013. doi:10.1186/2046-2395-2-4.

34. Poggioli T, Vujic A, Yang P, Macias-Trevino C, Uygur A, Loffredo FS, Pancoast JR, Cho M, Goldstein J, Tandias RM, Gonzalez E, Walker RG, Thompson TB, Wagers AJ, Fong YW, Lee RT. Circulating growth differentiation factor 11/8 levels decline with Age. Circ Res 118: 29-37, 2016. doi:10.1161/CIRCRESAHA.115.307521.

35. Rehfeldt C, Ott G, Gerrard DE, Varga L, Schlote W, Williams JL, Renne U, Bünger L. Effects of the compact mutant myostatin allele Mstn (Cmpt-dl1 Abc) introgressed into a high growth mouse line on skeletal muscle cellularity. J Muscle Res Cell Motil 26: 103-112, 2005. doi:10. 1007/s10974-005-1099-7.

36. Sakamoto K, Holman GD. Emerging role for AS160/TBC1D4 and TBC1D1 in the regulation of GLUT4 traffic. Am J Physiol Endocrinol Metab 295: E29-E37, 2008. doi:10.1152/ajpendo.90331.2008.

37. Sartori R, Schirwis E, Blaauw B, Bortolanza S, Zhao J, Enzo E, Stantzou A, Mouisel E, Toniolo L, Ferry A, Stricker S, Goldberg AL, Dupont S, Piccolo S, Amthor H, Sandri M. BMP signaling controls muscle mass. Nat Genet 45: 1309-1318, 2013. doi:10.1038/ng.2772.

38. Schuelke M, Wagner KR, Stolz LE, Hübner C, Riebel T, Kömen W, Braun T, Tobin JF, Lee SJ. Myostatin mutation associated with gross muscle hypertrophy in a child. $N$ Engl J Med 350: 2682-2688, 2004. doi:10.1056/NEJMoa040933.

39. Sengle G, Charbonneau NL, Ono RN, Sasaki T, Alvarez J, Keene DR, Bächinger HP, Sakai LY. Targeting of bone morphogenetic protein growth factor complexes to fibrillin. J Biol Chem 283: 13874-13888, 2008. doi:10.1074/jbc.M707820200.

40. Sengle G, Ono RN, Sasaki T, Sakai LY. Prodomains of transforming growth factor $\beta$ (TGF $\beta$ ) superfamily members specify different functions: extracellular matrix interactions and growth factor bioavailability. $J$ Biol Chem 286: 5087-5099, 2011. doi:10.1074/jbc.M110.188615.

41. Sugimoto H, Yang C, LeBleu VS, Soubasakos MA, Giraldo M, Zeisberg M, Kalluri R. BMP-7 functions as a novel hormone to facilitate liver regeneration. FASEB J 21: 256-264, 2006. doi:10.1096/fj.06-6837com.

42. Szabó G, Dallmann G, Müller G, Patthy L, Soller M, Varga L. A deletion in the myostatin gene causes the compact (Cmpt) hypermuscular mutation in mice. Mamm Genome 9: 671-672, 1998. doi:10.1007/ s003359900843.
43. Szláma G, Trexler M, Buday L, Patthy L. K153R polymorphism in myostatin gene increases the rate of promyostatin activation by furin. FEBS Lett 589: 295-301, 2015. doi:10.1016/j.febslet.2014.12.011.

44. Tatsumi R, Anderson JE, Nevoret CJ, Halevy O, Allen RE. HGF/SF is present in normal adult skeletal muscle and is capable of activating satellite cells. Dev Biol 194: 114-128, 1998. doi:10.1006/dbio.1997. 8803.

45. Thies RS, Chen T, Davies MV, Tomkinson KN, Pearson AA, Shakey QA, Wolfman NM. GDF-8 propeptide binds to GDF-8 and antagonizes biological activity by inhibiting GDF-8 receptor binding. Growth Factors 18: 251-259, 2001. doi:10.3109/08977190109029114.

46. Thomas M, Langley B, Berry C, Sharma M, Kirk S, Bass J, Kambadur R. Myostatin, a negative regulator of muscle growth, functions by inhibiting myoblast proliferation. J Biol Chem 275: 40235-40243, 2000. doi:10.1074/jbc.M004356200.

47. Varga L, Müller G, Szabó G, Pinke O, Korom E, Kovács B, Patthy L, Soller M. Mapping modifiers affecting muscularity of the myostatin mutant (Mstn(Cmpt-d11Abc)) compact mouse. Genetics 165: 257-267, 2003.

48. Varga L, Pinke O, Müller G, Kovács B, Korom E, Szabó G, Soller M. Mapping a syntenic modifier on mouse chromosome 1 influencing the expressivity of the compact phenotype in the myostatin mutant (MstnCmpt-dl1 Abc) compact mouse. Genetics 169: 489-493, 2005. doi:10.1534/ genetics.104.034033

49. Varga L, Szabó G, Darvasi A, Müller G, Sass M, Soller M. Inheritance and mapping of Compact (Cmpt), a new mutation causing hypermuscularity in mice. Genetics 147: 755-764, 1997.

50. Weniger JH, Horst P, Steinhauf D, Major F, Wolf M, Tawfik ES. Model experiments on selection for endurance and its relation to growth. Part I. Introduction, methods and preliminary investigations on the basic population. J Anim Breed Genet 91: 265-270, 1974.

51. Williams NG, Interlichia JP, Jackson MF, Hwang D, Cohen P, Rodgers BD. Endocrine actions of myostatin: systemic regulation of the IGF and IGF binding protein axis. Endocrinology 152: 172-180, 2011. doi:10. 1210/en.2010-0488.

52. Winbanks CE, Chen JL, Qian H, Liu Y, Bernardo BC, Beyer C, Watt KI, Thomson RE, Connor T, Turner BJ, McMullen JR, Larsson L, McGee SL, Harrison CA, Gregorevic P. The bone morphogenetic protein axis is a positive regulator of skeletal muscle mass. J Cell Biol 203: 345-357, 2013. doi:10.1083/jcb.201211134.

53. Yang W, Zhang Y, Li Y, Wu Z, Zhu D. Myostatin induces cyclin D1 degradation to cause cell cycle arrest through a phosphatidylinositol 3-kinase/AKT/GSK-3 beta pathway and is antagonized by insulin-like growth factor 1. J Biol Chem 282: 3799-3808, 2007. doi:10.1074/jbc. M610185200.

54. Zakin L, De Robertis EM. Extracellular regulation of BMP signaling. Curr Biol 20: R89-R92, 2010. doi:10.1016/j.cub.2009.11.021.

55. Zhang C, McFarlane C, Lokireddy S, Bonala S, Ge X, Masuda S, Gluckman PD, Sharma M, Kambadur R. Myostatin-deficient mice exhibit reduced insulin resistance through activating the AMP-activated protein kinase signalling pathway. Diabetologia 54: 1491-1501, 2011. doi:10.1007/s00125-011-2079-7.

56. Zhao B, Wall RJ, Yang J. Transgenic expression of myostatin propeptide prevents diet-induced obesity and insulin resistance. Biochem Biophys Res Commun 337: 248-255, 2005. doi:10.1016/j.bbrc.2005.09.044.

57. Zhu X, Topouzis S, Liang LF, Stotish RL. Myostatin signaling through Smad2, Smad3 and Smad4 is regulated by the inhibitory Smad7 by a negative feedback mechanism. Cytokine 26: 262-272, 2004. doi:10.1016/ j.cyto.2004.03.007. 